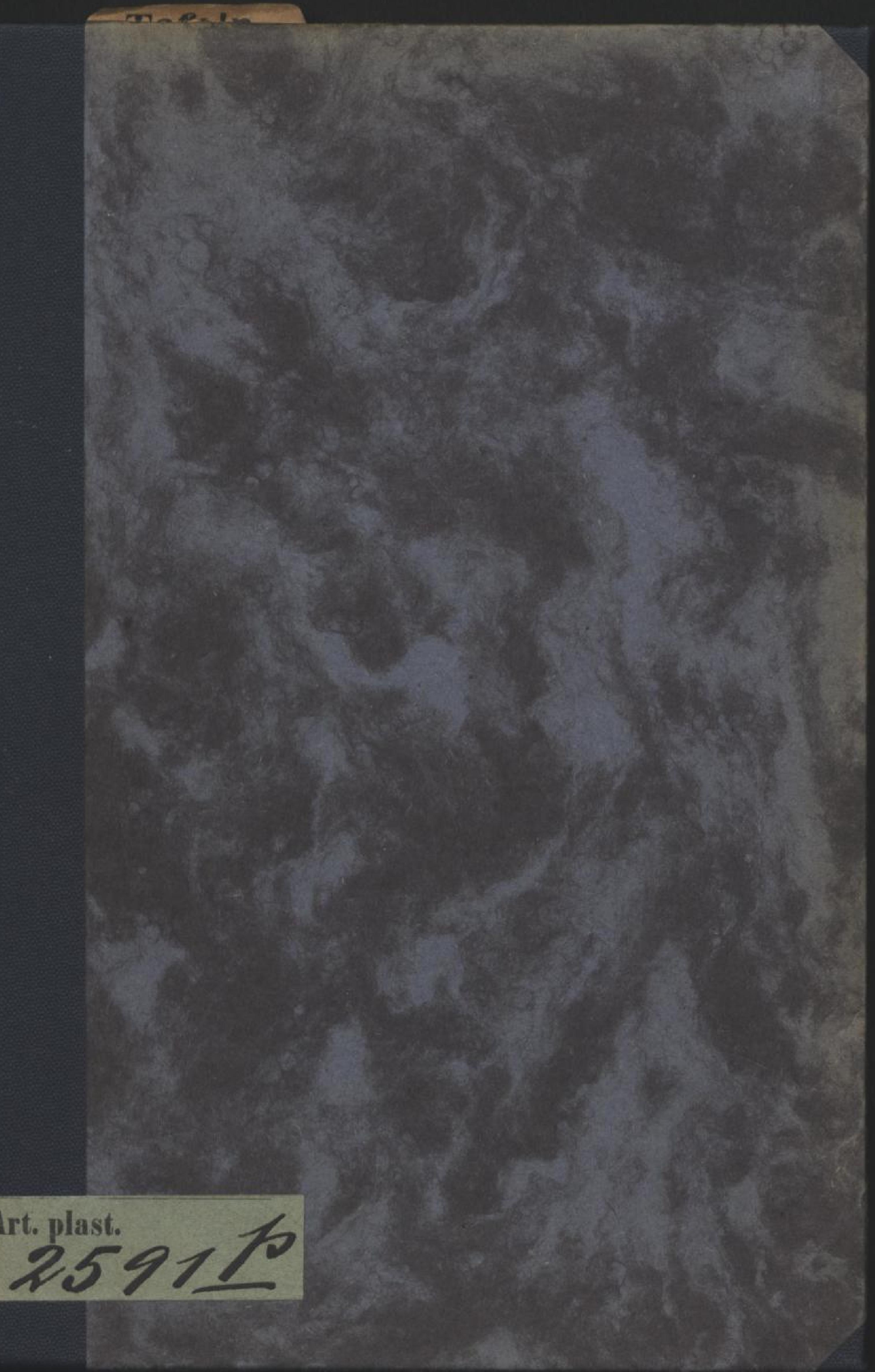

Art. plast.

gefördert von der

Deutschen Forschungsgemeinschaft $\mathrm{D}=\mathrm{C}$ 


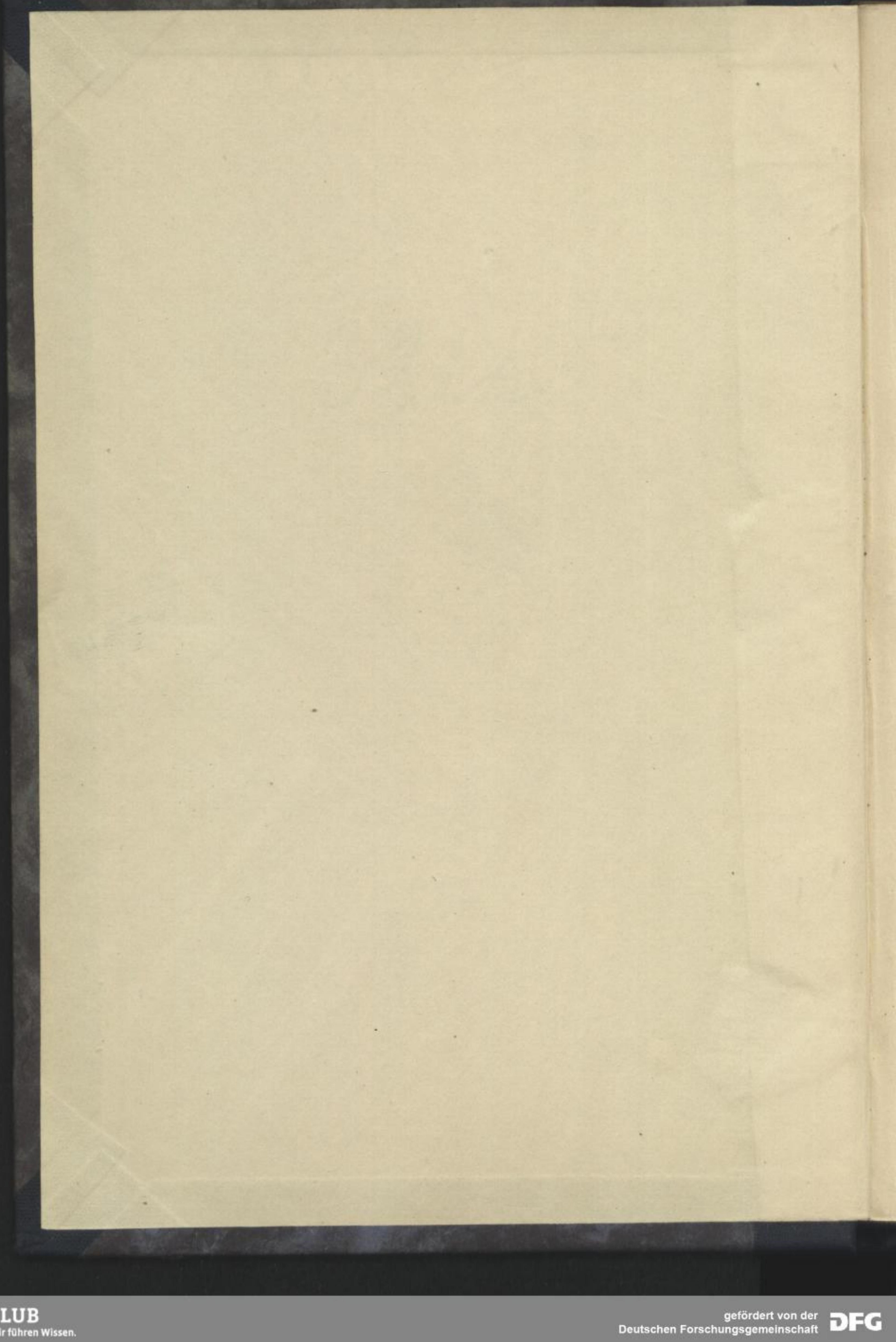




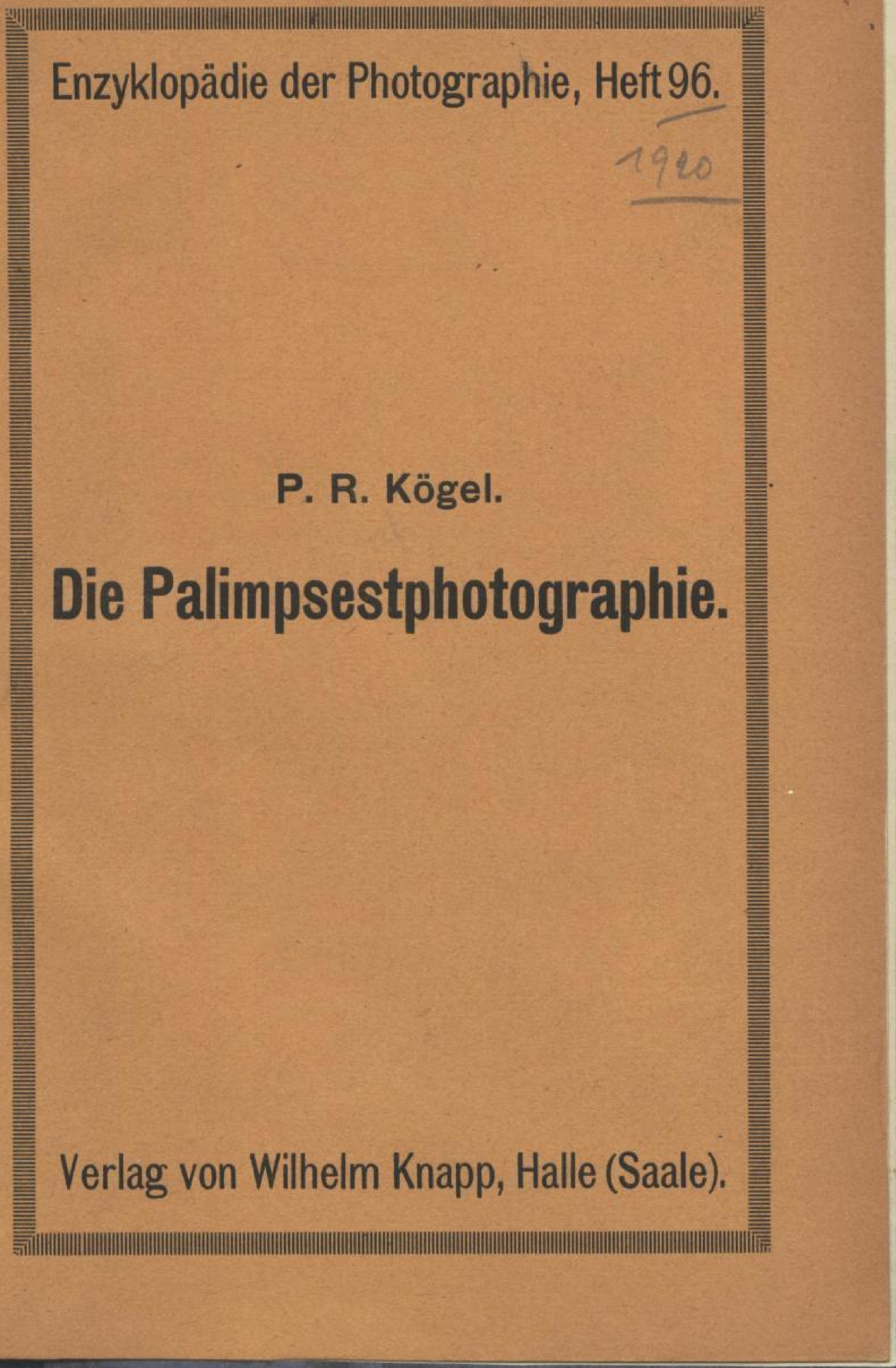




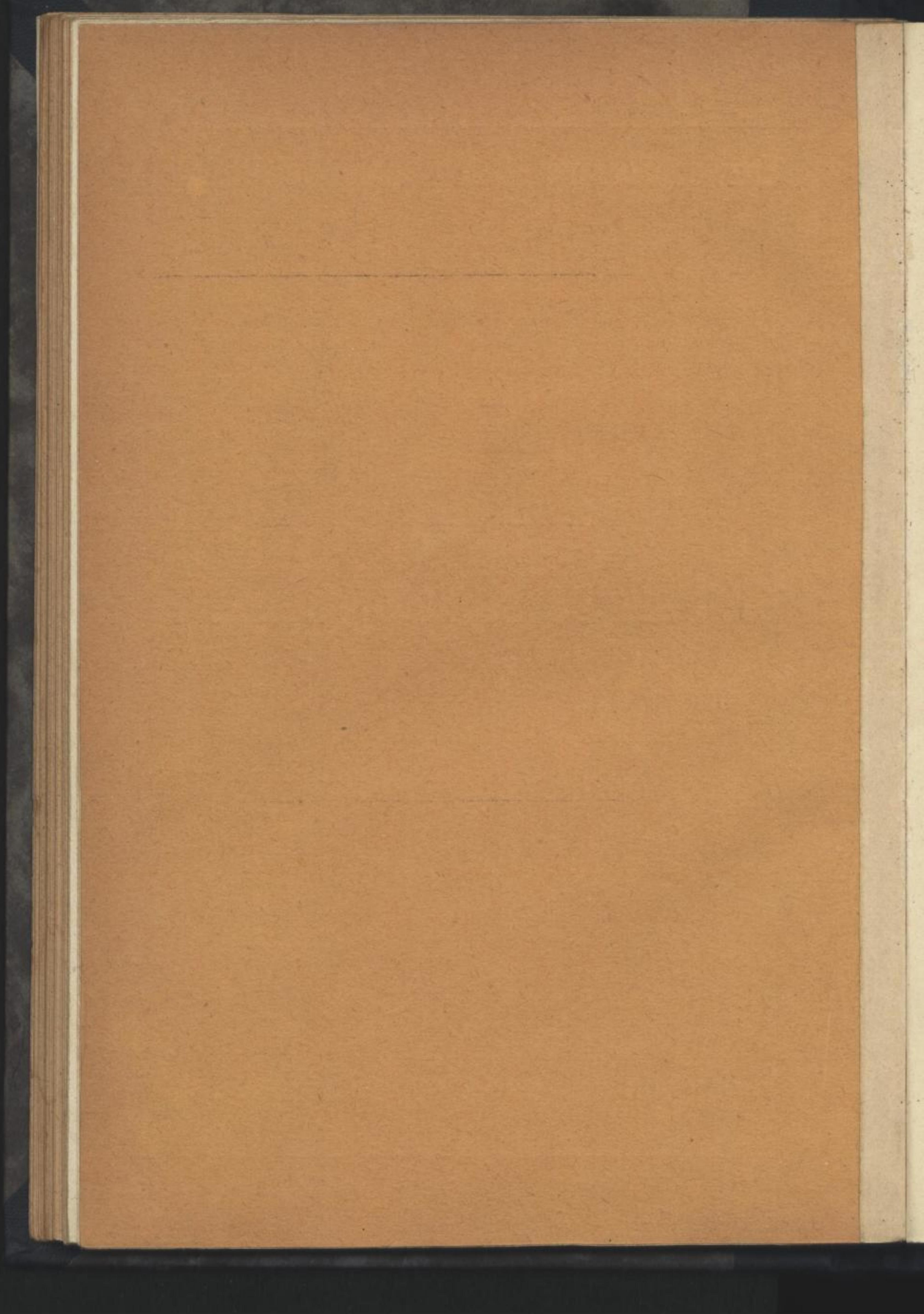

SLUB Wir fuhren Wissen. gefördert von der
Deutschen Forschungsgemeinschaft 
Enzyklopädie der Photographie, Heft 95.6

\section{Die Palimpsestphotographie} (Photographie der radierten Schriften)

in ihren wissenschaftlichen Grundlagen und praktischen Anwendungen

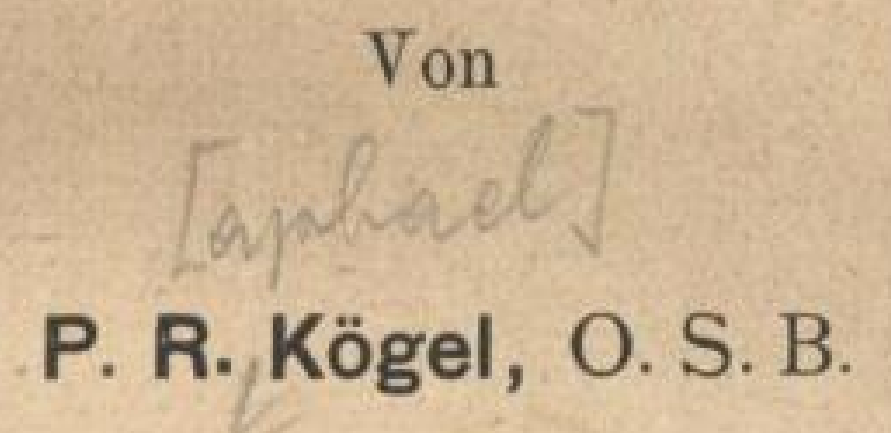

Mit 42 Abbildungen auf 8 Tafeln.

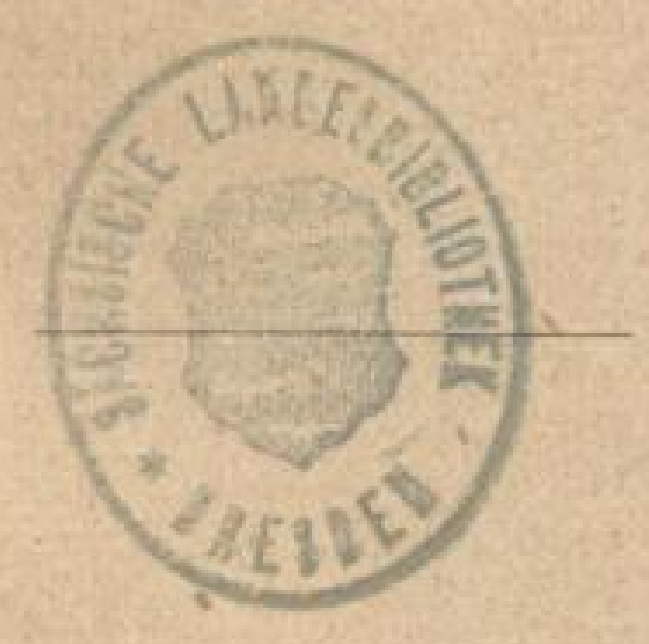

Halle (Saale).

Druck und Verlag von Wilhelm Knapp. 1920. 


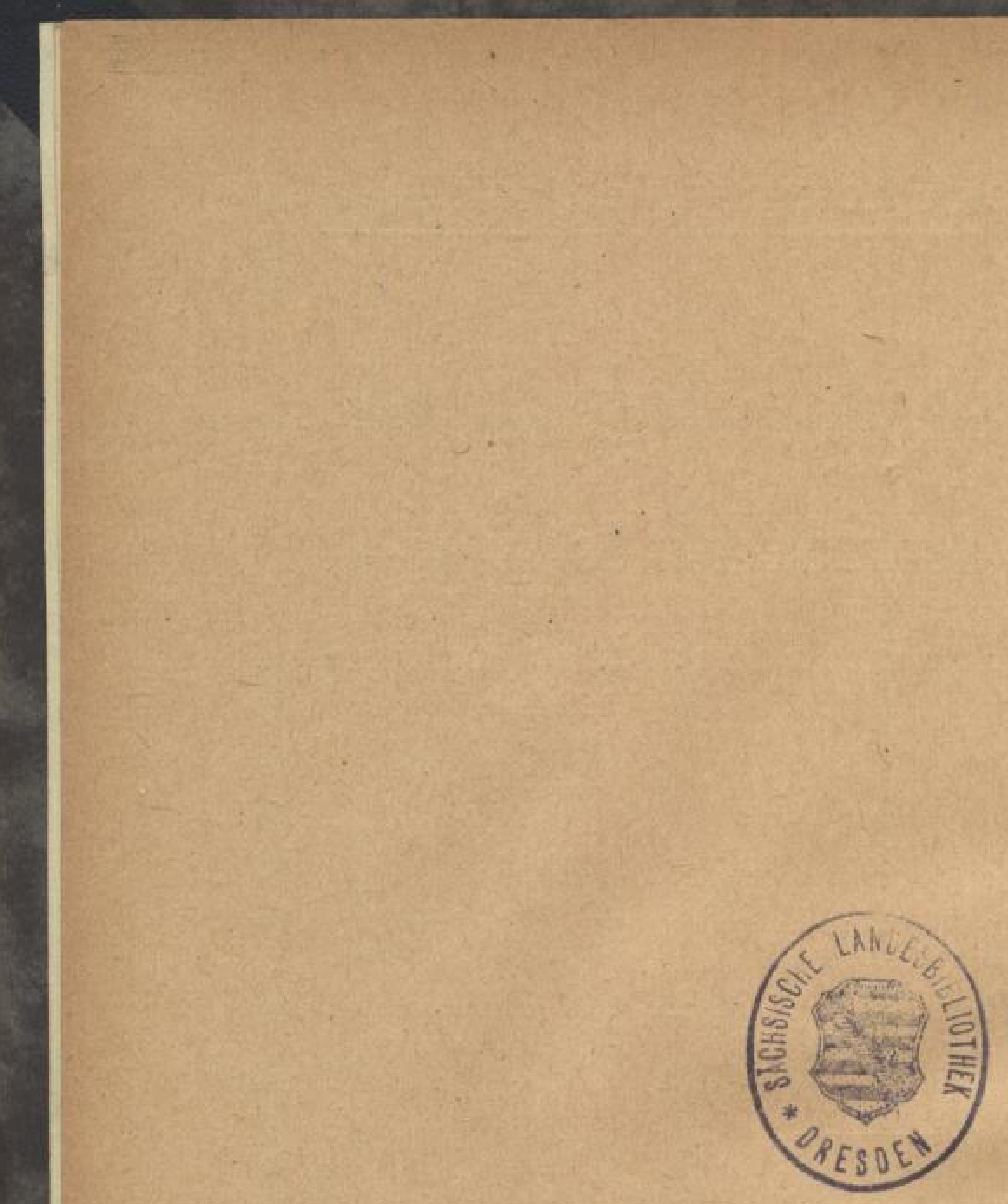


- Einem hochverdienten Förderer der Palimpsestkunde,

\section{Herrn Dr. 0. Gradenwitz,}

o. б. Professor des Rómischen Rechtes an der Universităt Heidelberg,

und dem Andenken seines Freundes und Mitarbeiters

\section{Herrn Dr. E. Pringsheim,}

weil. o.. . Professor der Theor. Physik an der Universital Breslau,

in Verehrung gewidmet vom

Verfasser. 


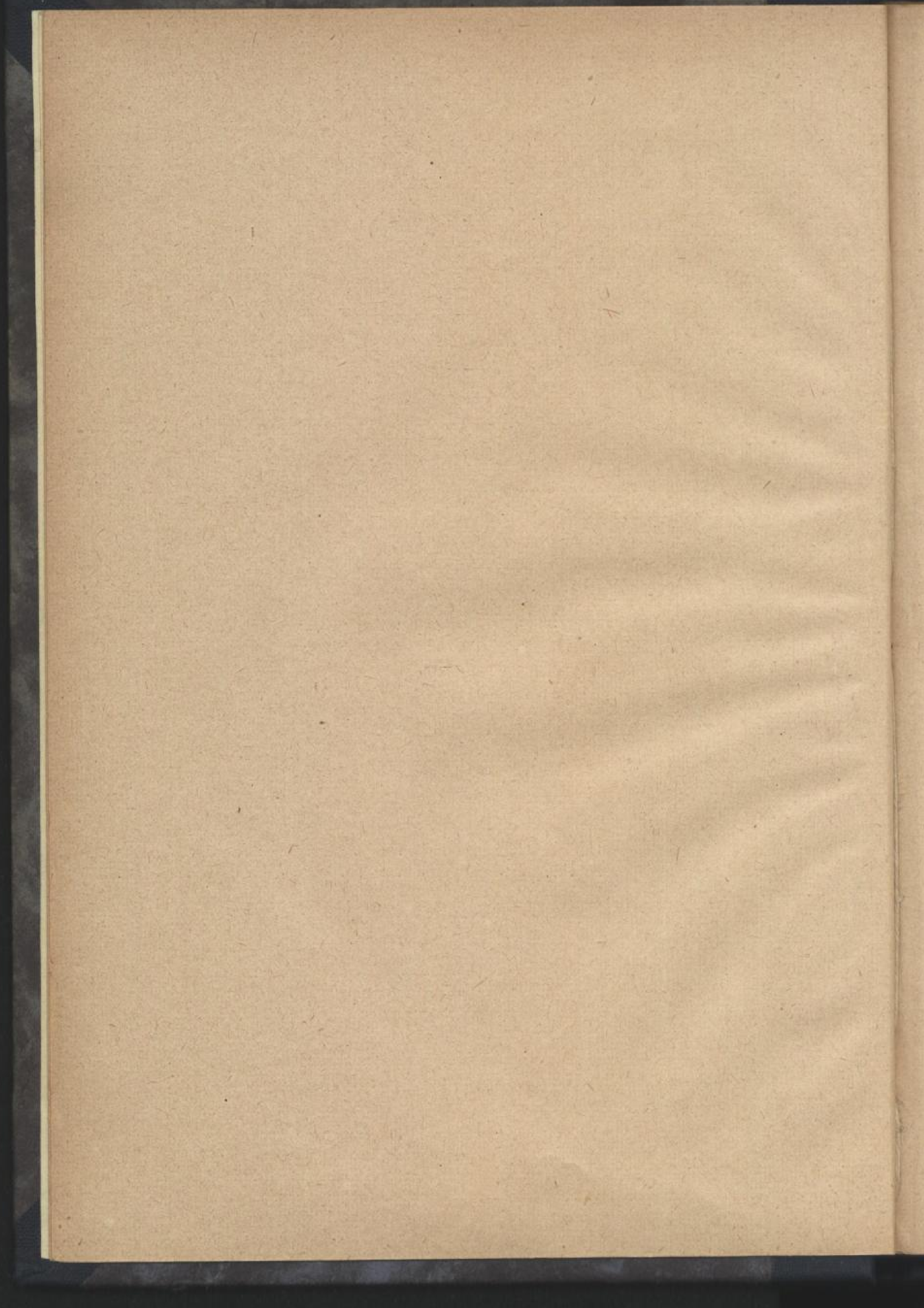

SLUB gefördert von der
Deutschen Forschungsgemeinschaft 
Ars photographica.

Expressa solis speculo

Nitens imago, quam bene

Frontis decus, vim luminum

Refert et oris gratiam!

0 mira virtus ingenii!

Novumque monstrum! imaginem

Naturae Apelles aemulus

Non pulchriorem pingueret.

Vom Sonnenspiegel hingehaucht

Erscheint ein glänzendes Bild, wie schön

Strahlt es die Stirne, das Augenlicht,

Des Mundes Anmut hold zurück!

O wunderbare Geistesmacht!

Ein neu Gebilde der Natur,

Wie selbst Apelles' Meisterhand

Es schöner nicht hervorgebracht.

Leo XIII. 1877.

Eder, Geschichte der Photographie, 1905, S. 297. 


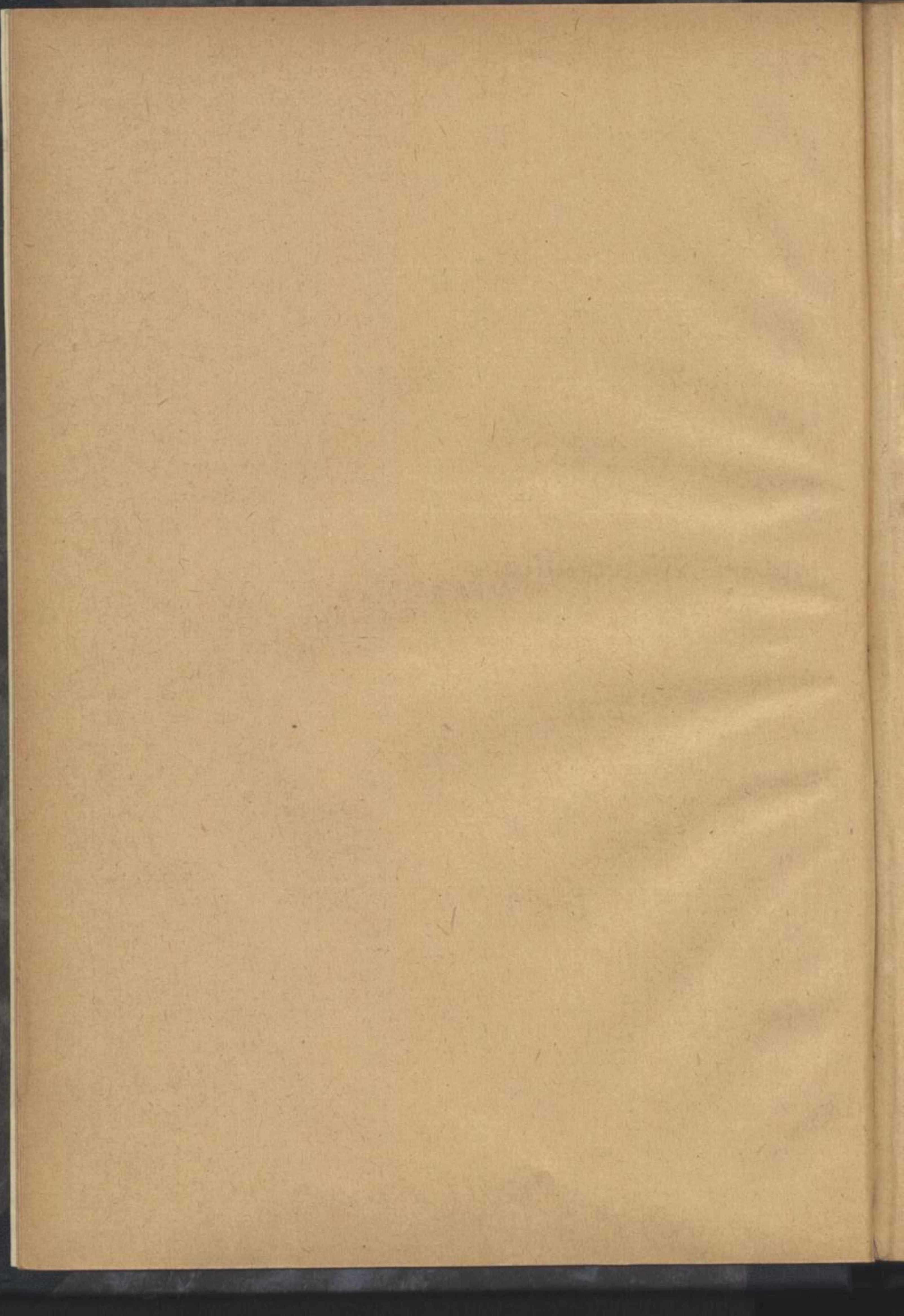

SLUB gefördert von der
Deutschen Forschungsgemeinschaft 


\section{Vorwort.}

Die vorliegende Schrift ist eine Ausführung der kurzen Mitteilung: „Die Palimpsestphotographie“, Sitzungsbericht XXXVII, 1914, der Königl. Preußischen Akademie der Wissenschaften, philosophisch-historische Abteilung.

Zweck dieser Abhandlung ist, durch eine systematische Darstellung der Palimpsestphotographie in ihren theoretischen Grundzügen und praktischen Anwendungen die noch ungehobenen Schätze der Palimpseste den Geschichtsforschern, Philologen u. a. zugänglich zu machen.

Für den Techniker, der über die Palimpseste selbst gern Näheres erfahren möchte, sei hier über die Entstehung und Wert der Palimpseste einiges gesagt.

In vergangenen Zeiten wurde nicht selten die ursprüngliche Schrift der Pergamenthandschriften weggescheuert und durch eine sowohl der Form als dem Inhalt nach neue Schrift ersetzt. Das Wort Palimpsest kommt von dem Griechischen $\pi \alpha \lambda \iota \mu \varphi \sigma \varepsilon \epsilon \tau 0 \varsigma$ ( $\pi \alpha \lambda \iota \mu \varphi \sigma \alpha \omega)$, und heißt wieder abgewaschen, abgescheuert. Die lateinische Bezeichnung „codex rescriptus" für Palimpsest hebt hervor, daß ein ursprüngliches Werk wieder überschrieben worden ist. Mitunter, wurde es aber zweimal überschrieben, so daß drei Schriften 'sich überlagern ${ }^{1}$ ).

Der Wert der eigentlichen, alten Palimpseste geht aus der Tatsache hervor, daß mehrere klassische Werke oder größere Teile derselben uns nur durch Palimpseste erhalten sind. Unika und berühmt sind, um nur wenige zu nennen: Gajus in Verona,

I) Ein Beispiel findet sich in der Abhandlung: Untersuchungsergebnisse einer doppelt reskribierten Wolfenbüttler Handschrift mittels der Fluoreszenzphotographie. Von P. A. Dold, O. S. B. Ztsch. f. Bibliothekswesen, S. 233 ff., 1917. 


\section{- VIII -}

Codex Ephremi in Paris, die gotische Bibelübersetzung von Ulfila in Wolfenbüttel, Plautus in Mailand, Cicero de republica in der Vatikana.

Die Entstehung der Palimpseste ist vor allem auf wirtschaftliche Gründe zurückzuführen. Das Pergament war einst ein teurer Schreibstoff. Sein Bedarf war groß, denn man benötigte es nicht nur für Bücher, sondern auch für den privaten brieflichen Verkehr, für wirtschaftliche und amtliche Mitteilungen, Bescheinigungen und Urkunden aller Art. Diese Zwecke forderten alljährlich große Mengen von Pergament, die besonders zu Kriegszeiten und Zeiten der Teuerung schwer zu beschaffen waren.

Wenn daher von irgendeinem Werk ein Duplikat vorhanden war, das wenig schön oder fehlerhaft geschrieben, so entfernte man die Schrift, um das Pergament zu einem neuen, den jeweiligen Bedürfnissen entsprechenden Werk zu gebrauchen. Gleiches geschah, wenn der Inhalt der Schrift einen unmittelbar praktischen Wert nicht mehr darstellte. Dies war z. B. der Fall, wenn das Recht mit dem Kommen und Gehen der Völker, mit dem Entstehen und Vergehen ihrer Staaten sich änderte, oder wenn Schriften, deren Wortlaut von Amts wegen der Willkür dès Einzelnen nicht überlassen werden konnte, eine Neufassung erhielt. Auf diese Weise verdrängte die Vulgata die Italia usw.

Die Zahl der vorhandenen und zum guten Teil noch ungelesenen Palimpseste ist eine ziemlich große, größer als allgemein angenommen wird. Der Verfasser hat ein Verzeichnis derseiben für seinen Gebrauch hergestellt. Wenngleich dieses Verzeichnis noch unvollständig ist, so weist es doch bereits mehr als 300 Codices, zum Teil sehr umfangreiche Palimpseste in lateinischer, griechischer und verschiedenen orientalischen Sprachen auf. Es darf mit Bestimmtheit gesagt werden, daß neben "Ausgrabungen" die Palimpsestphotographie die ergiebigsten wissenschaftlichen Quellen der klassisehen und kirchlichen Frühzeit und des hohen Mittelalters zu erschließen geeignet ist. 


\section{$-\mathrm{IX}-$}

Überaus groß ist die Zahl der Kleinpalimpseste. In vielen Pergamenthandschriften, von denen die größeren Bibliotheken hunderte, ja tausende besitzen, finden sich zahlreiche, mitunter durch ganze Werke systematisch durchgeführte Einzelradierungen.

Es sind beispielsweise vermeintliche grammatikalische Verbesserungen. Durch sie wurde nicht selten der Wert, die Verwandtschaft oder die Abhängigkeit des ersten Textes von anderen verdeckt.

In anderen Fällen wurden vermeintliche sachliche Verbesserungen vorgenommen, und zwar an Hand anderer Handschriften, denen man größere Glaubwürdigkeit zumaß. Auch hier wurde nicht selten das Gegenteil von dem geschaffen, was beabsichtigt war. Der Text, der der Originallesung nahekam oder sich mit ihr deckte, wurde durch irreführende Varianten ersetzt.

Mitunter ist eine Rasur allein in einem ganzen Werk für seine Beurteilung von ausschlaggebender Bedeutung. Dies gilt hauptsächlich von dem Namen des Verfassers oder des einstigen Besitzers. Seine Wiedergewinnung löst nicht selten textgeschichtliche oder textkritische Fragen, die sonst kaum oder gar nicht entschieden werden können. Es finden sich aber auch absichtliche oder erzwungene Fälschungen vor. Vor ein paar Jahrzehnten wurde selbst von namhaften Forschern solchen einzelnen formellen und sachlichen Veränderungen nicht die gebührende Aufmerksamkeit gewidmet.

Heute wendet man ihnen ein besonderes Augenmerk zu. Der Gelehrte, der sich der Erforschung der historischen Quellen widmet, wird daher jedes technische Hilfsmittel, das ihm den ursprünglichen Text soweit als möglich wiederzugeben vermag, willkommen heißen. Ja, er wird das dritte Auge des Unsichtbaren nicht mehr entbehren können, wenn die Ergebnisse seiner Forschung nicht ebensoviel Fragezeichen aufweisen sollen als das Original Rasuren.

In der vorliegenden Schrift soll dann insbesondere die Anwendung der Palimpsestphotographie zur Untersuchung 
moderner, forensischer Schriftstücke gezeigt werden, wobei der unsichtbaren Anfärbung nur kurz gedacht wird. Gerade auf forensischem Gebiet werden die neuen photographischen Verfahren eine breitere Anwendung finden und auch dann dauernden Wert behalten, wenn der letzte Buchstabe der alten Palimpseste schon erschlossen ist.

Ein Teil dieser Schrift ist auch den älteren Verfahren gewidmet, nicht nur, weil sie in vereinzelten Fällen heute noch Anwendung finden, sondern weil der fundamentale Unterschied des Fluoreszenzverfahrens, der eigentlichen Palimpsestphotographie, von dem Verfahren der älteren Technik durch eine inhaltlich-genetische Gesamtdarstellung am deutlichsten zum Ausdruck gebracht wird.

Die vorliegende Abhandlung muB und darf die Kenntnis der gewöhnlichen Photographie und eine gewisse Vertrautheit mit den Grundzügen der Physik und Chemie voraussetzen. Zur Einführung in die besondere Photographie der Handschriften seien empfohlen:

Die Photographie im Dienste der Geisteswissenschaften. Von Prof. Dr. A. Krumbacher, Leipzig 1906; Die Photographie historischer Dokumente. Beiheft zum Zentralblatt für Bibliothekswesen. Von P. R. Kögel, O. S. B., Leipzig 1915. - Weitere Literaturangaben bis zum Jahre 1902 finden sich im Handbuch der Bibliothekslehre. Von Dr. A. Grassel, S. 43う̄, Leipzig 1902.

Zur Einführung in die Photographie forensischer Handschriften dient:

Lehrbuch der gerichtlichen Photographie, Bd. II. Nachweis von Schriftfälschungen usw. unter besonderer Berücksichtigung der Photographie. Von Dr. G. Baumert, M. Dennstedt und F. Voigtländer, Braunschweig 1906.

Beuron, Hohenzollern, 1920.

Der Verfasser. 


\section{Inhaltsverzeichnis.}

Vorwort

I. Die geschichtliche Entwicklung der Palimpsestphotographie .

2. Die Palimpsestphotographie mit reflektierten, sichtbaren Strahlen 2

Die Beleuchtung . . . . . . . . . . . . . 3

Die Lichtfilter . . . . . . . . . . . . . . . 5

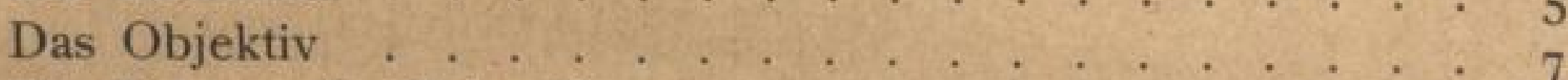

Die lichtempfindliche Platte . . . . . . . . . . . . . 7

3. Die Palimpsestphotographie mit reflektierten, urisichtbaren Strahlen

Die Aufnahme mit Strahlen von $366 \mu$ Wellenlänge

Die Aufnahme mit Strahlen von $3^{1} 3 \mu \mu$ Wellenlänge

4. Die Wiedergabe des Palimpsestes durch Fluoreszenz (nicht reflektierte Strahlen) bei ultravioletter Beleuchtung . . . I7

Die Erzeugung der Fluoreszenz durch spektrales Ultraviolett im allgemeinen

Die Lichtquelle und Einzelnbestandtele der ultravioletten Projektionseinrichtung . . . . . . . . . . . . Ig

Der Phosphoreszenz- und Fluoreszenzschirm ..... . . 27

Das Spektrum . . . . . . . . . . . . . . . . . 28

Die Erzeugung der Fluoreszenz mit spektralem Ultraviolett von $3^{1} 3 \mu \mu$ und mit Silberfilter

Die Erzeugung der Fluoreszenz mit spektralem Ultraviolett von $366 \mu \mu$ und dem Blauuviolfilter . . . . . . . . $3^{2}$

Das Objektiv . . . . . . . . . . . . . . . 35

Die lichtempfindliche Platte. . . . . . . . . . . . $3^{6}$

5. Das Ultraviolett-Absorptionsfilter . . . . . . . . . . . 37

6. Zusammenfassender Vergleich der Ausführungsmittel des Ultraviolett-Fluoreszenzverfahrens . . . . . . . . 43

Die Schriftausbeute . . . . . . . . . . . . . . . . . 44

7. Versuche der Erzeugung der Fluoreszenz mit subultravioletten und korpuskularen Strahlen ........... . . 48

Versuche mit den (subultravioletten) Röntgenstrahlen . . . $4^{8}$ 
Versuche mit Teslastrahlen . . . . . . . . . . . 49

Versuche mit Kathodenstrahlen . . . . . . . . . . 50

Versuche mit Anoden- bzw. Kanalstrahlen . . . . . . $5^{I}$

Versuche mit Radium . . . . . . . . . . . . . . 52

8. Die Verfahren zum Abdecken der Sekundärschrift der Codices rescripti . . . . . . . . . . . . . . . . . . 53

9. Die unsichtbare Anfärbung . . . . . . . . . . . . 54

10. Erläuterungen der Beispiele der Palimpsest- und forensischen Fluoreszenzphotographie .. . . . . . . . . . $5^{6}$ 


\section{Die geschichtliche Entwicklung der Palimpsestphotographie.}

Die Palimpsestphotographie erscheint zuerst als eine dem besonderen Gegenstand angepaßte Anwendung der allgemeinen Grundsätze der Photographie.

Grundsatz des Verfahrens ist die photographische Aufnahme der Handschrift bei einer intensiven Beleuchtung mit sichtbaren Strahlen, die vom Pergament reflektiert, von der Schrift aber absorbiert werden.

Die Anwendung dieses Verfahrens findet heute noch in vereinzelten Fällen statt, so z. B. zur Wiedergabe von Schriften, die in den Farbflecken chemischer Reagenzien liegen u. dgl.

Das Verfahren der Wiedergabe durch reflektierte Strahlen erhielt eine Erweiterung durch die Beleuchtung der Vorlage mittels ultravioletter Strahlen ${ }^{1}$ ), unter Beibehaltung der Abbildung des Objektes durch die vom Planum zurückgeworfenen Strahlen.

Die eigentliche Palimpsestphotographie entstand aber erst durch Einführung einer neuen Grundlage, der Lumineszenz. Das Objekt bildet sich nicht mehr durch reflektiertes Licht, sondern durch Selbstleuchten infolge Erregung durch fremdes auffallendes Licht ab. Dadurch wurde die Palimpsestphotographie zu einer eigenen Technik, deren besonderer Gegenstand die photographische Sichtbarmachung unsichtbarer (aber vorhandener) alter und neuer Schriften ist ${ }^{2}$ ).

. I) Die Photographie historischer Dokumente, 1. c., S. 77. Ztsch. f. Reproduktionstechnik 1913, S. 42 u. 58 . Umschau 18 , S. 648, 1914.

2) D. R.P. $27403^{\circ}$ vom 30. Okt. 1913 und D. R. P. 288327 vom II. Okt. I9I4 (Inhaber: P. R. Kögel). Es ist selbstverständlich, daß die Verfahren immer längere Zeit vor der Patentanmeldung technisch ausgearbeitet waren.

Kög e 1, Die Palimpsestphotographie. 
Die Erzeugung der Lumineszenz, in Form von Fluoreszenz, findet mit ultravioletten Strahlen statt $^{1}$ ).

Die Umgestaltung der späteren Neuschrift der Codices rescripti aus ihrem Schwarzwert in einem dem Planum nahekommenden Helligkeitswert zur besonderen Hervorbebung der Erstschrift ist Gegenstand eines eigenen Nebenverfahrens. Dieses steht mit der Wiedergabe der Erstschrift aber in keinem notwendigen, sondern nur äußeren Zusammenhang.

Das hohe Verdienst, ein solches Verfahren zuerst geschaffen zu haben, gebührt O. Gradenwitz und E. Pringsheim ${ }^{2}$ ). Dieses Verfahren wurde später von 0 . Mente und A. Warschauer, dann von R. Kögel weiter ausgebaut ${ }^{3}$ ).

Im Laufe der Zeit wurde selbstverständlich auch von anderer Seite versucht, das Problem der Palimpsestphotographie zu lösen. Diese Versuche waren indes erfolglos oder sind heute überholt. Mitunter fehlen den Veröffentlichungen solcher Versuche auch die erforderlichen technischen Angaben $\left.{ }^{4}\right)$. Einige solcher Versuche werden in Kapitel 7 näher besprochen.

\section{Die Palimpsestphotographie mit reflektierten, sichtbaren Strahlen.}

Dieses Verfahren, das das älteste ist, findet heute noch, wie früher erwähnt, Anwendung zur Wiedergabe von Palimpsesten mit sichtbar erhaltener Erstschrift und von solchen Schriften, die in den sichtbaren oder unsichtbaren Flecken chemischer Reagenzien liegen.

I) Vgl. die vorläufige Mitteilung: Sitzungsbericht der Kgl. preuß. Akademie, XXXVII; 19I4, 29. Okt. Philosoph.-histor. Klasse. Von P. R. Kögel, O. S. B.

2) Verhandlungen der Physikal. Gesellschaft zu Berlin, Bd. 3 , I894, S. $5^{8}$.

3) Siehe Kapitel 8.

4) Rivista delle biblioteche e degli archivi. I-4, I9I5. 


\section{Die Beleuchtung.}

Bekanntlich erscheint eine helle Fläche, wie die eines Pergamentes, dadurch weiß, daß sie auffallendes, "weißes" Licht zurückwirft. Ein ,dunkler" Körper, wie die Schrift auf dem Pergament oder Papier, erscheint schwarz, weil er das sichtbare, auffallende Licht in dem Maße absorbiert, daß die Menge der von ihm zurückgeworfenen Strahlen im menschlichen Auge keine merkbare Lichtempfindung auszulösen vermag. Das Reflexionsvermögen des weißen Papieres beträgt etwa $90 \%$, dasjenige der Druckerschwärze etwa $10 \%$. Die Schrift stellt also ein teilweises, optisches Vakuum dar.

Der photographisch wirksame Gegensatz zwischen Untergrund und Schrift kann durch eine intensive Beleuchtung mit solchen Strahlen gesteigert werden, die vom Untergrund reflektiert, von der Schrift dagegen absorbiert werden, wobei die besondere Farbenempfindlichkeit der Platte mit den reflektierten Strahlen in Übereinstimmung sich befinden muß.

Die Beleuchtung muß kräftig sein, weil nicht der bloße Farbenunterschied zwischen Schrift und Pergament, sondern die Größe dieses Unterschiedes die höchsten Gegensätze im Negativ bewirken kann.

Starke Lichtquellen, die eine solche Beleuchtung ermöglichen, sind elektrisches Bogenlicht, Quecksilberdampflicht und das hochkerziger Metalldrahtlampen. Das Nernstlicht gehört auch zu den starken Lichtquellen. Durch Bruch der Leuchtstifte versagt es aber leicht.

Zur Beleuchtung der Vorlage mit Bogenlicht werden die Lampen ${ }^{\text {i) }} \mathrm{zu}$. beiden Seiten des Originals aufgestellt. Wenn

I) Nähere Beschreibungen und Abbildungen der sogenannten Reproduktionslampen können hier unterbleiben, da sie sich nur der Form nach von den bekannten gewöhnlichen Bogenlampen unterscheiden. Eingehendes kann aus dem Lexikon f. Photographie u. Reproduktionstechnik von Professor G. H. Emmerich, S. I42 ff., I9I0, entnommen werden, sowie aus den leicht erhältlichen Preislisten der Fabriken. 
bei sehr sparsamem Stromverbrauch die für den vorliegenden Zweck erforderliche Lichtfülle erzielt werden soll, kann das Quecksilberdampflicht empiohlen werden.

Die Quecksilberdampflampe ${ }^{1}$ ) (Abb. 1, $g g^{\prime}$ ) besteht aus einem Vakuumrohr, dessen Länge $40-125 \mathrm{~cm}$ beträgt. Am oberen Ende des Leuchtrohres befindet sich die positive Eisenelektrode, am unteren in einer Kühlkammer Quecksilber, das. die negative Elektrode ist. In den Stromkreis wird ein Widerstand und eine Selbstinduktionsspule geschaltet. Die Zündung erfolgt handtätig oder automatisch durch Kippung. Die Lampen brennen (obne Transformator) nur mit Gleichstrom. Die Lichtstärke beträgt bei einer Länge des Leuchtrohres von $11 \overline{\mathrm{cm}}$ 1000 Kerzen, bei einer von $55 \mathrm{~cm}$ Länge 500 Kerzen. Nur die größere Lampe ist zu einer gleichmäßig starken Beleuchtung von Handschriften zu gebrauchen, da nur die obere Hälfte des Rohres gleichmäßiges Licht ausstrahlt. Von der Mitte bis zur Kathode tritt ein (für das-Auge nicht erkennbarer) Lichtabfall ein.

Die Quecksilberdampflampen verlangen keine Erneuerung des Elektrodenmaterials, hedürfen keiner Wartung wäbrend des Betriebs. Ihre Brenndauer übersteigt meist 1000 Stunden.

Weniger sparsam im Stromverbrauch als die Quecksilberdampflampen sind die hochkerzigen Metalldrahtlampen ${ }^{2}$ ). Ihr Licht ist aber viel reicher an gelben und roten Strahlen als das der Quecksilberlampen. Sie besitzen die Form gewöhnlich elektrischer Glaslampen, sind aber größer (Abb. 1d). Ihre größte Brenndauer ist die von etwa 800 Stunden. Der Anschaffungspreis ist um ein Mehrfaches geringer als der der Quecksilberdampflampen.

Zur Beleuchtung kleiner Schriftstücke, wie die forensischer Natur, eignen sich besonders gut die sogenannten Mikroskopier-

I) Westinghouse Cooper-Hewitt Cie., Berlin SW 48 .

a) Azolampe der Auer-A.-G.; Nitralampe der A.E. G.; WotanHalbwattlampe von Siemens-Schuckert. 
bogenlampen $\left.{ }^{1}\right)(A b b .2)$. Es wäre nur wünschenswert, daß an ein und derselben Lampe das Auswechseln des Kollektors aus gewöhnlichem Glas gegen einen aus weißem Uviolglas oder aus Quarz und das Anbringen der Blauuviolglasküvette möglich wäre ${ }^{2}$ ). Dies könnte mit geringen Aenderungen, die keine konstruktive Schwierigkeiten begegnen können, erreicht werden.

Das in Abb. 3 dargestellte Instrumentarium ist mit einer Quarzlampe ausgestattet, die einzig allein alle ultravioletten Strahlen liefert, die zur Ultraviolett- und zur Fluoreszenzphotographie in allen ihren Ausführungsformen erforderlich sind. Sie ist neben den anderen starken Lichtquellen immer notwendig.

\section{Die Lichtfilter.}

Der im Objekt durch diese zweckmäßige Beleuchtung geschaffene Farbenunterschied kann durch Filter ${ }^{3}$ ), die in den Strahlengang zwischen Objekt und Platte (am besten unmittelbar vor dem Objektiv) zu bringen sind, erhöht werden. Die Farbe des Lichtfilters soll annähernd komplementär zur Erstschrift sein, und zwar nach der subtraktiven Farbmischung.

Da die Erstschrift, soweit sie sichtbar ist, meist gelbrötlich ist, so wäre ein blaues Lichtfilter zu wählen. Ist das Pergament weiß und liegt die Schrift in keinem Farbflecken chemischer Reagenzien, so kann in diesem Falle aber ein Lichtfilter entbehrt werden, da die photomechanische oder die Bromsilberplatte durch ihre Unempfindlichkeit für Rot und besondere Empfind-

I) Zeif-Jena, Leitz-Wetzlar, Reichert-Wien u. a.

2) Siehe Näheres in Kapitel 4 .

3) Die photographischen Lichtfilter von A. v. Häbl, Verlag von Wilhelm Knapp in Halle (Saale), rgro. - Über die Verwendung des Quecksilberlichtes für mikroskopische Arbeiten (Herstellung monochromatischer Lichtfilter). Von A. Köhler; Ztsch. f. wissenschaftl. Mikroskopie, XXVII, I9ı, S. 329. - Über Farbgläser für wissenschaftliche und technische Zwecke. Von Dr. R. Zsigmondy. Über Jenenser Lichtfilter. Von Dr. R. Grebe. Ztsch. f. Instrumentenkunde. Hełt 4 , rgor, S. ror. 
lichkeit für Blau die Aufgabe des Lichtfilters, die Auswahl eines Teilfarbenbildes, selbst übernimmt.

Unumgänglich notwendig ist dagegen ein Lichtfilter zur Wiedergabe von Schriften, die in einem für die stets vorwiegend blauempfindliche Platte ${ }^{1}$ ) unaktinischen Planum, z. B. in den Flecken des "Gallustinktur"-Reagens liegen. Die braune Farbe eines solchen Fleckens reflektiert nur wenig Blau. Zur Erreichung der notwendigen Deckung im Planum des Negativs müßte deshalb die Belichtung auf einer gewöhnlichen Platte um ein Mehrfaches verlängert werden. Dadurch würde aber auch das von der Schrift zurückgestrahlte Licht auf der Platte wirksam werden und eine Teilschwärzung in der darauffolgenden Entwicklung zeitigen. Auf der Platte würde also der erforderliche Gegensatz zwischen Schrift und Untergrund nicht erscheinen. Daher muß das von der Schrift reflektierte blauviolette Licht durch ein gelbes Filter ${ }^{2}$ ) absorbiert, d. h. auf ein unwirksames Minimum herabgedrückt werden. Dadurch wirkt auch das vom Untergrund zurückgeworfene Licht auf die Platte, die zweckentsprechend gelbempfindlich sein muß (orthochromatis(che Zeitplatte $\left.{ }^{3}\right)$ ).

Das Lichtfilter ist entweder, wie die bekannten Gelbscheiben, fest, oder flüssig. Der Farbstoff wird im letzteren Falle mit Wasser oder Alkohol gelöst ${ }^{4}$ ), in eine Glasküvette gegeben und diese unmittelbar vor das Objektiv gebracht. Wenn an die Bildschärfe der Aufnahme hohe Ansprüche gestellt werden, müssen die Küvetten aus „optischem" Glas be-

1) Man verwendet daher am besten die sogenannten filterlosen, farbenempfindlichen nassen Emulsionen.

2) Ein Gelbfilter, das fast in allen solchen Fällen ausreicht, ist das Trockenfilter "mit sechsmal verlängerter Exposition" von Hoh \& Hahne, Leipzig.

3) Silber-Eosinplatte von O. Perutz, München u. a. oder die nassen Platten.

4) Rezepte und Tabellen von Dr. J. M. Eder, 8. Aufl., Verlag von Wilhelm Knapp in Halle (Saale), S. $5^{8}$. 
stehen. Für den Privatgebrauch genügen meist solche aus Spiegelglas, wenn der innere Abstand der parallelen Wände nicht mehr als $5 \mathrm{~mm}$ beträgt (Abb. 4).

\section{Das Objektiv.}

Von einem guten Reproduktionsobjektiv wird verlangt, daß es sphärisch, anastigmatisch und ehromatisch korrigiert sei. Es muß bei mittlerer Blende das aufzunehmende Format in Naturgröße scharf bis an den Rand auszeichnen. Das Öffnungsverhältnis zur Brennweite beträgt bei solchen Objektiven gewöhnlich F:9-12. Die Brennweite des Objektivs soll die Diagonale des Formates ausmessen. Solche Objektive werden Apochromate genannt. Meist würde aber ein (viel billigerer) Aplanat genügen, der nur für die blanen und gelben Strahlen korrigiert ist, wodurch die Brennpunkte dieser Strahlen in eine die Bildschärfe wenig beeinträchtigende Aberrationszone fallen.

Diese Objektive sind jedoch lichtschwach und eignen sich für die Palimpsestfluoreszenzphotographie, die sehr lichtstarke Objektive verlangt, nicht. Man wird daher - außer, es seien reiche Mittel vorhanden - keines der obengenannten, im übrigen vortrefflichen Reproduktionsobjektive erwerben, sondern eines der im Kapitel 4 bezeichneten Objektive.

In der forensischen Praxis wird das Objekt häufig vergrößert. Die Aufnahme erfolgt mit einem sogenannten Mikroobjektiv ${ }^{1}$ ). Es findet auch in der Fluoreszenzphotographie Vérwendung. Eine $20-30$ fache Vergrößerung (bei einer Brennweite von etwa $50 \mathrm{~mm}$ des Objektirs) wird fast immer zur Klarlegung des Untersuchungsgegenstandes genügen.

\section{Die lichtempfindliche Platte.}

Ein von der Vorlage zurückgeworfener Strahl, der durch das Objektiv auf die in der Kamera befindliche lichtempfindliche Platte gelangt, erzeugt in einer bestimmten Zeit eine

I) Planare (Z eiß-Jena) u. dgl. 
chemische Veränderung, die zunächst unsicbtbar ist, durch die Entwicklung, d. h. durch die Reduktion des Halloidsilbers zu metallischem Silber, aber ein sichtbares Bild liefert. Das nicht reduzierte Halloidsilber wird durch das sogenannte Fixieren mit unterschwefligsaurem Natron aufgelöst und geht ins Lösemittel über. Dieses muß aus der Platte ausgewaschen werden. In dem fertigen Negativ entspricht der hellen Fläche des Originals eine dunkle Fläche und der Schrift eine mehr oder weniger helle Fläche.

Die Abbildung der Teillichter wird im wesentlichen durch die Gradation der lichtempfindlichen Substanz bestimmt. Bei langer Gradation weist der Übergang von Hell zu. Dunkel eine Anzahl Zwischenstufen auf, während bei kurzer Gradation der Übergang von Hell zu Dunkel ein schroffer ist.

Sehr geringe Helligkeitsunterschiede, wie schwache Schriftspuren auf einem gelblichen Pergament es sind, werden nicht selten durch eine Platte langer Gradation deutlicher wiedergegeben als durch eine Platte geringer Abstufung.

Wie bereits erwähnt, muß die besondere Farbenempfindlichkeit der Platte mit der Farbe der Strahlen übereinstimmen, die vom Planum am meisten, von der Schrift am wenigsten reflektiert werden.

Daher wird eine orthochromatische Zeitplatte zur Aufnahme von Schriften in einem gelbbraunen Planum benutzt, so z. B. mit einem starken Gelbfilter zur Wiedergabe der Schrift in den Flecken der "Gallustinktur". Die teilweise sichtbaren Schriften in unsichtbaren Flecken des Schwefelammoniums und anderer Schwefelsalze, die mit Hilfe der Fluoreszenz sofort zu erkennen sind ${ }^{1}$ ), werden ohne Lichtfilter mit einer photomechanischen oder mit einer gewöhnlichen Bromsilberplatte aufgenommen.

I) Die stets schädliche Anwendung solcher Reagentien kann auf diese Weise sehr leicht festgestellt werden. 


\section{Die Palimpsestphotographie mit reflektierten, unsichtbaren Strahlen.}

In dem vorher beschriebenen Verfahren erhielt das Negativ seine Zeichnung durch die vom Planum reflektierten sichtbaren Strahlen. Sehr schwache Schriftspuren werfen aber häufig gleiche Mengen sichtbarer Strahlen zurück wie das Planum, so daß eine Differenzierung beider auf diesem Wege unmöglich ist.

Es ersteht sich daher die Frage, ob zwei Objekte, die gleiche Mengen sichtbarer Strahlen reflektieren, dies auch für unsichtbare Strahlen vermögen.

Unsichtbare Strahlen, die sich unmittelbar an das sichtbare Spektrum anreihen, sind Infrarot ${ }^{1}$ ) und Oltraviolett.

Palimpsestaufnahmen mit infraroten Strahlen hat der Verfasser mit Hilfe des Woodschen Infrarotfilters und einer Pinacyanolplatte ${ }^{2}$ ) ausgeführt. Die Versuche zeigten, daß die Erstschrift nur etwas weniger Infrarot reflektiert als das Pergament. Dies entsprach auch den Erwartungen, die durch theoretische Gründe gegeben waren.

Die Absorptionsfähigkeit der Erstschrift nimmt nämlich bereits im sichtbaren Spektrum mit abnehmender Wellenlänge zu. Daraus war zu schließen, daß die Reflexionsfähigkeit der Schrift mit der gröBeren Wellenlänge der Beleuchtungsstrahlen zunehmen würde, was durch Versuche bis zu einem gewissen Grade bestätigt wurde.

Die gleichen Gründe, die die Infrarotreflexion der Schrift erwarten ließen, drängten zur Annahme, daß die ultravioletten Strahlen absorbiert würden. Dies trifft auch zu. Aber das

I) Über die bisherigen Beobachtungen im ultraroten Spektrum. Von Dr. W. Beetz, 190\%. - Arch.Sc. phys. et nat. Genève, T. XXXIX, Bd. 3, S. 270; 1915. - Ber. d. Akad. Wien. II. Bd. I24, S. 23I-240, I915.

2) Von der Trockenplattenfabrik Westendorp \& Wehner, Köln a. Rh. 
Pergament absorbiert ebenfalls kräftig Ultraviolett, wenn auch weniger als die Erstschrift.

Wenn das Pergament gut weiß ist, wird mit der Ultraviolettphotographie öfters mehr Text zutage gefördert als mit sichtbaren Strahlen. Der Gewinn ist jedoch meist gering und erreicht nicht annähernd den des später eingehend beschriebenen Fluoreszenzverfahrens.

Für die Untersuchung moderner, forensischer Schriftstücke oder auch von älteren Objekten, wie Miniaturen u. a., wird die Ultraviolettphotographie in einzelnen Fällen dauernden Wert aufweisen. Bemerkenswerte Versuche sind Professor O. Mente und Dr. J. Richter zu verdanken ${ }^{1}$ ).

In diesem wie in folgenden Kapitel, das dem Fluoreszenzverfahren gewidmet ist, wird die Aufnahmetechnik in zwei Abschnitten besprochen.

Für diese Zweiteilung waren einerseits die Hauptgruppen der ultravioletten Strahlen der Quecksilberquarzlampe als der wichtigsten Beleuchtungsquelle und andererseits die Ultraviolettfilter maßgebend.

Die erste Hauptgruppe des Quecksilberquarzlichtes umfaßt die Strahlen von $366 \mu \mu$ und des angrenzenden Spektrums. Ein diese Strahlen durchlassendes Filter ist die später näher beschriebene Blauuviolglasküvette. Dieses Filter mit Füllung läßt im allgemeinen mehr oder weniger die Strahlen von $400-300 \mu \mu$ durch, absorbiert aber die sichtbaren Strahlen. Sein Durchlässigkeitsmaximum befindet sich bei $350 \mu \mu^{2}$ ).

I) Die Photographie historischer Dokumente, 1. c., S. $76 .-$ D. R. P. 274 O30. - Ztsch. f. Reproduktionstechnik, S. 42 u. 58, I9I3. Umschau, Bd. 18 , S. 6,8 , I9I4.

2) Über andere Ultraviolettfilterfarbstoffe siehe: Absorption und Sensibilisierungsvermögen einiger gelber Farbstoffe. III. Teil in Beiträge zur Photochemie und Spektralanalyse. Von J. M. Eder und E. Valenta. I904. Verlag von Wilhelm Knapp, Halle (Saale), und E. Lechner, Wien, - Quantitative Lightfilter for the Ultra-violet Part of Spectrum. Journ. Phys. Chem., Bd. 2I, S. $3^{82-401, ~ 1917 . ~}$ Journ. Chem. Soc., Bd. II2 [2], S. 349, I9I7. Die letztgenannten Ab- 
Die obere Grenze der ultravioletten Strahlen, die von dem genannten Filter durchgelassen werden, liegt bei $400 \mu \mu$. Strahlen größerer Wellenlänge werden bereits zum sichtbaren Spektrum gezählt ${ }^{1}$ ). Die untere Grenze kann man auf etwa $335 \mu \mu$ legen, da für Strahlen kürzerer Wellenlänge die Durchlässigkeit der Blauuviolglasküvette stark abnimmt. Sie wird dann durch ein anderes Filter ersetzt, das mit der zweiten Hauptgruppe des Quecksilberdampflichtes in Verwendung kommt.

Die zweite Hauptgruppe des ultravioletten Spektrums des Quecksilbers umfaßt die Strahlen von $313 \mu \mu$ Wellenlänge und nächst kürzere. Die Strahlen werden bei Absorption des sichtbaren Lichtes durch ein Silberfilter durchgelassen.

In diesem Abschnitt werden dann auch die anderen Lichtquellen besprochen, deren ultraviolette Strahlen von dem genannten Filter in größerer Menge freigegeben werden. Der Einfachheit wegen werden die beiden Teilabhandlungen nach den zwei Hauptgruppen der Strahlen von $\lambda=366 \mu \mu$ und $313 \mu \mu$ benannt.

\section{Die Aufnahme mit Strahlen von $366 \mu \mu$ Wellenlänge.}

Große Mengen von ultravioletten Strahlen liefert Nickelbogenlicht. Die wichtigsten Linien des ultravioletten Emissionsspektrums von Nickel sind die von $386,383,380,356,352$, 349, $341 \mu \mu$. Die Anordnung der Elektroden ist folgende: Die Kohle ${ }^{2}$ ) der oberen, positiven Elektrode enthält die Nickelsalze. Die untere, negative Elektrode besteht bei Gleichstrom aus einer gewöhnlichen Kohle, bei Wechselstrom aber auch aus einer Nickeldochtkohle.

handlungen konnte ich noch nicht einsehen. Studies of the ultraviolet Transparency of certain coloured Media. Phil. Mag. (6), Bd. 33, S. $450-456$, I9I7. Neueste Veröffentlichungen: Ztsch. f. wissenschaftl. Photographie Bd. 18, S. 235 ; Bd. 19, S. 57.

I) J. Plotnikow, Photochem. Versuchstechnik, S. 274.

2) Siemens-Schuckert. 
Eine andere sehr brauchbare Lichtquelle ist die Uviollampe von Schott u. Gen., Jena. Sie weist die Form der früher beschriebenen W.-C.-H.-Lampe auf. Das Leuchtrohr besteht aus dem für Ultraviolett von größerer Wellenlänge durchlässigen Bariumphosphatkronglas und gibt reichlich Strahlen von $366 \mu \mu$, was bei der W.-C.-H.-Lampe nicht der Fall ist, da ihr Leuchtrohr aus dem Ultraviolett stark absorbierenden Bleiglas besteht.

Zur Beleuchtung der Vorlage mit vertikaler Aufstellung der Lampen muß auch hier der obere Teil der Röhren benutzt werden, da von der Mitte $a b$ ein Lichtabfall stattfindet ${ }^{1}$ ).

Als die beste Lichtquelle muß die Quarzlampe bezeichnet werden, nicht wegen einer gleichmäßigen Lichtverteilung, die zu wünschen übrigläßt und die nur durch Anwendung mehrerer Lampen ausgeglichen werden kann, sondern wegen des gleichzeitigen hohen Gehaltes des Lichtes an Strahlen von 366 und von $313 \mu \mu$.

Bei der Aufnahme des Originals mit den Strahlen von $366 \mu \mu$ kann dasselbe mit einer dünnen Platte aus gewöhnlichem Glas glattgehalten werden, da die Strahlen von $\lambda=$ $366 \mu \mu$ durch Glas von etwa Millimeterdicke nur wenig absorbiert werden.

Vor oder unmittelbar hinter dem Objektiv wird das Lichtfilter (Abb. 5) gebracht. Die Blauuviolglasküvette hat hier auch die Aufgabe, etwa vorhandene Fluoreszenz ${ }^{2}$ ) des Originals unwirksam zu machen.

Die scharfe Einstellung des Bildes auf der Mattscheibe bzw. Platte erfolgt experimentell, wenngleich sie durch rechnerische Konstanten ermittelt werden könnte. Dureh das

I) Vgl. auch Photochem. Versuchstechnik. Von Joh. Plotnikow, Leipzig I9I2, S. 6.

2) Das Lichtfilter muß in diesem Falle stets unmittelbar sich am Objektiv befinden, welche auch die Art der Beleuchtung sei, mit freiem Licht, durch Filter oder mit spektralem Ultraviolett. 
Glas der K̈̈vette und die darin enthaltenen Farbstoffe wird die Brennweite im Bilde etwas verlängert, durch die Brechbarkeit der Strahlen etwas verkürzt.

Als Objektiv kann meist ein guter Anastigmat aus gebräuchlichem optischen Glas gebraucht werden. Die Ultraviolettdurchlässigkeit des' optischen Glases ist aber etwas verschieden. Flintglas absorbiert im allgemeinen das Ultraviolett stärker als Kronglas. Die Absorption des schweren Baryt-Kron ist jedoch erheblich größer als die des Baryt-Lichtflint. Das schwerste Silikat-Flint zeigt dagegen einen auffallend hohen Absorptionsfaktor ${ }^{1}$ ). Entsprechende Angaben in den Preislisten der Industrie wären erwünscht.

Die Aufnahme selbst erfolgt auf einer (photomechanischen) Chlorsilberplatte. Sie besitzt eine mittlere Empfindlichkeit ${ }^{2}$ ) für Strahlen von $355 \mu \mu$ mit einem Maximum bei etwa $380 \mu \mu$. Eine Bromsilberplatte ist nicht zu empfehlen, weil ihre Durchschnittsempfindlichkeit bei 430 und $480 \mu \mu$ liegt. Wegen der Ebene, die fast allen Ultraviolettbildern eigen ist, muß auch die kurzgradierte Chlorsilberplatte vorgezogen werden.

\section{Die Aufnahme mit Strahlen von $313 \mu \mu$ Wellenlänge.}

Die einzige Lichtquelle, die sehr kurzwellige Strahlen in großer Menge liefert, ist die Quarzlampe. An Stelle der Blauuviolglasküvette tritt ein Silberfilter. Es besteht aus einer dünnen Silberschicht, die chemisch auf eine Quarzplatte oder sehr dünne Weißuviolglasplatte niedergeschlagen wird. Am besten werden zwei solche Platten mit versilberten Innenflächen zu einem Filter $^{3}$ ) zusammengefügt.

I) Eder und Valenta, Denkschrift der k. Akademie, Wien 1894. Ztsch. f. Reproduktionstechnik, Heft 5, r9I7, S. 35.

2) Die Photographie bei künstlichem Licht usw. Von J. M. Eder, S. 29I, 19I2; Verlag von Wilhelm Knapp, Halle (Saale).

3) Bezugsquelle: C. Zeiß, Jena. 
Über die Ultraviolettdurchlässigkeit des Silbers sind in der Literatur ${ }^{1}$ ) sehr abweichende Angaben zu finden. Sie sind der Hauptsache nach auf die verschiedene Dichte der bei den Messungen angewandten Silberschichten, sowie auf die Verschiedenheit der angewandten Lichtquellen zurückzuführen. In allgemeinen erstreekt sich das Durchlässigkeitsmaximum des Silbers auf die Strahlen von $302-325 \mu \mu$ Wellenlänge. Bei Benutzung der Quarzlampe wird hauptsächlich die Linie von $313 \mu \mu$ durchgelassen. Dies kann mit der Zeißschen Ultraviolett-Projektionsvorrichtung leicht gezeigt werden. Wenn zwischen Kollektor und Spalt das Silberfilter gebracht wird, $\mathrm{so}^{\circ}$ ist auf dem Phosphoreszenzschirm nur mehr die Linie von $313 \mu \mu \mathrm{zu}$ beobachten, und zwar am gleichen Ort, an dem sie sich vor Zwischenschalten des Filters befand.

Die Strahlen von $313 \mu \mu$ werden durch gewöhnliches Glas in der Dicke von-wenigen Millimetern stark absorbiert. Daher kann das Original bei solchen Aufnahmen nicht mit einer gewöhnlichen Glasplatte glattgehalten werden. Auch sind die üblichen Objektive aus Glas zur Aufnahme nicht zu gebrauchen.

An Stelle des Glasobjektives tritt ein Objektiv aus Quarz ${ }^{2}$ ). Dieser ist für Strahlen von $313 \mu \mu$ und kürzere sehr durchlässig Das Quarzobjektiv hat die Eigenschaften eines Monochromaten, d. h. es hat für Strahlen verschiedener Wellenlänge einen verschiedenen Brennpunkt. Infolgedessen ist der optische Brennpunkt, der von dem menschlichen Auge hauptsächlich in den gelben Strahlen gewählt wird, nicht identisch mit dem photochemischen Brennpunkt der ultravioletten Strahlen. Dieser ist infolge der größeren Brechbarkeit der ultravioletten Strahlen

I) H. Ká y ser, Handbuch der Spektroskopie, Bd. 3, I905, S. $3^{\text {I9; }}$ J. M. Eder, Handbuch der Photographie, Bd, I, Teil 2, S. 197, und Teil 3, S. 339 .

2) Bezugsquelle: C. P. Goerz, Berlin-Friedenau. Vgl. dazu Eders Jahrbuch f. Photographie u. Reproduktionstechnik, I9I3, S. II2. - R. Neuha u B, Lehrbuch d. Mikrophotographie, Leipzig r9o7, S. r6r. 
gegenüber dem der sićhtbaren Strahlen verkürzt. Die Mattscheibe bzw. Platte ist daher dem Objektiv näherzubringen.

Man könnte auch die Vorderfläche des Objektivs versilbern, was für die Schärfe des Strahlenganges unter Umständen von Vorteil sein könnte. Da aber der Silberniederschlag, der sichtbares Licht nicht durchläßt, selbstverständlich auf dem Objektiv fest ist, so ist in diesem Falle eine visuelle Einstellung auf das gewünschte Format sehr erschwert. Diese müßte dann nach einigen festen, experimentellen Konstanten und rechnerischer Ermittlung erfolgen.

Die Aufnahme erfolgt auch hier mit einer photomechanischen Platte. Für Aufnahmen mit Strahlen unter $\lambda=220 \mu \mu$ hat Schuhmann ${ }^{1}$ ) bindemittelfreie Bromjodsilberplatten hergestellt. Miethe ${ }^{2}$ ) hat darauf aufmerksam gemacht, dafo wirklich bindemittelfreie Bromsilberschichten für Ultraviolett nahezu unempfindlich sind, und daß eine Unterlage eines organischen Kolloids die Empfindlichkeit bedeutend steigert.

Eder und Valenta ${ }^{3}$ ) haben optische Sensibilisatoren für Ultraviolett gefunden. So wirken z. B. Kanariengelb und Baumwollgelb im violetten und ultravioletten Teil sensibilisierend auf Trockenplatten. Bei Kanariengelb erhält man von dem Bande im grünen und blawen Teil, durch ein Minimum getrennt, ein breites Band bis $\lambda=c \cdot 300 \mu \mu$. Während Baumwollgelb das Minimum weiter gegen den weniger brechbaren Teil des Spektrums erkennen läßt, reicht die Sensibilisierung nur bis $\lambda=340 \mu \mu$, und verläuft das Band (wahrscheinlich infolge der Schirmwirkung) von der Fraunhoferschen Linie $K$ $(\lambda=393)$ bis $\lambda=430 \mu \mu$ ziemlich gleichmäßig.

I) Schuhmann, Sitzungsbericht der Kaiserl. Akademie der Wissenschaften, Wien I893, Bd. I02, II, a, S. 994. Eders Jahrbuch 1897 , S. 357 ; 1903 , S. 40 ; I909, S. $385 ; 1910$, S. 18.

2) Photogr. Korresp. 1909, S. 449 . Eders Jahrbuch I9I0, S. 486.

3) Photogr. Korresp. 1903 , S. 483 . Eder u. Valenta, Beiträge zur Photochemie u. Spektralanalyse, III. Teil, S. 164, 1904. Verlag von Wilhelm Knapp, Halle (Saale), und R. Lechner, Wien. 
Besonderes ultraviolettempfindliches Papier, das leider nur kurze Zeit haltbar ist, wurde von $\mathrm{Ch}$. Schall ${ }^{1}$ ) mit $\mathrm{p}$-Toluidendiamin, p-Phenylendiamin u. a. hergestellt.

Für die Palimpsest- und forensische Photographie wird man gewöhnlich zu diesen außerordentlichen Hilfsmitteln nicht greifen müssen und sich mit der photomechanischen Platte begnügen können.

Ein Vergleich von Aufnahmen mit Strahlen von $366 \mu \mu$ und von $313 \mu \mu$ läßt erkennen, daß der Gegensatz zwischen Schrift und Unterlage mit der Wellenlänge abnimmt, und daß bei Strahlen unterhalb $290 \mu \mu^{2}$ ) die Reflexionsfähigkeit der Schrift im Vergleich zum Planum mitunter zunimmt, was für forensische Aufnahmen moderner Papiere und Schriften ausgewertet werden kann. Für Aufnahmen mit Strahlen von weniger als $\lambda=253 \mu \mu$ wäre daher eine Lichtquelle ${ }^{3}$ ), wie sie von Lenard und Ramsauer für andere Zwecke gebaut wurde, sehr wertvoll.

Mit ultravioletten Strahlen können öfters verschiedenartige wertvolle, unerwartete Aufschlüsse über einen Palimpsest gewonnen werden. So hat der Verfasser einst mit sichtbarem Licht nach dem älteren Verfahren einen Palimpsest (Historia Langobardorum Pauli Diaconi, Municipalbibliothek Assisi) aufgenommen. Auf einem der Negative wurden Umrisse einer Zeichnung erhalten, die deutlich eine männliche Gestalt in mittelalterlicher Kleidung darzustellen schien. Dies war nicht auffallend, da der Palimpsest noch andere radierte Zeichnungen enthielt. Eine Ultraviolettaufnahme gab aber sofort Aufschluß, daß die Gestalt seltsamerweise durch Umrisse eines Wasserfleckens od. dgl. gebildet war.

I) Ztsch. f. wiss. Photographie, I9II, S. 89. Eders Jahrbuch, 1908, S. 442 ; 1909. S. $25^{2}$; 1910, S. 439. Ed er, Handbuch, Bd. 3 , S. 330.

2) Mit prismatisch zerlegtem Ultraviolett.

3) Über die Wirkungen sehr kurzwelligen ultravioletten Lichtes auf Gase usw. Sitzungsbericht d. Heidelberger Akademie, Math.naturw. Klasse, I910, Abhandlung 28. 
Über die Verwendung der ultravioletten Strahlen für forensische und ähnliche Untersuchungen sind, wie früher schon erwähnt, auch von anderer Seite einige Veröffentlichungen erfolgt ${ }^{1}$ ).

\section{Die Wiedergabe des Palimpsestes durch Fluoreszenz (nicht reflektierte Strahlen) bei ultravioletter Beleuchtung.}

Aus den bisherigen Ausführungen geht hervor, daß alle Möglichkeiten der Wiedergabe des Originals durch das von ihm reflektierte Licht, sei es sichtbar oder unsichtbar, erschöpft sind.

Photographische Vergleichsaufnahmen von Palimpsesten, die, in vergangenen Jahrzehnten teilweise mit Reagenzien behandelt, den wirklichen Schriftbestand zeigten, teilweise aber in ihrem ursprünglichen Zustand erhalten blieben, ließen erkennen, daß ausgedehnte Schriftgebiete sich der Differenzierung mit den vorher beschriebenen Verfahren entzogen hatten.

Erwägungen rein theoretischer Natur über die Möglichkeit einer unterscheidenden Abbildung zweier gleichfarbiger Objekte waren als Richtschnur für weitere Versuche in feste Fassung zu bringen. Abgesehen von den technischen Mitteln und praktischen Ausführung erschien es als das einfachste, aber auch einzig Mögliche, zwei Körper von gleicher Strahlenreflexionsfähigkeit, aber verschiedener chemischer Beschaffenheit photographisch dadurch zu differenzieren, daß der eine selbstleuchtend gemacht würde, während" der andere dunkel bliebe.

Bekanntlich fluoreszieren nun viele organische Stoffe bei ultravioletter Beleuchtung. Es wurde daher im dunkeln Raum

I) Photographie mit unsichtbaren Strahlen. Von O. Mente. Photogr. Rundschau, I9r2, Heft 7, S. ro3. Die ultravioletten Strahlen in der Photographie. Von O. Mente. Ztsch. f. Reproduktionstechnik,

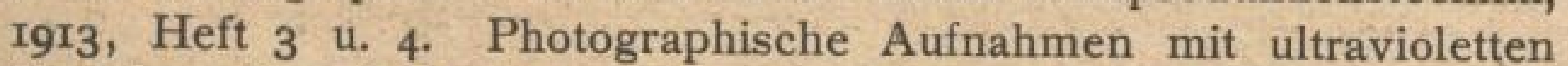
Strahlen. Von J. Richter. Umschau, Bd. 18, S. 628, I914. 
untersucht, ob das Pergament fluoreszieren, der Schriftkörper aber vorwiegend dunkel bleiben würde. Dies trifft zu.

Hier beginnt erst die eigentliche Palimpsestphotographie mit einer wesentlich neuen Grundlage, der Fluoreszenz ${ }^{1}$ ). Wie die Fluoreszenz erzeugt wird, ist und bleibt gleich. Die Erzengung der Fluoreszenz durch ultraviolette Strahlen ist entsprechend dem Stand der heutigen Technik die einfachste, aber nur durch die Zeit bedingt. Für den Nichtfachkundigen sei besonders hervorgehuben, daß das Wesentliche der neuen Palimpsestphotographie nicht in einer Entdeckung von ultravioletten Strahlen, die schon seit mehr als einem Jahrhundert (J. W. Ritter, 1801) bekannt sind, liegt, noch in der Erzeugung solcher Strahlen, sondern in der Hervorrufung einer Schrift und Planum unterscheidenden Fluoreszenz mit irgendeinem geeigneten Mittel und deren technischen Verwertung.

Durch die Fluoreszenz wird das Pergament zum Selbstleuchter. Die einzelnen Bestandteilchen, die in unmittelbarer Nähe aneinanderliegen, beleuchten sich gegenseitig, ebenso die unteren Teile auch die oberèn. Dadurch wird das Pergament teilweise zu einem beleuchteten Objekt. Notwendigerweise entsteht ein kleiner Verlust an Schrift. Theoretisch ließe er sich mit den physikalischen Mitteln beheben, die die Fluoreszenzstrahlen in einer zur Leuchtfläche senkrechten Lage bringen bzw. in die Richtung zum Brennpunkt des Objektivs. Dadurch würde eine scheinbare Helligkeitsverminderung eintreten, die Schriftausbeute aber auf das theoretische Maximum gebracht werden.

\section{- Die Erzeugung der Fluoreszenz durch spektrales Ultraviolett im allgemeinen ${ }^{2}$ ).}

Bekanntlich wird ein schmaler Streifen polychromen Lichtes durch ein Prisma in seine Bestandteile zerlegt. Es entsteht ein Spektrum mit einer Farbenordnung, in der sich immer

I) D. R. P. ${ }^{288} 3^{27}$ (Inhaber: P. R. Kögel).

2) Mit der Ultraviolett-Projektionseinrichtung der Firma C. Zei ßs, Jena. 
kürzere bzw. längere Wellen aneinanderreihen, so daß sich an Infrarot Rot, Gelb, Grün, Blau, Violett, Ultraviolett anschließen.

Zur Herstellung eines umfangreichen Spektrums werden die Strahlen der Lichtquelle (Abb. 6) (a) durch einen Kollektor $(b)$ gesammelt und auf einen Spalt $(d)$ geführt. Das aus dem Spalt austretende Licht wird durch den Kondensor $(t)$ auf ein Prisma $(e)$ geleitet. Es entsteht bereits ein Spektrum, das durch ein zweites, nebenanstehendes Prisma $(s)$ noch weiter zerlegt und dann auf die Vorlage $(r-u)$ geworfen wird. Das Spektrum besteht aus nebeneinanderliegenden monochromatischen Bildern des Spaltes.

\section{Die Lichtquelle und Einzelnbestandteile der ultravioletten Projektionseinrichtung.}

Für stationäre Anlagen empfiehlt sich nur die Quarzlampe ${ }^{1}$ ) (Abb. 7), da sie kèinerlei Wartung bedarf und stets gebrauchsfertig ist. Sie besteht aus einem Leuchtrohr mit zwei Polgefäßen (- Kathode, + Anode), die beidé Quecksilber enthalten. Die Einführungselektroden bestehen meist aus einer Metallegierung, wie Nickelstahl, dessen Ausdehnungskoeffizient mit dem des Quarzes so weit übereinstimmt, daß die Elektroden beim Erwärmen die Einschmelzstellen der Quarzröhre nicht sprengen. Zur Verteilung der Wärme sind die beiden Polgefäße mit fächerarfigen Radiatoren versehen. Die Zündung der Lampe kann handtätig oder automatisch erfolgen.

Die Vorrichtung für automatische Kippung sei kurz beschrieben (Abb. 8). Der Brenner $a$ wird an einer quer über

I) Die Quarzlampe. Von Dr. J. C. Pohle, Berlin Igr4. Über eine neue Quarzlampe berichtet W. Wolfke, Zürich. Verein d. Deutsch. physik. Gesellsch., Bd. 19, S. 239, 1917. Da ich die Originalabhandlung bis zur Stunde nicht einsehen konnte, gebe ich ein kurzes Referat aus der Zisch. f, Elektrotechnik u. Maschinenbau XXXVI, Heft 8, S. 99, wieder: „Die neue Lampe besitzt im Gegensatz zu den bekannten mit schwach geneigtem, horizontalem Leuchtrohr ein kreisförmig ausgebildetes, vertikales Leuchtrohr, was für die meist vertikale Spalte der optischen Versuche bequemer ist." 
dem Leuchtrohr liegenden Metallachse $h$ in zwei Lagern getragen; durch die Zugstange $K$ ist er mit dem Solenoidkern $i$ der Nebenschlußspule $g$ verbunden Beim Einschalten des Stromes wird die Spule $g$ magnetisiert, die den Kern $i$ in die Höhe hebt. Dadurch wird das positive Polgefäß durch die Zugstange gehoben, das negative gesenkt, so daß von einem zum andern Quecksilber überläuft, eine stromleitende Verbindung hergestellt und der Hauptstromkreis geschlossen wird. Der Hauptstromkreis magnetisiert die Drosselspule $d$, die den Anker $c$ anzieht und dadurch den Nebenstromkreis bei $b$ unterbricht. Der Brenner fällt in seine Anfangslage zurück, das Quecksilber trennt sich und der Lichtbogen entsteht. Die Drosselspule $d$ dient nicht nur dazu, den Nebenschlußstrom nach dem Zünden der Lampe zu unterbrechen, sondern auch, um als Selbstinduktanz ein plötzliches Abfallen der Spannung auszugleichen. An den Vorschaltwiderstand $e$, der durch einen auf Porzellanhülsen aufgewundenen Eisendraht besteht, wird der' Strom mittels des Gleitkontaktes $f$ auf die richtige Volt spannung eingestellt. Die Eisenwiderstände haben die Anfgabe, die hohe Stromstärke während des Anlassens der Lampe in -mäßigen Grenzen zu halten. Der Anfangsstrom erhitzt den Draht sehr stark (bis zur schwachen Rotglut), wodurch sein Widerstand sich um das Vierfache erhöht, was für die eingebrannte Lampe die günstigste Stromzufuhr ergibt.

Unmittelbar nach dem Zünden ist die Voltspannung sehr gering, die Stromstärke an Ampere dagegen sehr groß. Allmählich steigt die Voltspannung, während die verbrauchte Amperemenge abnimmt. Nach etwa 20 Minuten ist ein andauernder Zustand erreicht. Die Temperatur der Dampfsäule beträgt in ihren verschiedenen Schichten einige tausend Grade und der Dampfdruck erreicht etwa eine Atmosphäre.

Mit zunehmendem Dampfdruck (und Wattverbrauch) nimmt die Intensität des ultravioletten Spektrums im allgemeinen zu, und zwar schneller als die des sichtbaren Spektrums. 
Über die Zunahme der ultravioletten Strahlen aus dem Wattverbrauch gibt folgende Tabelle ${ }^{-1}$ ) Aufschluß:

\begin{tabular}{|c|c|c|c|}
\hline \multicolumn{3}{|c|}{ Belastung der $\mathrm{Hg}$-Lampe } & \multirow{2}{*}{$\begin{array}{l}\text { Intensitat } \\
\text { der ultravioletten } \\
\text { Strahlung }\end{array}$} \\
\hline Volt & Ampere & Watt & \\
\hline $3^{6}$ & 2,03 & $73, \mathbf{I}$ & 28,2 \\
\hline $3^{6}$ & 2,23 & 80,5 & 29,8 \\
\hline 37 & $2,3 I$ & 85,3 & 30,2 \\
\hline $3^{8}$ & 2,40 & $9 \mathrm{r}, 3$ & 30,3 \\
\hline 39,5 & 2,50 & 98,7 & 30,7 \\
\hline 42 & 2,66 & III, 5 & $3 I, 8$ \\
\hline 44 & 2,73 & 120,5 & $3 I, 8$ \\
\hline 46 & 2,79 & 128,5 & 32,1 \\
\hline $4^{8}$ & 2,86 & $\mathbf{I} 37,0$ & $3^{2,4}$ \\
\hline $5 I, 5$ & $2,9 I$ & I 50,0 & $\begin{array}{r}33,9 \\
+\end{array}$ \\
\hline 56 & 2,90 & 162 & $3^{6}$ \\
\hline $\begin{array}{l}01 \\
65\end{array}$ & 2,90 & $\begin{array}{l}\text { I77 } \\
\text { I }\end{array}$ & 39,2 \\
\hline 69 & 2,92 & 202 & 46,8 \\
\hline 72 & 2,92 & 210 & 50,5 \\
\hline 75,5 & 2,93 & 222 & 55,0 \\
\hline 79 & $\begin{array}{l}2,95 \\
2,84\end{array}$ & 233 & 59,9 \\
\hline $\begin{array}{l}04 \\
77\end{array}$ & $\begin{array}{l}2,8 I \\
2,94\end{array}$ & $\begin{array}{l}179,5 \\
227\end{array}$ & $\begin{array}{l}40,2 \\
55,8\end{array}$ \\
\hline 89 & 2,93 & $26 I$ & $\begin{array}{l}55,0 \\
71,4\end{array}$ \\
\hline 100 & 3,03 & 303 & 99,0 \\
\hline IIO & 3,03 & 337 & 126,5 \\
\hline 125 & 3,06 & $3^{82}$ & $\mathrm{I}_{7} 6$ \\
\hline I35 & 3,04 & 4IO & $2 I 4,5$ \\
\hline I 49 & 3,12 & 465 & 294 \\
\hline 162 & 3,12 & 505 & 353 \\
\hline 172 & 3,13 & $53^{8}$ & 390 \\
\hline
\end{tabular}

Man wäre geneigt, diesen Zahlen entsprechend anzunehmen, daß eine bestimmte Quarzlampe mit größerem Wattverbrauch auch größere Menge ultravioletter Strahlen geben müßte, wodurch dann die Beleuchtung der Vorlage kürzer gehalten werden könnte. In welchem $\mathrm{Ma} B$ eine solche Regel bei einem bestimmten Brennertypus zutrifft, konnte bisher nicht ganz sicher bestimmt werden.

Der Verfasser arbeitete längere Zeit mit einer Quarzlampe für 220 Volt Gleichstrom und $1^{1} / 2$ Ampere (Brennerspannung

I) Photometrische und spektralphotometrische Messungen am Quecksilberbogen bei hohem Dampfdruck. Von Dr. Küch und T. Retschinsky. Ann. d. Physik, Bd. 20, Heft 3, S. $56_{3}$ ff., Igo6. 
160 Volt), später an einem anderen Ort mit einer Quarzlampe für 110 Volt und $2 \frac{1}{2}$ Ampere (Brennerspannung $80 \mathrm{Volt}$ ).

Die Expositionen mit der ersten Lampe mußten etwa doppelt solange gebalten werden als mit der zweiten. Da die Voltspannung am ersten Benutzungsort stets zu gering und unregelmäßig war, so lag es nahe, die geringe Intensität der ultravioletten Strahlung diesem Umstand zuzuschreiben, und vielleicht mit Recht.

Es ist aber noch ein anderer Umstand ins Auge zu fassen. Die Dicke des Leuchtfadens ist bei 220 Volt infolge des in dem Leuchtrohr herrschenden höheren Dampfdruckes geringer als bei 110 Volt. Der Spalt der Projektionsvorrichtung bestimmt nun den wirksamen Umfang des Leuchtfadens. Dieser ist nun bei gleicher Spaltöffnung bei 220 Volt geringer als bei 110 Volt.

Im allgemeinen hat aber $\mathrm{Lux}^{1}$ ) gezeigt, daß bei einem bestimmten Brenner die aktinische Wirkung der Strablen, an der Ultraviolett stark beteiligt ist, mit dem Wattverbrauch nicht proportinal ist, und daß eine Lampe nach der Einbrennperiode bei konstantem, niederem Stromverbrauch das Maximum an "Hefner-Kerzen" gibt, die aktinische Wirkung aber weit hinter der zurückbleibt, die bei geringerem Stromverbrauch (Watt) früher vorbanden war.

Tabelle von Lux:

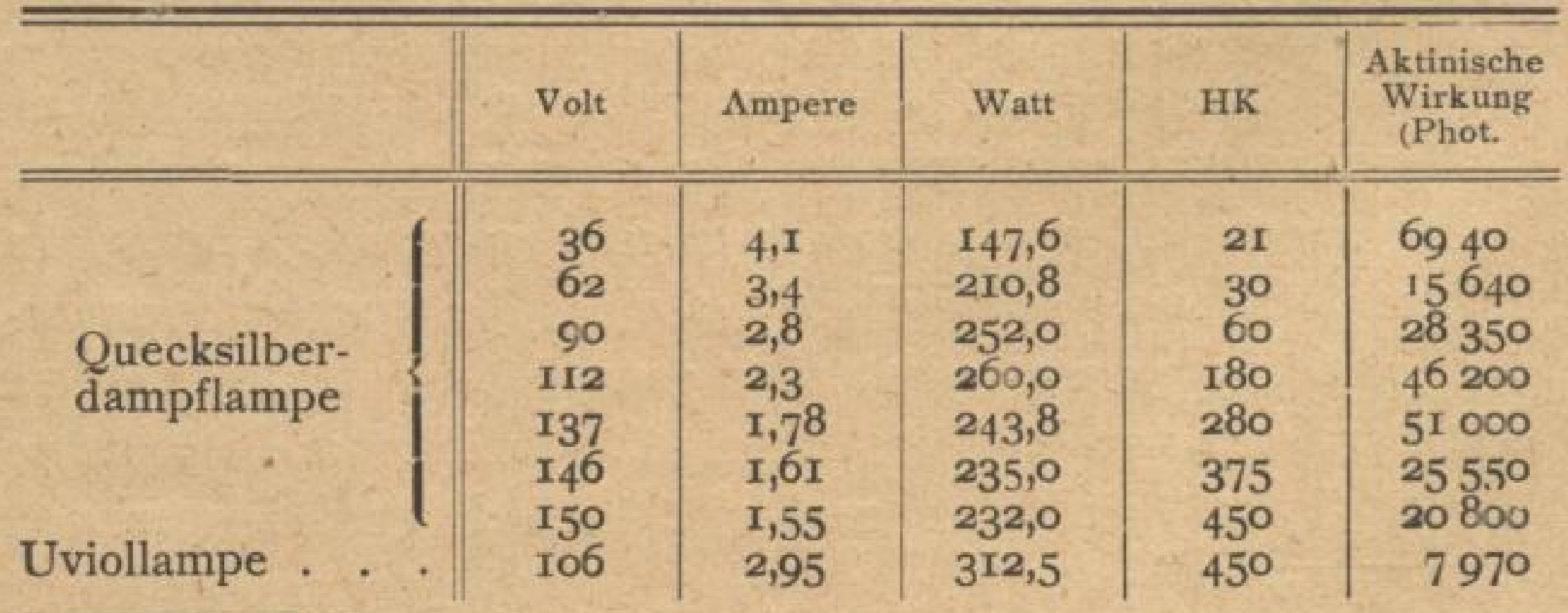

I) Die künstlichen Lichtquellen in der Photographie. Ztsch.f. Beleuchtungswesen usw., S. 55 ff., I9I5. 
Bemerkenswert sind folgende Zahlen:

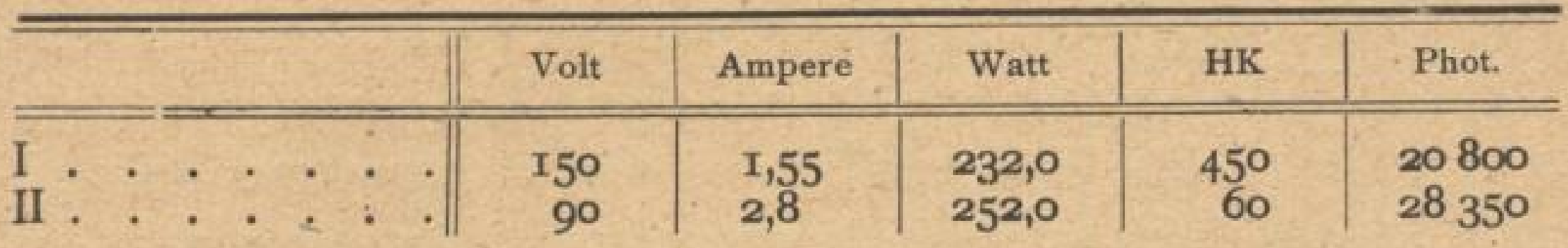

Bei einem Verbrauch von 232 Watt ist die Lichtstärke (HK) viel größer ałs bei dem Verbrauch von 252 Watt. Fall I würde annähernd der Lampe ${ }^{1}$ ) für 220 Volt (Brennerspannung 160 Volt) und 1,5 Ampere entsprechen, Fall II der Lampe ${ }^{1}$ ) für 110 Volt (Brennerspannung 80) und 2,5 Ampere.

Diese Zahlen würden also die Regel bestätigen, daß die aktinische Wirkung mit dem Wattverbrauch zunehmen müsse.

Zwei andere Zahlen der gleichen Tabelle besagen aber das Gegenteil:

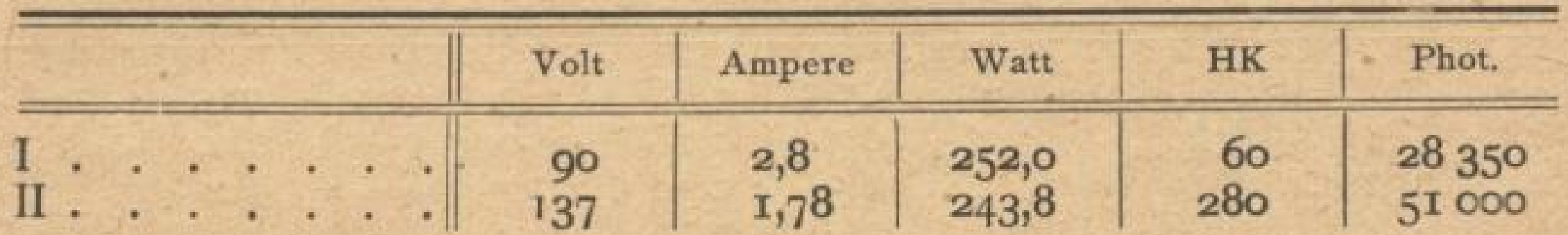

Mit geringerem Wattverbrauch wird die maximale aktinisehe Wirkung erreicht.

Daraus geht zum wenigsten das hervor, daß es unzulässig ist, ohne empirischen Beweis anzunehmen, daß eine Lampe mit höherem Wattverbrauch stets größere Mengen ultravioletter Strahlen liefert. Es ist vielmehr anzunehmen, daß der Bau des Brenners, die Temperatur, der Dampfdruck und das Potentialgefälle verschieden zusammenwirken.

Es ist daher nicht unerwartet, wenn auch von anderer Seite bekannt wurde, daß nicht alle Quarzlampen, die für gleichen Volt- und Ampereverbrauch gebaut werden, gleiche Mengen an ultravioletten Strahlen lieferten. So berichtet Winter ${ }^{2}$ ):

I) Geliefert durch die Quarzlampengesellschaft, Hanau a. M.

2) Über die Strahlung der Quarzquecksilberlampen. Von Ch. Winter. Aus dem chemischen Universitätslaboratorium in Kopenhagen. Ztsch. f. Elektrochemie, Bd. 20, Nr, 24, S. Iog. 
„Vor kurzem hatte ich Gelegenheit, zwei Quarzquecksilberlampen für 110 Volt und 3,5 Ampere, eine Heraeus- und eine Cooper-Hewitt-Lampe zu vergleichen. Die Energiemessungen wurden mit Hilfe des von mir früher beschriebenen Fluorometers ausgeführt und sind, wegen der Kürze der zur Verfügung stehenden Zeit, nicht als Präzisionsmessungen aufzufassen. Die Energiewerte E, die in willkürlicher Einheit ausgedrückt sind, lassen sich für die beiden Lampen direkt vergleichen."

Heraeus-Lampe.

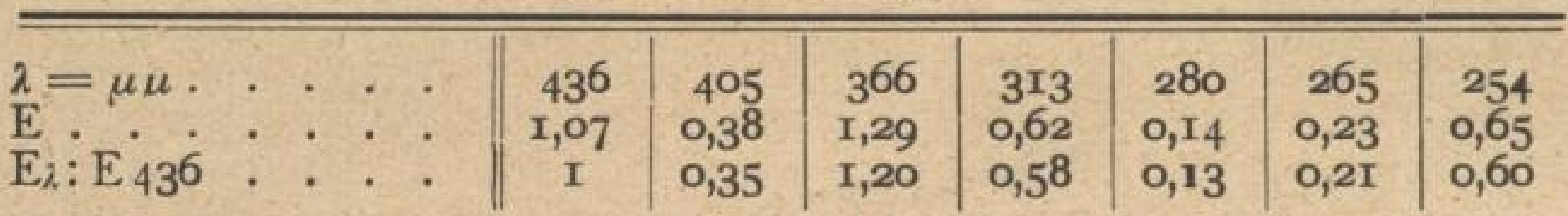

Cooper-Hewitt-Lampe.

\begin{tabular}{|c|c|c|c|c|c|c|c|c|c|}
\hline $\mathrm{E}_{436}$ & : & $\dot{0}$ & $\begin{array}{r}436 \\
0,36 \\
1 .\end{array}$ & $\begin{array}{l}405 \\
0,11 \\
0,31\end{array}$ & & $\begin{array}{l}313 \\
0,10 \\
0,31\end{array}$ & $\frac{280}{-}$ & $\begin{array}{l}265 \\
0,007 \\
0,02\end{array}$ & $\begin{array}{c}254 \\
0,01 \\
0,04\end{array}$ \\
\hline
\end{tabular}

"Ein Vergleich der Energiewerte zeigt erstens, daß die blaue, violette und ultraviolette Strahlung der Cooper-HewittLampe erheblich schwächer als diejenige der Heraeus-Lampe ist, und zweitens, daß das Übergewicht der letzteren Lampe desto stärker hervortritt, je kleiner die betrachteten Wellenlängen sind. Es wird dies durch folgende Zusammenstellung besonders deutlich hervorgehoben."

\begin{tabular}{|c|c|c|c|c|c|}
\hline $\begin{array}{l}\text { E Cooper-Hewitt : Heraeus } \\
\lambda=\mu \mu\end{array}$ & $\begin{array}{r}0,34 \\
436\end{array}$ & $\begin{array}{r}0,29 \\
405\end{array}$ & $\begin{array}{r}0,14 \\
366\end{array}$ & $\begin{array}{l}0,16 \\
3^{1} 3\end{array}$ & $\begin{array}{l}0,03 \\
265\end{array}$ \\
\hline
\end{tabular}

Über die Abnahme der ultravioletten Strahlen mit dem Altern der Lampen berichten Bordier ${ }^{1}$ ), Courmont und Nogier ${ }^{2}$ ); dem widerspricht Henri ${ }^{3}$ ).

1) Bordier, Archives d'electricité medicale, S. 396, I9ı.

2) J. Courmont und Ch. Nogier, Compt. rend., Bd. 152, S. 1746 , 1911 .

3) V. Henri, Compt. rend., Bd. I53, S. 428 , I9rI. 
In neueren Zeiten wurden sowohl von Heraeus als von der Cooper-Hewitt \& Cie. Quecksilberlampen für Wechselstrom gebaut. Eine solche Lampe besitzt drei Polgefäße. Die Wechselstromlampen sind, abgesehen von dem getrennten Transformator und der fakultativen, automatischen Zündvorrichtung, für alle Netzspannungen gleichgebaut, im Gegensatz zu den Gleichstromlampen, die für verschiedene Netzspannungen verschieden gebaut sind ${ }^{1}$ ). Von der Cooper-Hewitt-Wechselstromlampe wird von Girard berichtet, daß sie weniger gut $\operatorname{sei}^{2}$ ).

Beim Erwerb von Quarzlampen für Gleichstrom empfehlen sich der Billigkeit wegen die kleineren Typen für 1,5 Ampere und 200-240 Volt oder für 2,5 Ampere und 100-140 Volt. (Metalfa-Lampen der Quarzlampengesellschaft Hanau a. M.) Die größeren Typen für 4-6 Ampere besitzen ein größeres Ultraviolettemissionsvermögen und weisen bei relativ geringem Wattverbrauch eine größere Lichtausbeute auf. Stärkere Lampen sind aber nur von bedingtem Werte.

Die Fluoreszenz des Pergamentes, wie die anderer organischer Verbindungen, streben nämlich eiflem Maximum zu, das auch mit stärkerer Beleuchtung nicht gesteigert werden kann.

Das Pergament, das an sich schon nur einen Teil der ultravioletten Strahlen in Fluoreszenzstrahlen umwandelt und den übrigen Teil reflektiert, wird mit der zunehmenden Stärke der Lichtquelle auch mehr ultraviolette Strahlen reflektieren, die entweder durch das Glas des Objektives oder die flüssigen ultravioletten Absorptionsfilter zurückgehalten werden müssen. Denn in der Fluoreszenzphotographie dürfen keine ultravioletten Strablen auf die Platte gelangen. Das ultraviolette Absorptionsvermögen dieser Filter ist nun relativ. Sehr groß̉e Mengen langwelliger ultravioletter Strablen, zu denen wir hier die von

I) Die neue Wechselstrom-Quecksilberlampe. Von Ingenieur F. Girard, Elektrotechn. Zeitschr., Bd. 33, S. 676, 1912.

2) Über eine neue automatische Quecksilberlampe für Wechselstrom. Von J. C.Pole. Elektrotechn. Zeitschr., Bd. 3I, S. 929, 1910. 
$\lambda=366 \mu \mu$ zählen wollen, werden von dem Flüssigkeitsfilter nicht mehr ganz absorbiert. Starke Lichtquellen bieten hier also keinen Vorteil, sie können eher schaden.

Für direkte ultraviolette Photographie sind sie dagegen von Nutzen. Denn der Gegensatz im Bild nimmt mit der. Intensität der Beleuchtung zu. •

Der weitere Aufbau der ultravioletten Projektionseinrichtung gestaltet sich im einzelnen folgendermaßen. Der Brenner ist in einem lichtdichten Gehäuse (Abb. 9) eingeschlossen, so daß Strahlen nur dürch den in das Gehäuse hineinragenden Kollektor $b$ nach außen gelangen können. Der Kollektor soll sich in einem Abstand von etwa $1 \mathrm{~cm}$ vom Brenner befinden.

Die Strablen gelangen dann auf einen in nächster Nähe stehenden Spalt $c$, dessen Öffnung regulierbar ist. Die Lichtschneiden des Spaltes dürfen nicht geradlinig wie bei Spektroskopen sein, sondern etwas gebogen, so daß sie zwei parallele Kreisausschnitte darstellen (Abb. 10). Wären die Lichtschneiden des Spaltes nämlich geradlinig, so würden die Spaltbilder auf der Vorlage gebogen sein. Dies wäre aber sehr nachteilig. Die Pergamente besisen die Grundform rechteckiger Parallelogramme, und die ultravioletten Bänder können nur dann in ihrer ganzen Breite ohne unnütze Ausdehnung verwertet w̌erden, wenn sie die gleiche Form wie die Vorlage besitzen.

Durch die Schrauben $a$ a kann der Spalt geöffnet und geschlossen werden. Die Millimeterskala erlaubt, das jeweilige Öffnungsverhältnis genau festzustellen. Wird der Spalt eng gehalten, so werden die Linien des Spektrums schmal und deutlich. Zwischen den Linien finden sich dunkle Zonen. Wird der Spalt geöffnet, indem beide Lichtschneiden mit Hilfe der Schrauben a a gleichmäßig voneinander entfernt werden, so verschwinden die dunklen Zonen, die Spaltbilder breiten sich nach beiden Seiten aus und gehen allmäblich ineinander über.

Welche Ausdehnung den ultravioletten Bändern für die Palimpsestphotographie gegeben werden darf, wird später näher angegeben. 
Zum Scharfeinstellen der Linie auf der Vorlage, d. h. zunächst auf einem Phosphoreszenz- oder Fluoreszenzschirm, wird der Spalt eng gehalten. Der Kondensor mit einer Brennweite von ungefähr $16 \mathrm{~cm}$ wird auf der optischen Bank in einem Abstand von ungefähr $16 \mathrm{~cm}$ zum Spalt aufgestellt. Die Prismen bzw. das Prismentischchen folgt dem Kondensor $t$ in kurzem Abstand (Abb. 9).

Wenn die Linien des sichtbaren Spektrums auf dem Phosphoreszenzschirm scharf erscheinen sollen, wird der Kondensor auf der optischen Bank den Prismen genähert, sollen die ultravioletten Linien scharf erscheinen, so wird der Kondensor etwas gegen den Spalt geschoben. Die Quarzlinse als Monochromat hat für die Strahlen verschiedener Wellenlänge verschiedene Brennpunkte.

Das durch den Kondensor auf ein erstes Prisma geleitete Licht wird durch ein nahestehendes zweites Prisma weiter abgelenkt und so in die einzelnen Bestandteile eines Spektrums zerlegt.

Die Prismen (Abb. 11) sind so aufgestellt, daß die Austrittskathete $a$ des ersten Prisma $e$ mit der Eintrittskathete $a^{\prime}$ des zweiten Prisma $s$ einen Winkel von etwa $105^{\circ}$ bildet.

Wenn die Prismen in dieser Stellung auf dem Tisch (Abb. 1 l) feststehen, dieser aber um die Achse $l$ gedrebt wird, so verschiebt sich das Spektrum auf dem Phosphoreszenzschirm zunächst in gleicher Richtung, kehrt aber beim weiteren Drehen des Tischchens um. Die Stellung des Tischchens, die das Spektrum unbeweglich zeigt, gibt das Minimum der Ablenkung. Die Linien des Spektrums sind dann gleichmäßig und zeigen keine „Fahnen".

\section{Der Phosphoreszenz- und Fluoreszenzschirm.}

Um das Feld der unsichtbaren, auffallenden Strahlen finden zu können, wird das Spektrum auf einen Phosphoreszenz- oder Fluoreszenzschirm entworfen. Auf diesem werden die ultravioletten Linien durch Erregung von gelbgrünem Lumineszenzlicht erkannt. 
Der Phosphoreszenzschirm wird durch Auftragen von Sid otBlende $^{1}$ ) (Schwefelzink) auf ein Leinen hergestellt. Die durch die Einwirkung der ultravioletten Strahlen phosphoreszierenden Strahlen leuchten auf dem Schirm noch nach, wenn bereits kein Licht mehr auf diesen einwirkt. Wenn das Spektrum daher verschoben werden soll, so ist mit einer Neubeleuchtung zu warten, bis die Phosphoreszenz abgeklungen ist, damit keine Vermischung der alten und neuen leuchtenden Linien und Irrtum in der richtigen Auswahl der Linien vermieden wird.

Ein Fluoreszenzschirm ${ }^{2}$ ) zeigt diesen Nachteil nicht Er leuchtet etwas schwächer und hört sofort mit der Lichtwirkung auf zu lumineszieren. Ein solcher Schirm wird hergestellt, indem man weißes, saugfähiges Papier (Löschpapier) oder Leinwand mit einer wässerigen Lösung von Chininsulfat oder Fluoreszeinnatrium, oder zur Not mit Petroleum oder einem alten, braungewordenen Fixierbad tränkt. Ein solcher Schirm hält monatelang. Er empfiehlt sich besonders für Arbeiten auf Reisen, da er sehr schnell am Gebrauchsort (und billig) hergestellt werden kann. Er läßt sich rollen und falten, was beim Phósphoreszenzschirm nicht möglich ist, da seine Leuchtfarbe beim Biegen- leicht abbröckelt.

Der Phosphoreszenz- oder Fluoreszenzschirm wird senkrecht zur optischen Achse der Kamera aufgestellt, wie die Vorlage in Abb. 1, s. Die optische Achse der Kamera und der Beleuchtungsvorrichtung (bis zu den Prismen) müssen, nach rückwärts ausgezogen, einen Winkel von etwa $45^{\circ}$ bilden.

\section{Das Spektrum.}

Das Emissionsspektrum des Quecksilbers zeigt folgende Linien. In Abb. 12 ist $r$ orangegelb von $579 \mu \mu$ und $576 \mu \mu$,

I) Die Selbstherstellung von luminiszierenden Leuchtplatten. Von Dr. F. Novak, Eders Jahrbuch für Photographie und Reproduktionstechnik, S. 430 , I9I4. - Leuchtende Papiere usw. Von L. E. Andès, S. 109, Wien 1896.

2) Vgl: Druckschrift Mikro $321, Z$ eiß, Jena. 
breit, in Wirklichkeit gemäß der natürlichen Ausdehnung beim Scharfeinstellen auf den mittleren Teil des ultravioletten Spektrums ${ }^{1}$ ). Die starke Linie $g$ ist hellgrün von $546 \mu \mu$. Dunkelgrün und schwach ist $d g$ von $492 \mu \mu$. Es folgt die kräftige blaue Linie $b$ von $436 \mu \mu$. Schwächer und auf dem Leuchtschirm durch gelbgrünes Phosphoreszenzlicht aufgehellt ist Violett, $v$, von $405 \mu \mu$. Die erste, intensive ultraviolette Linie ist $u$ von $366 \mu \mu$. Es folgt eine schwache Linie $i$ von $334 \mu \mu$. Schmal, aber kräftig, ist die nächste Linie $k$ von $313 \mu \mu$. Nicht so stark sind die nebenan befindlichen Linien von $302 \mu \mu$ und $296 \mu \mu, m$ und $n$. Es folgt dann die sehr schwache Linie von $292 \mu \mu$. Ziemlich stark ist die Linie $t$ von $289 \mu \mu$. Es folgen noch die Linien von 275, 270, $265,253 \mu \mu$. In der Fluoreszenz-Palimpsestphotographie werden fast ausnahmslos die Linien von $366 \mu \mu$ und $313 \mu \mu$ benutzt.

Zur Ausdehnung der Linien zu breiten Bändern wird der Spalt so weit geöffnet, daß die Linie $u$ von $366 \mu \mu$ die entsprechende Breite von $G-D$, oder daß die Linie $k$ von $313 \mu \mu$ den proportionalen Raum von $E-R$ einnimmt.

Bei der Aufnahme mit Strablen von $313 \mu \mu(E-R)$ ist streng zu beachten, daß sie nicht mit solchen von $366 \mu \mu$ $(G-D)$ vermischt werden, außer es wird vor das Objektiv ein Ultraviolettabsorptionsfilter gebracht, das die Strahlen von $366 \mu \mu$ hindert, durch das Objektiv auf die Platte in der Kamera zu gelangen, wodurch das Fluoreszenzbild daselbst zu verschleiert würde. Die Linien $m, n, k$ und $i$ können ohne Bedenken in das Band $E-R$ eingezogen werden, da die vom Glas des Objektives gleich den Strahlen von $313 \mu \mu$ der Hauptmenge nach absorbiert werden.

Mit der Entfernung des Schirmes oder der Vorlage ron der Beleuchtungsvorrichtung nimmt bei gleichbleibender Spaltbreite die Ausdehnung der Bänder zu. Die Beleuchtungskraft, die der beleuchtenden Fläche umgekehrt proportional ist, nimmt

1) Die Buchstaben der Abb. I2 sind nicht identisch mit den Fraunh of erschen Linien. 
mit der Ausdehnung des Bandes ab. Die Expositionen sind dementsprechend zu verlängern.

Die Intensität der einzelnen Linien ist verschieden. Die wichtigsten Linien im sichtbaren und unsichtbaren Emissionsspektrum einer Quarzlampe bei 85 Volt Lampenspannung und 2 Ampere Strom besitzen folgende Energiewerte:

Wellenlänge

$579 \mu \mu$ und $576 \mu \mu, \mathrm{Gelb}$. . . . . . 13,

$546 \mu \mu$, Grün . . . . . . . . . . 19,

$436 \mu \mu$, Blau . . . . . . . . . . . 5,

$405 \mu \mu$, Violett . . . . . . . . . . 2,5

$366 \mu \mu$, Gruppe Ultraviolett . . . . . . 9,

$313 \mu \mu$, " $\quad$. . . . . . . 6,

$253 \mu \mu, \quad " \quad, \quad$. . . . . . . 1 . 1 .

Die Linie von $366 \mu \mu$ ist also die stärkste im ultravioletten Spektrum.

Auf das Original darf während der Exposition kein sichtbares Licht gelangen, da es das Fluoreszenzlicht überdeckt und dann nur mehr eine gewöhnliche Photographie liefert. Der Arbeitsraum muß daher streng dunkel gehalten werden. Sichtbares Nebenlicht, sowie der sichtbare Teil des Spektrums werden auf ziveckmäßige Weise abgeblendet und abgefangen, wie in Abb. $6(h)$ und Abb, 13 angegeben ist.

In Abb. 13 ist $r$ ein Tisch, der die Ultraviolett-Projektionsvorrichtung trägt. Auf dem Tisch $\approx$ steht die Vorrichtung $n$ (in Abb. 6 mit $h$ bezeichnet), mit der das sichtbare Spektrum abgefangen wird. In Abb. 13 sind $t$ verschiebbare Starkpapiere, die aus der Öffnung, $e$ nur die in Verwendung kommenden ultravioletten Strahlen austreten lassen.

\section{Die Erzeugung der Fluoreszenz mit spektralem Ultraviolett von $313 \mu \mu$ und mit Silberfilter.}

Das Original muß den Strahlen frei exponiert werden. Es kann also nicht mit einer Platte aus gewöhnlichem Glas glattgehalten werden, da dieses die ultravioletten Strahlen von 
$313 \mu \mu$ infolge Absorption überhaupt nicht zum Original gelangen lassen würde. Dies wäre nur mit einer dünnen Platte aus weißem Uviolglas ${ }^{1}$ ) möglich.

Das Original wird in Ermanglung einer solchen Platte seitlich festgehalten, was am besten gelingt, wenn man dasselbe an seinen äußeren Rändern in einem sogenannten Spindelkopierrahmen gegen zwei Leisten vorsichtig drückt (Abb. $1 s$, Abb. 14 u. 15).

Der in Abb. 14 u. 15 dargestellte, von P. Jans O.S. B. entworfene Spindelrahmen zeichnet sich durch den Vorteil aus, daß gebrechliche Codices nur halb geöffnet und so auf das beste geschont werden. Die Handhabung des Rahmens geht aus den beiden Darstellungen leicht hervor.

Das Original wird dann in das Band $E-K$ gebracht.

Die Fluoreszenzaufnahme mit $313 \mu \mu$ kann auch auf eine andere Weise als mit spektralem Ultraviolett ausgeführt werden, und zwar mit Hilfe des früher besprochenen Quarzsilberfilters. Die Beleuchtungsvorrichtung erhält zu diesem Zwecke eine andere Anordnung. Unmittelbar an das äußere Rohrende des Kollektors wird auf einem Träger das Quarzsilberfilter (wie in Abb. 5 die Blauuviolglasküvette) angebracht. Auf der optischen Bank folgt der Kondensor (Abb. 1, c). Durch entsprechendes Verschieben derselben, d. h. durch Einstellung auf den Brennpunkt, wird dadureh auf dem Leuchtschirm eine homogene, kreisrunde, - am Rand scharf begrenzte Lumineszenzscheibe erzeugt. Weist diese innere Unregelmäßigkeiten auf; so ist gewöhnlich der Kollektor etwas zu weit vom Brenner der Quarzlampe entfernt.

Mit dem Silberfilter ist es möglich, sehr große Vorlagen gleichmäßig zu beleuchten. Bei einem Abstand von $2-3 \mathrm{~m}$

I) Der Verfasser hat die wenigen großen Platten aus weißem Uviolglas von Schott u. Gen., Jena, aufgekauft. Nach mündlicher Mitteilung des Fabrikvertreters ist eine Neuherstellung solcher Platten wegen den großen technischen Schwierigkeiten nicht mehr zu erwarten. 
zwischen der Vorlage und der Beleuchtungsvorrichtung kann leicht eine Fluoreszenzscheibe von $1 / 2 \mathrm{~m}$ Durchmesser und mehr erzielt werden.

Gegenüber der Beleuchtung mit spektralem Ultraviolett hat dieses Verfahren den (wenn auch nicht sehr großen) Nachteil, daß die Expositionen etwas länger gehalten werden müssen, da das Silberfilter selbst einen Teil der ultravioletten Strahlen zurückhält.

Für die Trennung der Strahlen, die unterhalb $\lambda=313 \mu \mu$ liegen, kann man ein Bromdampffilter benutzen, das A. Wiegand nach einem Vorschlag von $\mathrm{Cornu}$ für ärophysikalische Forschungen verwendet hat. Das Bromdampffilter absorbiert die Strahlen von Gelbgrün bis etwa zur Linie von $\lambda=303 \mu \mu$. Von dort an bis zum Gebiete von $\lambda=265 \mu \mu$ wird das Filter vollkommen durchlässig. Wiegand benutzte ein Glasrohr von $20 \mathrm{~cm}$ Länge und $3,5 \mathrm{~cm}$ Weite, dessen beide Enden mit einer Quarzplatte und einer plankonvexen Quarzlinse abgeschlossen wurden. Durch ein seitliches Ansatzrohr wurde so viel flüssiges Brom eingegossen, daß im Innern der Röhre der Sättigungszustand des Bromdampfes erhalten blieb. Flüssiges Brom in dünner Schicht oder auch Bromwasser sind nicht brauchbar, da sie die Schärfe der Absorptionslinien herabsetzen ${ }^{1}$ ).

\section{Die Erzeugung der Fluoreszenz mit spektralem Ultraviolett von $366 \mu \mu$ und dem Blauuviolfilter.}

Das Original wird in das Band $G-H$ gebracht. Unebene Pergamente oder solche, deren Schrift bis an den äußersten Rand gehen und daher seitlich nicht festgehalten werden können, werden mit einer Platte von gewöhnlichem Glas, die aber nicht mehr als etwa $2 \mathrm{~mm}$ dick sein soll, glattgehalten.

Ein Teil des auffallenden Ultraviolett wird von der Vorlage zurückgeworfen und muß auf seinem Weg zur Platte auf-

I) Physik. Ztsch., Bd. I4, S. I444, I9r3. Ztsch. f. wissenschaftl. Photographie, Bd. 18, S. 237. 
gehalten werden, damit es das Fluoreszenzbild nicht verschleiert Dies geschieht mit einem Ultraviolett-Absorptionsfilter ${ }^{1}$ ), das unmittelbar vor das Objektiv gebracht wird (Abb. $1 a$ u. Abb. 4).

Dieses Ultraviolett-Absorptionsfilter besteht z. B. aus einer Glasküvette, die mit einer Lösung von $0,5 \mathrm{~g}$ anthrachinonsulfosaurem Natron in $100 \mathrm{cbm}$ Wasser gefüllt wird. Der innere Abstand der Küvettenwände und daher der Flüssigkeitsschicht soll $5 \mathrm{~mm}$ betragen.

Die Beleuchtung des Originals mit Strahlen von $366 \mu \mu$ kann auch mit dem Blauuviolglasfilter erfolgen (Abb. 5). Die eine Kammer $(a)$ der Küvette wird mit einer Lösung von $25 \mathrm{~g}$ Kupfersulfat in $100 \mathrm{cbm}$ Wasser $^{2}$ ) gefüllt. Für die andere Kammer wird $0,1 \mathrm{~g}$ Nitrosodimethylanilin in $100 \mathrm{cbm}$ absolutem Alkohol gelöst, wovon zum Gebrauch je $2 \mathrm{cbm}$ der Vorratslösung mit je $10 \mathrm{cbm}$ Wasser gemischt werden. Es ist Sorge zu tragen, daß im oberen Teil der Küvette keine Luftblasen vorhanden sind, durch die sichtbares Licht auf die Vorlage gelangt. Wenn also nach längerer Zeit ein Teil der Filterflüssigkeit durch Erwärmen infolge der nahen Stellung der Küvette an der Lichtquelle verdunstet, muß aufgefüllt werden. Nach 6 Stunden wird die gesamte Nitrosodimethyllösung durch eine neue ersetzt, da der Farbstoff allmählich ausbleicht und dann sichtbare Strahlen durchläßt.

Die Verbindung des Blauuviolfilters mit der Lichtquelle in einer möglichst raumgeringen Vorrichtung ist in der Zeißschen Ultraviolett-Filterlampe durchgeführt (Abb. 1, $u$ ). Die Lampe kann mit einem entsprechenden Widerstand in Gleichund Wechselstrom angeschlossen werden. Die positive Nickeldochtkohle liegt in der optischen Achse. Die negative Kohle (Marke Noris) steht vertikal. Die positive Kohle ist dünner als die negative. Eine Verwechslung der beiden ist aber ausgeschlossen, weil die Klemmfassung, für die Kohle entsprechend

I) Weiteres im Kapitel 5 .

2) Die Kupfersulfatlösung wird vor Gebrauch jedesmal filtriert. Koge1, Die Palimpsestphotographie. 
dimensioniert ist. Die Lampe wird mit der Hand reguliert. An dem lichtdichten Gehäuse sitzt ein ausziehbarer Tubus, der zwei Quarzkollektorlinsen von $40 \mathrm{~mm}$ Öffnung enthält. Im äußeren Ende des Tubus befindet sich die Blaunviolglaskürette. Sie wird von der Firma C. Zeiß in zweifacher Form ausgeführt: Mit einer Flüssigkeitskammer allein, die mit 20 prozentiger Kupfersulfatlösung zu füllen ist. Das Gelbfilter ist trocken und ist zwischen den Wänden der Küvette eingeschlossen. Da jedoch zu befürchten ist, daß das Gelbfilter bei längerem Gebrauch ausbleicht und durch seine Einkittung zwischen den Glaswänden nicht ohne weiteres ausgewechselt werden kann, so ist die Doppelküvette mit zwei Flüssigkeitskammern vorzuziehen. Die der Lichtquelle zunächstgelegene Kammer wird mit der 20 prozentigen Kupfersulfatlösung gefüllt, die andere Kammer mit einer Nitrosodimethylanilinlösung. Zu diesem Zwecke werden $0,1 \mathrm{~g}$ Nitrnsodimethylanilin in $100 \mathrm{ccm}$ absoluten Alkohol gelöst. Von dieser Vorratslösung werden $2 \mathrm{ccm}$ mit $10 \mathrm{ccm}$ Wasser gemischt.

Diese Lampe empfiehlt sich vor allem für Arbeiten auf Reisen, da sie, wie gesagt, mit entsprechendem Widerstand überall an dèn elektrischen Strom angeschlossen werden kann.

Die Aufstullung der Lampe mit dem Kondensor, der eine Brennweite von $16 \mathrm{~cm}$ bei einer Öffnung von $40 \mathrm{~mm}$ hat und der zu einer gleichmäßigen Verteilung des Lichtes unumgänglich notwendig ist, ist in Abb. 1 (c) dargestellt. Man kann aber auch die billigeren Linsen aus U.V.-Krone-Glas benutzen, die Zeiß mit einer Brennweite von $200 \mathrm{~mm}$ liefert. Der Widerstand $n$ ist für eine Spannung von 110 Volt und einen Stromverbrauch von 5 Ampere.

Etwas billiger und noch einfacher in der Ausführung sind die Ultraviolett-Filterlampen der Firma Leitz in Wetzlar und der Optischen Werkstätten Reichert in Wien. Die LeitzLampe enthält anstatt des Quarzkollektors, wie ihn die ZeißLampe besitzt, einen solchen aus weißem Uviolglas, das ultrasiolette Strahlen größerer Wellenlänge leichter durchläßt als 
gewöhnliches Glas. Für ultraviolette Strahlen kürzerer Wellenlänge ist es aber bei weitem nicht so durchlässig als Quarz, sehr kurze läßt es überhaupt nicht mehr durch. Die Strahlen des Nickelkohlenbogenlichts werden durch das weiße Uviolglas aber in großen Mengen durchgelassen.

Für forensische Untersuchungen und zur Aufnahme besonders stark radierter Palimpseste ist die Verwendung der Strahlen von $366 \mu \mu$ weniger empfehlenswert. Man bediene sich der Verfahren mit Strablen von $313 \mu \mu$ und noch kürzerer ${ }^{1}$ ). Die ultravioletten Strahlen größerer Wellenlänge vermögen nämlich noch schwache Tintenreste zu durchdringen und erregen die Fasern, die die Tinte tragen, zur Fluoreszenz. Sie wirken dann durch ihr Strahlen auf der lichtempfindlichen Platte wie das. Planum, von dem sie sich durch Nichtleuchten unterscheiden sollten.

\section{Das Objektiv.}

Die geringe Lichtstärke des Fluoreszenzbildes verlangt ein Objektiv, das bei voller Öffnung das Original in Naturgröße scharf wiedergibt. Es muß also sphärisch, anastigmatisch, und chromatisch wenigstens für die blauen und gelben Strahlen, korrigiert sein. Die Brennweite soll etwa ein Drittel länger sein als die Diagonale des auszuzeichnenden Formates. Man wird im allgemeinen guttun, immer ein solches Objektiv zu erwerben, das das nächstgrößere Format scharf und ohne Blende auszeichnet, als in den Preislisten des Handels empfohlen wird.

Das Öffnungsverhältnis zur Brennweite bestimmt die Lichtstärke eines Objektives. Für die Palimpsestphotographie sind nur Objektive ${ }^{2}$ ) mit $\mathrm{F}: 4,5-6,8 \mathrm{zu}$ benutzen.

Seltene, sehr große Vorlagen, die vom Objektiv ohne Blende nicht scharf abgebildet werden, wird man vorteilhaft

I) Siehe Hinweis auf die Lenard-Ramsauersche Lichtquelle S. r6. Ihre großen Anschaffungs- und Betriebskosten werden leider einer allgemeinen technischen Anwendung hinderlich im Wege stehen.

2) Tessar von Zeiß-Jena, Celor von Goerz-Berlin u. a. 
zuerst verkleinert (ohne Blende) aufnehmen und dann nach Bedarf mit gewöhnlichen optischen Mitteln vergrößern.

In der forensischen Anwendung der Fluoreszenzphotographie werden die früher erwähnten Mikroobjektive benutzt. Das Mikroobjektiv soll ohne Blende eine $15-20$ fache Vergrößerung mit hinreichender Bildschärfe geben.

Ultraviolett-Absorptionsküvetten wird man hier nicht in den Strahlengang zwischen Objekt und Platte bringen, um die Bildschärfe nicht zu beeinträchtigen. Zur Beleuchtung des Objekts wählt man daher zweckmäßig nur spektrales Ultraviolett von $313 \mu \mu$.

Die Erzeugung der Fluoreszenz mit einem Silberfilter ist nur dann zulässig, wenn es keine Poren aufweist. Diese sind im dunkeln Raum zu erkennen, wenn man das Filter gegen starkes Licht hält. Durch solche Poren gelangen sichtbareStrahlen auf das Original, täuschen eine kräftige Fluoreszenz vor, geben aber nur eine gewöhnliche Photographie. Ein sokleines Loch kann die ganze Mikroaufnahme entwerten.

\section{Die lichtempfindliche Platte.}

Die gleìchen Gründe, die ein sehr lichtstarkes Objektiv verlangen, fordern auch eine höehstempfindliche Platte ${ }^{1}$ ) von 19-21 Scheinergrade.

Die äußerst zulässige Exposition dauert für solche Platten mit einer Quarzlampe für Gleichstrom von 110 Volt und 2,5 Ampere und einem Objektiv von $\mathrm{F}: 4,5$, bei Beleuchtung eines Palimpsestes im Format von $13 \times 18 \mathrm{~cm}$ :

mit spektralem Ultraviolett von $313 \mu \mu \quad 1$ Stunde, "Quarzsilberfilter $(313 \mu \mu)$. . . 2 Stunden,

" spektralem Ultraviolett von $365 \mu \mu \quad 2$ "

" der Blauuviolglasküvette $(365 \mu \mu) \quad 2$ "

Bei Benutzung der Zeißschen Ultraviolett-Filterlampe mit Nickelkohlen für Gleichstrom von 110 Volt und 5 Ampere und

I) Ultrarapidplatte von J. Hauff, Feuerbach in W., E. Lomberg in Langenberg (Rheinland), und von A. Lainer, Wien VII. 
bei Glatthalten des Originals mit einer $2 \mathrm{~mm}$ dicken Scheibe von gewöhnlichem Glas $1 / 2$ Stunde.

Stark gelbe und pigmentierte Originale oder solche, die mit der blauen „Giobertitinktur" behandelt wurden, verlangen eine längèe Belichtung.

Die weitere Behandlung der Platte erfolgt nach Gebrauchsanweisung der Fabrik.

Das gewonnene Negativ wird man nicht selten noch verstärken. W. Faworski ${ }^{1}$ ) empfiehlt besonders das Ozobromverfahren. W. Urban ${ }^{2}$ ) berichtet darüber, daß es gemäß seinen Versuchen bei zweimaliger Anwendung in den silberärmsten Negativpartien eine Deckung gibt, wie sie mit keiner der sonst im Trockenplattenprozeß üblichen Verstärkungsmethoden - nicht einmal mit Uranverstärkung - auch nur annähernd erzielt wird. - Nach meinen persönlichen Erfahrungen wirkt es in der Tat gut.

\section{Das Ultraviolett-Absorptionsfilter ${ }^{3}$ ).}

Eine gedrängte Begründung für die Wahl der Bestandteile der Absorptionsfilter erscheint hier angezeigt.

Die ultraviolette Fluoreszenz wurde ron Stark zuerst beim Benzol entdeckt. Es zeigte sich dabei, wie bei anderen organischen Substanzen, daß die Fluoreszenz von einer Absorption begleitet ist. Eine Regel, die jedoch nicht ohne Ausnahmen ist, besagt, daß das absorbierte Licht von kürzerer Wellenlänge ist als das der ausgestrahlten Fluoreszenz. Dem Intensitätsmaximum des Fluoreszenzlichtes entspricht gewöhnlich ein Maximum in der Absorptionskurve.

Absorption und Fluoreszenz stehen im engen Zusammenhang mit der chemischen Konstitution des Körpers. Es zeigte

I) Die Anwendung des Ozobromverfahrens zur Wiederherstellung zerstörter Schriften. Von W. Faworski, Photogr. Rundschau S. $27,1912$.

2) Beiträge zur Praxis der gerichtlichen Photographie. Von W. Urban, Eders Jahrbuch, S. 276, 1912.

3) Siehe Literaturangaben am Schluß dieses Kapitels. 
sich, daß mit zunehmender Kondensation des Benzols, das der Grundstoff der zyklischen Kohlenwasserstoffverbindungen ist, die Absorptions- und die Fluoreszenzbänder gegen das sichtbare Gebiet des Spektrums verschoben werden.

Doppelten und dreifachen Kern des Benzols $\left(\mathrm{C}_{6} \mathrm{H}_{6}\right)$ besitzen Naphthalin $\left(\mathrm{C}_{10} \mathrm{H}_{8}\right)$ und Anthrazen $\left(\mathrm{C}_{14} \mathrm{H}_{10}\right)$.

Benzol zeigt 7 Absorptionsbänder von $233-271 \mu \mu$ und

4 Fluoreszenzbänder von $267-310 \mu \mu$,

Naphthalin zeigt 4 Absorptionsbänder von $242-320 \mu \mu$

und 9 Fluoreszenzbänder von $314-357 \mu \mu$,

Anthrazen zeigt 4 Absorptionsbänder von $320-380 \mu \mu$

und 4 Fluoreszenzbänder von $380-450 \mu \mu$.

Das Benzol besitzt Homologen, in denen an die Phenylgruppe $\left(\mathrm{C}_{6} \mathrm{H}_{5}\right)$ ein oder mehrere Alkyle $\left(\mathrm{CH}_{3}\right)$ gelagert sind. Das erste Homologe des Benzols ist Toluol $\left(\mathrm{C}_{6} \mathrm{H}_{5} \cdot \mathrm{CH}_{3}\right)$, das nächste Xylol $\left(\mathrm{C}_{6} \mathrm{H}_{4}\left(\mathrm{CH}_{3}\right)_{2}\right)$ usw.

Mit der Einführung eines Alkyls in den Benzolring steigert sich allgemein die Stärke der Fluoreszenz, das Absorptionsband wird breiter und verschiebt sich nach Rot.

Das Xylol kommt in drei isomeren Verbindungen vor, in denen die Zahl der Kohlen- und Wasserstoffe die gleiche bleibt, während die Anordnung im Molekel eine andere ist. Diese isomeren Disubstitutionsprodukte werden als Ortho-, Meta- und Paraverbindungen unterschieden.

Nach Sauer und Mies nimmt die Absorption in folgender Ordnung zu: Ortho-, Meta-, Paraverbindung.

Ein Isomeres des Anthrazen ist Phenanthren. Nach Stark und Steubing zeigt es aber geschwächt die Anthrazenbanden.

Höhere molekulare Kohlenwasserstoffe mit kondensiertem Benzolkern sind Reten $\left(\mathrm{C}_{18} \mathrm{H}_{20}\right)$, Chrysen $\left(\mathrm{C}_{18} \mathrm{H}_{12}\right)$.

Die Absorption und Fluoreszenz der Kohlenwasserstoffverbindungen werden durch solche Substitutionsprodukte, die einen sichtbaren Färbkörper bilden, sehr stark beeinflußt.

Für die Palimpsestphotographie $m u ß$ das Ultraviolett- $A b-$ sorptionsfilter farblos sein, da sichtbare Farbstoffe einen Teil 
des Fluoreszenzbildes, das aus blauen, gelben und roten Strahlen besteht, absorbieren würden.

Die Wahl des Anthrazens als Hauptbestandteil eines der Ultraviolett-Absorptionsfilter für die Strahlen von $366 \mu \mu$ wurde durch sein Absorptionsvermögen für das gesamte Gebiet von $320-380 \mu \mu$ bestimmt.

Anthrazen (depurat subl. Merck) ist ein leicht gelbes kristallinisches Pulver, das in geringen Mengen durch Benzol, Toluol, Xylol u. a. wasserklar gelöst wird.

Zur Lösung des Anthrazens wird im vorliegenden Fall aber Phenetol $\left(\mathrm{C}_{6} \mathrm{H}_{5} \mathrm{OC}_{2} \mathrm{H}_{5}\right)$, ein Äther des Phenols benutzt. Es besitzt seine Absorptionsmaxima bei etwa 360 und $380 \mu \mu$. Der Grund der Wahl des Phenetols war aber nicht sein Absorptionsvermögen für $366 \mu \mu$, sondern sein photochemisches Verhalten mit dem Anthrazen.

Das Anthrazen geht nämlich bei langer Beleuchtung in Dianthrazen über, das in Benzol usw. unlöslich ist und als weißer Niederschlag ausfällt. Im Dunkeln verwandelt sich das Dianthrazen wieder in Anthrazen zurück. Der photochemische Vorgang ist in folgender Weise umkehrbar:

Licht

$$
\begin{gathered}
2 \mathrm{C}_{14} \mathrm{H}_{10} \rightleftarrows \mathrm{C}_{28} \mathrm{H}_{20} \text {. } \\
\text { Dunkel }
\end{gathered}
$$

Die Verwandlung des Anthrazens zu Dianthrazen geht im Licht bei verschiedenen Lösungsmitteln nicht gleich schnell vor sich, äußerst langsam im Phenetol, wie aus folgender Tabelle von Luther und Weigert erhellt:

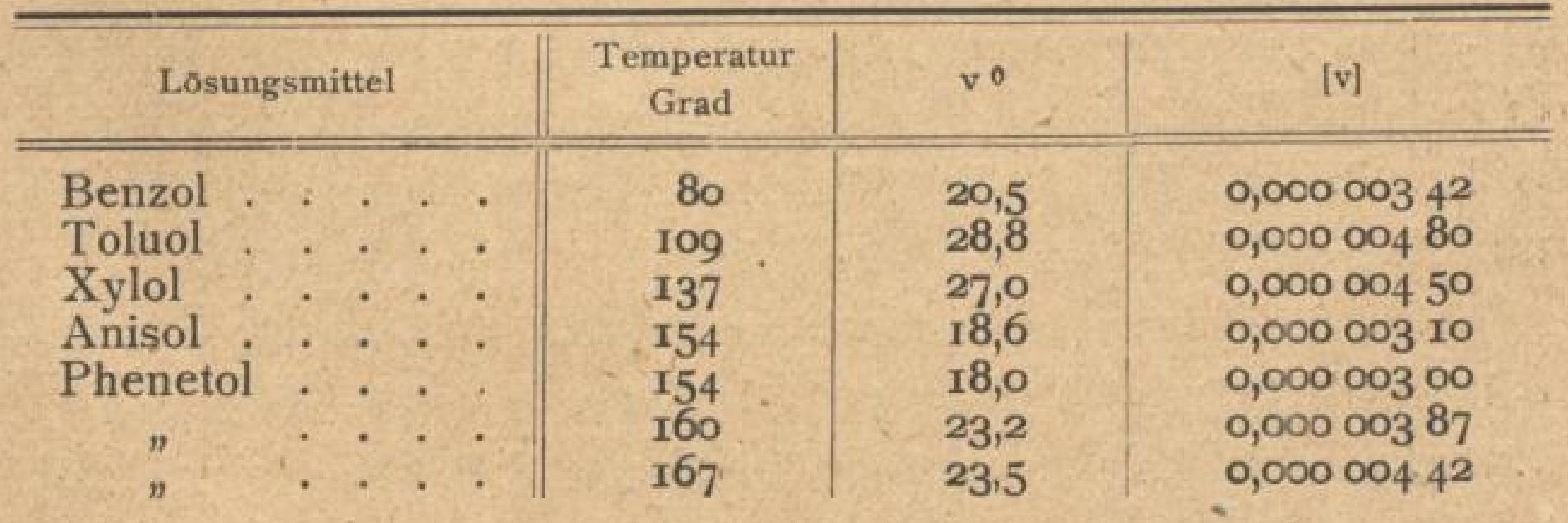




$$
\nabla^{0}=\text { Reaktionsgeschwindigkeit } \cdot[v]=\frac{\nabla^{0}}{60 \cdot 10^{6}} \text { die Menge }
$$

Anthrazen in Gramm-Molen, die in $1 \mathrm{ccm}$ der Lösung in der Sekunde verschwindet, wenn die bestrahlte Oberfläche dieses Kubikzentimeters $1 \mathrm{qcm}$ groß ist und sich in $1 \mathrm{~cm}$ Entfernung von der Lichtquelle befindet. Die Lichtabsorption wird als vollständig in der bestrahlten Schicht angerommen.

Die Bildung des Dianthrazens geht also im Phenetol bei $154^{0}$ am langsamsten vor sich und fällt mit der Temperatur, wie der Vergleich der Zahlen $154^{\circ}$ und $18,0 \nabla^{0}$ mit den Zahlen $167^{\circ}$ und $23,5 \nabla^{0}$ zeigt.

Bei Zimmertemperatur verläuft die Reaktion äußerst langsam. Wenn daher das Filter außer Gebrauch im Dunkeln aufbewahrt wird, was nachts gewöhnlich mehrere Stunden der Fall sein wird, so braucht das Filter kaum erneuert zu werden. Falls das Anthrazen kristallinisch ausfällt, genügt Lösen durch Erwärmen.

Die Filterlösung muß gut luftdicht abgeschlossen sein, da das Phenetol verdunstet und das Anthrazen bei seinem Übergang in Dianthrazen durch den Sauerstoff der Luft verharzt und gelb wird.

Ein anderes Ultraviolett-Absorptionsfilter besteht aus einer Lösung von $0,5 \mathrm{~g}$ Triphenylmethan in $70 \mathrm{ccm}$ absolutem Alkohol. Das Triphenylmethan ist teurer als Anthrazen. Dafür bietet es aber den sehr schätzenswerten Vorteil, daß die Substanz kalt gelöst werden kann und somit auch nicht ausfällt, was beim Anthrazen leicht der Fall ist. Das Absorptionsvermögen dieser Substanz erklärt sich leicht aus dem Vorhergesagten, denn Triphenylmethan besteht aus drei Benzolkernen, die an die Methingruppe gebunden sind, und besitzt die Formel $\mathrm{C}_{6} \mathrm{H}_{5} \cdot \mathrm{CH} \cdot\left(\mathrm{C}_{6} \mathrm{H}_{5}\right)_{2}$. Der Verfasser benutzt gegenwärtig fast nur dieses Filter.

Ein anderes Absorptionsfilter besteht aus $0,5 \mathrm{~g} \beta$-anthrachinonsulfosaurem Natron und $100 \mathrm{ccm}$ Wasser. Auch diese Lösung kann kalt hergestellt werden. Sie weist eine leicht 
gelbe Farbe auf, was sich jedoch bei der photographischen Aufnahme praktisch kaum bemerkbar macht, solange die Lösung frisch ist. Durch Licht wird sie braun. Auch hier erklärt sich das Absorptionsvermögen leicht aus der Konstitution der Substanz $\mathrm{C}_{6} \mathrm{H}_{4}(\mathrm{CO})_{2} \mathrm{C}_{6} \mathrm{H}_{8} \mathrm{SO}_{3} \mathrm{Na}$.

Es ist klar, daß auch andere Substanzen, die den Benzolkern zwei- oder dreimal enthalten, sich für den genannten Zweck mehr oder weniger eignen. So besitzen z. B. Terpene von der Zusammensetzung $\mathrm{C}_{15} \mathrm{H}_{24}$ ein ziemlich hohes $\mathrm{Ab}$ sorptionsvermögen für Ultraviolett und übertreffen in dieser Hinsicht die Terpene der Zusammensetzung von $\mathrm{C}_{10} \mathrm{H}_{10}$, die ihrerseits dem Benzol zurückstehen müssen. Dagegen vermag nicht jede "Gelbscheibe", die vom Handel geliefert wird und die Violett und Blau in erheblichem Maße absorbiert, die ultravioletten Strahlen von $\lambda=366 \mu \mu$ in gleichen Mengen zurückhalten, wie das farblose Anthrazen oder Triphenylmethan. Dies wird man auch erwarten, wenn man beachtet, daß z. B. Nitrosodimethylanilin, ein ausgesprochen gelber Körper, geradezu ein Ultraviolettfilter ist, d. h. Ultrariolett durchläßt.

Zum Schluß noch einige Worte über die Fluoreszenz selbst.

Die Fluoreszenz beruht nicht auf einer chemischen Veränderung (auch beim Pergament nicht).

Der Kürze wegen sei die Angabe von H. Ley im Wörterbuch der Naturwissenschaften, Bd. 3, S. 1236, wiedergegeben: "Die Beobachtung, daß schon Stoffe von denkbar einfachster Konstitution, nämlich einatomige Gase, wie Quecksilherdampf u.a., fluoreszenzfähig sind, weist darauf hin, daß es sich bei der Erscheinung um einen inneratomistischen Vorgang handeln wird, und in der. Tat sind alle Versuche, die Fluoreszenz letzten Eudes auf Grund von chemischen Umlagerungen oder Tautomeriephänomenen zu erklären, ats gescheitert an. zusehen."

P. Lenard und J. Stark haben den Fluoreszenzerscheinungen eine elektroatomistische Auslegung gegeben. 


\section{$-42-$ \\ Literatur.}

Stark und Meyer, Beobachtungen über die Fluoreszenz der Benzolderivate, Physik. Ztsch., Bd. 8, S. 250, 1907.

W. E. Pauli, Über ultraviolette und ultrarote Phosphoreszenz. Ann. d. Physik, Bd. XXXIV, S. 755, r9rr.

Über die grundlegenden Arbeiten P. Lenards, über die Phosphoreszenz, siehe die Literaturangaben in Ztsch. f. physik. u. chem. Unterricht, Heft 3, 1916, S. 150. Von H. Schmid.

Umfangreiche Literaturangaben usw. über die Absorption anorganischer und organischer Stoffe enthält Bd. 3, Handbuch d. Spektroskopie, 1905. Von H. Kayser. - Über Triphenylylmethan siehe z. B. S. 572.

Über gelbe, ultraviolett durchlässige organische Substanzen siehe: P. KrüB, Über die Absorption organischer Farbstoffe im Ultraviolett (Ztsch. f. physik. Chemie, Bd. 10, 1905, S. 257). Eder und Valenta, Beiträge zur Photochemie und Spektralanalyse, III. Teil, Halle (Saale) rgo4.

The Relation between Absorption Spectra and Chemical Constitution. Part. XI. Some aromatic Hydrocarbons. Journal of Chem. Society Vol. XCIII, Part. II, 1908, p. 1902 . - By E. Baly \& W. Tuck. Part. XII. Some Aminoaldehydes and Ketons of Aromatic Series: Journal of the Chem. Society. Vol. XCIII, Part. II, 1908, p. 2108.

I Über die ultraviolette Fluoreszenz des Benzols und einiger seiner Derivate. Von E. Dickson. Ztsch. f. wissenschaftl. Photographie, Bd. X, S. 166 , 181 .

Das Absorptionsspektrum der drei Xylole im Ultraviolett. Von W. Mi es. Ztsch. f. wissenschaftl. Photographie, Bd. VIII, I9Io, S. 287 .

Über die ultraviolette Absorption des Benzols. Von L. Grebe. Ztsch. f. wissenschaftl. Photographie, Bd. IX, S. זзо.

Das Absorptionsspektrum des Tolvols im Ultravioletten. Von F. Cremer. Ztschr. f. wissenschaftl. Photographie, Bd. X, S. 349 .

H. Kauffmann, Die Beziehungen zwischen Fluoreszenz und chemischer Konstitution. I906.

Über umkehrbare photochemische Reaktionen im homogenen System. - Anthrazen und Dianthrazen. I. Robert Luther und Fritz Weigert. Ztsch. f. physik. Chemie, Bd. 51, 1905, S. 297. Abhandlung II in Bd. 53 , 1905, S. 385 . 


\section{6: Zusammenfassender Vergleich der Ausführungsmittel des Ultraviolett- Fluoreszenzverfahrens.}

Das Verfahren mit prismatisch zerlegtem Ultraviolett (Quarzlampe) ermöglicht Aufnahmen mit Strahlen von 366 und $313 \mu \mu$, und zwar ohne Lichtfilter zwischen Lichtquelle und Objekt. Bei der Beleuchtung mit Strahlen von $366 \mu \mu$ kann das Original mit einer dünnen Scheibe aus gewöhnlichem Glas glattgehalten werden. Das Ultraviolett-Absorptionsfilter ist vor dem Objektiv anzubringen.

Eine Beleuchtungsvorrichtung mit Silberfilter (also ohne Prismen) ist etwas billiger als eine Projektionsvorrichtung für spektrales Ultraviolett. Der Fluoreszenzkreis kann sehr umfangreich gestaltet werden. Ein Ultraviolettabsorptionsfilter ist entbehrlich. Das Glatthalten des Originals mit Glas ist nicht möglich.

Die Ultraviolett-Filterlampen und Blauuviolglasküvette ${ }^{1}$ ) mit Nickelkohlenbogenlicht gibt gleichfalls große Lumineszenzflächen. Das Original kann mit einer dünnen Glasscheibe glattgebalten werden. Vor das Objektiv ist das Ultraviolett-Absorptionsfilter zu bringen. Die Nitrosodimethylanilinlösung muß erneuert werden.

Bei der Zeiß-Lampe ist die obere Metalldochtkohle Anode (positiv) und nicht, wie bei anderen derartigen Lampen, Kathode (negativ). Die Zeiß-Lampe besitzt einen Kollektor aus Quarz, die Leitz-Lampe einen aus weißem Uviolglas. Beide Lampen können mit einem kleinen, regulierbaren Widerstand sowohl an Gleich - als Wechselstrom angeschlossen werden und eignen sich daher vortrefflich für Arbeiten auf wissenschaftlichen Reisen. Die Handhabung der Lampen ist sehr einfach (Abb. 16 u. 17).

I) Das Ultraviolettfilter und die Ultraviolett-Filterlampe als Apparate der Lumineszenzanalyse. Von Dr. H. Lehmann. Ztsch. f. Instrumentenkunde, I9I2, Heft 2, S. 43. 


\section{Die Schriftausbeute.}

Das Fluoreszenzverfahren gibt im Durchschnitt den vorhandenen Text der Palimpseste im Umfang von etwa $95 \%$ wieder. Die Ausnahmen bei Reagenzienflecken wurde bereits früher ${ }^{1}$ ) erwähnt.

Es kommt auch vor, daß das Pergament Tiefreliefe zeigt, die von ausgefallenen Buchstaben herstammen. Sie sind mit dem bloßen Auge leicht erkennbar und daher nicht der Gegenstand der eigentlichen Palimpsestphotographie. Neben solchen Reliefs finden sich oft wirkliche Schriftreste. Wurde nun ein solcher Palimpsest mit der bekannten Giobertitinktur behandelt, so wurde nicht selten der so entstandene Niederschlag mit dem Pinsel, der zum Auftragen des Reagens meist verwendet wird, in die Reliefs verschleppt. Es entstanden dann kräftige blaue Buchstaben. Dadurch wird der Schein erweckt, daß ein solches Reagens ${ }^{2}$ ) (abgesehen von der Verunstaltung des Originals) mehr Schrift zutage fördert als photographische Verfahren. Dieser Irrtum deckt sich sofort auf, wenn man bedenkt, daß schwache Tintenreste, die auf der Oberfläche liegen, nur zu leicht mit den Reagenzien verwischt werden.

Kräftige Reagenzien mit der notwendigen Zuziehung von starken Säuren, wie Salzsäure, zur Aufschließung der Eisenverbindungen würden rein technisch, abgesehen von anderen Übeln, nur dann einen Vorteil bieten, wenn die Schriftreste in einer unteren Schicht des Pergamentes liegen und die gesamte Oberfläche fluoresziert. Dieser Fall tritt in den einen Codices nie oder fast nie, in anderen in größerem Umfang auf. Er ist

I) Seite 6 .

2) Heutzutage ist die Anwendung von Reagenzien mit Recht allgemein verboten. Daßs sie aber heute dennoch angewandt werden, wenn das Verbot nicht besonders in den Bibliotheksverordnungen ausgedrückt ist, kann ich nachweisen. Vgl. auch: Hotz-Ostwald, Die Zerstörung der Codices und Palimpseste durch die modernen Gelehrten. Beiträge zur paläographischen Chemie und zur Geschichte der Technik. Ber. der Deutsch. Chem. Gesellschaft, S. I743, Jahrgang 1874 . 
von der Zusammensetzung der Tinte, die sich nach Zeit und Ort ändert, und durch die Kapilarität des Pergamentes bedingt.

Bemerkenswert ist ferner, daß Schwefelammonium, das heute noch mancherorts als ein den Palimpsesten unnachteiliges Reagens gilt, einen für das bloße Auge unsichtbaren Flecken erzeugt. Bei einer Beleuchtung mit Strahlen von $313 \mu \mu$ im dunklen Raum sieht man aber einen schwarzen Flecken, wie aus Tinte, im fluoreszierenden Planum, der die Schrift zudeckt. Bei Beleuchtung mit $366 \mu \mu$ leuchtet der Flecken manchmal, verliert aber gewöhnlich seine üblen Eigenschaften dadurch nicht, weil das Schwefelammonium über der Schrift liegt oder mit dieser eine chemische Verbindung eingegangen hat, die ebenfalls fluoresziert. Der Text ist daher mit dem in Kapitel 1 beschriebenen Verfahren aufzunehmen. Neues ist meist nicht mehr zu gewinnen ${ }^{1}$ ).

Wenn man den Grad der Radierung bei den Palimpsesten zum unterscheidenden Merkmal derselben wählt, kann man zwischen flachen und steilen Palimpsesten unterscheiden. Bei den flachen Palimpsesten wurde die Schrift auf einer großen Fläche, z. B. auf einer ganzen Seite gleichmäßig entfernt. Hierher gehören auch die Palimpseste, bei denen die Tinte durch Abwaschen und Bleichen getilgt wurde. Solche Palimpseste können dem bloßen Auge nur wenig Schrift erkennen und vermuten lassen. Wenn aber der Bestand der Schrift ein gewisses Minimum nicht unterschritten hat, liefern sie geradezu Schaustücke der Palimpsestphotographie.

In den steilen Palimpsesten blieb ein Teil der Schrift bestens erhalten, der andere wurde vollständig (mit dem Schabmesser) entfernt. Der Unkundige, der aber mit bloßem Auge noch sehr "viel" sieht, meint, \der übrige Teil müsse mit der Palimpsestphotographie sicher und glänzend zum Vorschein kommen. Wenn das Gegenteil eintrifft, ist er - mit Unrecht

I) Die blauen Giobertitinkturflecke und die roten von Rhodanammonverbindungen gestatten mehr Hoffnungen. Siehe S. 37 . 
- sehr erstaunt, wenn nicht enttäuscht. Der Sachkundige aber wird schon im voraus das Maß des $z^{\circ}$ Erwartenden ziemlich sicher bestimmen können.

An dieser Stelle soll auch noch der Rückwirkung moderner Konservierungsmittel für Handschriften (Gelatine, Cellit) auf das Ergebnis der Fluoreszenzphotographie gedacht werden. Aufnahmen von einem eigentlichen Palimpsest, der mit solchen Mitteln vor weiterem Zerfall gerettet werden sollte, liegen noch nicht vor. Soviel mir bekannt ist, wurde nur ein Palimpsest (Gaius, Verona) mit Gelatine behandelt.

P. F. Ehrle, S. J., der das Gelatinierverfahren ausgearbeitet hat, hat dem Verfasser zur Prüfung der Frage ein mit Seidenetz und Gelatine überzogenes Pergament übergeben. Das Seidenetz trat nicht sehr störend auf, da es in gleichem Maße wie Pergament fluoreszierte. Da die Gelatine, die hier in sehr dünner Schicht aufgetragen war, schwach fluoreszierte, traten starke Buchstaben noch gut hervor, während schwächere Schriftzüge zurückblieben. Bei eigentlichen Palimpsesten wäre ein Schriftverlust daher leicht erklärlich. Die Aufnahme würde man voraussichtlich am besten mit Strahlen von $366 \mu \mu$. Wellenlänge vornehmen, da diese Strablen die Gelatine noch durchdringen.

Versuche mit Cellit gaben wenig günstige Resultate. Es empfiehlt sich daher, zerbrechliche Palimpseste ohne jegliche Behandlung blattweise zwischen Glasscheiben aufzubewahren, wie es mit den Papyri üblich ist.

Die Zweckmäßigkeit des Fluoreszenzverfahrens zur Untersuchung forensischer Schriftstücke bedarf noch einer besonderen Besprechung.

Die zu hegenden Erwartungen hängen von der Natur des Papiers, der Tinte und der Art des Schreibens ab.

Das Papier besitzt kein so großes Aufsaugevermögen für die Tinte wie das Pergament. Bei diesem konnte von der Oberfläche der gesamte schwarze Tintenkörper entfernt werden, ohne daß dadurch die übrigen, nicht farbigen Eisenverbindungen und Gerbwirkungen in den unteren Schichten des Pergamentes 
vernichtet worden wären. Beim Papier sitzt der Tintenkörper jedoch fast nur auf der Oberfläche und kann daher leicbt entfernt werden. Oft bleiben im Papier aber Reste der eigentlichen Tinte oder von Tintenzusätzen $\left(\mathrm{H}_{2} \mathrm{SO}_{4}\right)$ und Veränderungen zurück, die unsichtbar, für eine Fluoreszenzaufnahme erfolgbringende Anhaltspunkte darstellen.

Im allgemeinen kann man sagen, daß gewöhnliche Schriftstücke, die bei einẹ Untersuchung mit älteren Verfahren absolut keinen Einblick in den Sachverhalt gewähren, auch durch die Fluoreszenzaufnahme meist keinen sicheren Aufschluß erwarten lassen. Wenn die älteren Verfahren aber geringe, wenn anch unzureichende Anhaltspunkte bieten, wird die. Fluoreszenzphotographie quantitativ und qualitativ Neues zutage fördern. Dauerexpositionen, die gut gedeckte Negative liefern, sind aber meist erforderlich (Abb. 26 u. 27). In sehr vielen Fällen wird die Fluoreszenzphotographie aber die letzte sichere Instanz zur Beurteilung des Untersuchungsobjektes bleiben.

Besonders günstig für die Fluoreszenzphotographie sind Flecken und Abdrücke von Fetten und Ölen. Sehr deutlich werden auch Papierfasern (von kaufmännischen, amtlichen und Wertpapieren) gegeben, und zwar meist auch dann, wenn mit den älteren Verfabren kein charakteristisches Merkmal gewonnen werden konnte (siehe Beispiele am Schluß).

Lumineszenzerscheinungen ${ }^{1}$ ), die bisher nur visuell, subjektiv beobachtet werden konnten, können nun auf photographischem Wege objektiv dargestellt werden. Bisher war

I) Die Ultraviolett-Filterlampe als wichtiges Hilfsmittel zur Bestimmung der Reinheit chemischer Produkte. Von O. Wolff. Chemiker-Ztg., Nr. 22, S. 197, 1912. Eine neue Methode zur Auffindung von Spermaspuren. Von R. Heller. Vierteljahrsch. f. gerichtl. Medizin u. öffentl. Sanitätswesen, Bd. 5o, S. 37-42. E. Engelhardt, Lumineszenzerscheinungen der Mineralien im ultravioletten Licht. Diss. Jena. Verhalten der Edelsteine und Perlen im ultravioletten Licht. Von L. v. Loehr. Sitzungsber. d.) Wiener mineralog. Ges., 4. März I9I2, S. I18. 
dies nicht möglich, denn zur Bestrahlung der Objekte wurden ebensolche ultravioletten Strahlen angewandt, die das Glas der Objektive mit größter Leichtigkeit durchdringen. Die ultravioletten Strahlen überdeckten das Fluoresżenzbild vollkommen. Die oben beschriebenen Ultraviolett-Absorptionsfilter lassen nun das Fluoreszenzbild allein wirksam werden und ermöglichen so die objektive Darstellung.

\section{Versuche der Erzeugung der Fluoreszenz mit subultravioletten und korpuskularen Strahlen ${ }^{1}$ ). Versuche mit den (subultravioletten) Röntgen- strahlen.}

Ein Durchleuchten von Palimpsesten mit X-Strahlen ließ nach Birgitti ${ }^{2}$ ) die Gegenwart von Zinnober, Mennige und Ultramarin erkennen. Schwache Tintenreste erzeugen jedoch wegen ungenügender Absorptionskraft kein Schattenbild.

Da seit den Versuchen Birgittis mehr als ein Jahrzehnt verflossen war und es unterdessen gelang, Röntgenröhren herzustellen, die sehr weiche X-Strahlen austreten lassen, mußte auf eine etwaige Fluoreszenzerregung des Pèrgamentes durch solche Strahlen geprüft werden.

Das Glas der gewöhnlichen Röntgenröhren, durch das die $\mathrm{X}$-Strahlen ins Freie treten, besteht der Hauptsache nach aus Natrium (Atomzahl 11), Kalzium (Atomzahl 20), Silizium (Atomzahl 14). Die Durchlässigkeit eines Elementes für $X$-Strablen ist eine Funktion der Atomzahl und nicht, wie früher angenommen, eine Funktion des Atomgewichtes. Die Absorptions-

I) Die Röntgenstrahlen sind sogenannte Ätherstrahlen von geringer Wellenlänge. Die Kathoden - und Anodenstrahlen sind korpuskulare Strahlen.

2) Birgitti, Bessarione, Vol. 6, r899 u. I900, P.216. La paleografia ed i raggi di Röntgen. 
fähigkeit eines zusammengesetzten Körpers entspricht der Summe der Absorptionsfähigkeit der Elemente der Molekel und nimmt in der vierten Potenz mit der Atomzahl zu.

Lindemann ${ }^{1}$ ). hat nun (wohl geleitet durch die frühere, annähernd richtige Regel) eine Röntgenröhre mit einem Fenster versehen, das sehr weiche $\mathrm{X}$-Strahlen durchläßt. Das Glas dieses Fensters besteht der Hauptsache nach aus Lithium (Atomzahl 3), Beryllium (Atomzahl 4), Bor (Atomzahl 5). Das Lindemannsche Glas besitzt keine sichtbare Eigenfluoreszenz.

Es wurde nun geprüft, ob die weichen, durch das Fenster austretenden $\mathrm{X}$-Strahlen Pergament zur Fluoreszenz erregen würden. Zum Abblenden des sichtbaren Lichtes wurde die Röhre hinter schwarzem Papier in Betrieb gesetzt. Das Vakuum wurde allmählich erniedrigt, so daß immer weichere Strahlen entstehen mußten. Die X-Strahlen, die durch schwarzes Papier zu dringen vermögen, erzeugten keine Fluoreszenz des Pergamentes ${ }^{2}$ ).

Versuche einer Fluoreszenzerregung durch sekundäre Strahlen, die von metallischen Fenstern an Röntgenröhren ausgehen, wurden nicht gemacht.

\section{Versuche mit Teslastrahlen.}

Wenn zwischen zwei Polen ein elektrischer Strom von sehr hoher Spannung und großer Frequenz übergeht, so entstehen Schwingungen im Äther, die eine Vakuumröhre ohne

1) Die Lindemannsche Röhre. Fortschritt auf dem Gebiet der Röntgenstrahlen, Bd. XVII, Heft 4, 1911, S. 225. Von Prof. Albers Schönberg. - Die Röhre ist erhältlich bei der Firma C. H. F. Müller, Spezialfabrik für Röntgenröhren, Hamburg.

2) Diese Versuche sowie die mit Teslastrahlen und Radium wurden im physikalischen Laboratorium der Universität München ausgeführt. Für die gütige Erlaubnis der Benutzung der Apparate sei Herrn Prof. Dr. L. Graetz und für die gefällige Beihilfe dem Assistenten Dr.-Ing. J. Wiesent bestens gedankt. - Der Firma C. H. F. Müller, Hamburg, die kostenlos eine Lind e mann sche Röhre zur Verfügung stellte, sei gebührender Dank ausgesprochen. 
elektrische Zuleitungselektroden im Innern zum Leuehten bringen ${ }^{1}$ ).

Es wurden nun Streifen einer alten, radierten Pergamenthandschrift in eine evakuierte Röhre eingeschmolzen; verschiedene Male wurde eine Röhre mit solchen Streifen allmählich luftleer gemacht. Dies Pergament erschien in bläulichem Licht. Es konnte aber nicht festgestellt werden, ob das Pergament fluoreszierte, da die übrigen sich bildenden sichtbaren Strahlen im Innenraum der Röhre eine etwa vorhandene Fluoreszenz überdeckten. Bemerkenswert ist, daß das Pergament unter der Wirkung des Teslalichtes keine Veränderungen erkennen ließen.

\section{Versuche mit Kathodenstrahlen ${ }^{2}$ ).}

Bereits an anderer Stelle $^{3}$ ) wurde auf die etwaigen Verwendungsmöglichkeiten der Len ard-Strahlen, also der Kathodenstrahlen, hingewiesen. Ihr vielseitiges Fluoreszenzerregungsvermögen und ihre Durchschlagskraft ließen nämlich erwarten, daß diese Strahlen auch solche Teile eines Palimpsestes zur Fluoreszenz erregen würden, die durch Ultraviolett nicht angeregt werden, so z. B. die braunen Flecken der Gallussäuretinktur ${ }^{4}$ ). Wenn durch eine sogenannte Lenardröhre bei hohem Vakuum hochgespannter Gleichstrom geschickt wird, so entstehen an der Kathode Strahlen, die sich senkrecht zur Austrittsfläche geradlinig fortpflanzen. Der Ort der Anode ist von keinem Einfluß. Die Kathodenstrahlen können nun durch ein kleines Fensterchen, das mit einer dünnen Aluminiumfolie

I) Etienne Fodor, Teslaversuche, Hartleben - Wien.

2) Die Kathodenstrahlen. Von G. C. Schmidt, 1907. Die Kathodenstrahlen. Von P. Lenard, Nobelvorlesung I9o6.

3) Die Photographie historischer Dokumente, 1. c., S. 80.

4) Der Verfasser konnte zunächst in Ermanglung geeigneter Apparate die Versuche nicht ausfuhren. Dies wurde erst durch das gütige Entgegenkommen von Herrn Dr. C. T. Fischer, Professor an der Technischen Hochschule in München, möglich. Ihm sei denn an dieser Stelle ein aufrichtiges Dankeswort ausgesprochen. 
abgeschlossen ist, ins Freie geleitet werden. Bei ihrem Auftreffen auf das Pergament erregen sie dieses zu rosaroter Fluoreszenz. Diese Erscheinung kann aber photographisch nicht festgehalten werden, da die Aluminiumfolie stets schneller durchschmilzt, als eine photographische Aufnahme der immerhin schwachen Fluoreszenz möglich ist ${ }^{1}$ ). Dies gelingt jedoch beim Einführen des Pergamentes in eine Kathodenröhre.

Die Fluoreszenz ist hier die gleiche wie bei den Lenardstrablen und kann mit einer orthochromatischen Platte und einem Objektiv von F: 4,5 in einer Minute aufgenommen werden.

Die Kathodenstrahlen erzeugen bei ibrem Auftreffen auf das Pergament eine Erwärmung, die zu einer Bräunung desselben führen kann. Dieser Übelstand konnte durch eïne intermittierende, periodische Bestrablung behoben werden, indem in den Stromkreis eine Funkenstrecke eingeschaltet wurde.

Die Versuche fanden zunächst mit kleinen Streifen eines künstlichen Palimpsestes statt, das aber nach mittelalterlichem Verfahren hergestellt wurde. Weitere Versuche mit einem großen Vakuumrezipienten mit planparalleler Deckplatte aus Glas führten zu keinem anderen Ergebnis. Das Instrumentarium ist sehr teuer. .

\section{Versuche mit Anoden- bzw. Kanalstrahlen.}

Wenn eine Kathode so in ein Entladungsrohr eingebaut ist, $d a ß$ dieses dadurch in zwei Teile geteilt ist und die Kathode nach Art eines Siebes durchlöchert ist, so treten aus diesen Öffnungen nach rückwärts positive Strahlen aus, die Kanalstrahlen genannt werden.

Unter diesen Strahlen leuchtet gewöhnliches Pergament schwach gelb. Flecken von Gallustinktur beginnen erst dann zu leuchten, wenn bereits Kathodenstrahlen entstehen.

Versuche mit Salzanoden- und Striktionsstrahlen wurden nicht gemacht.

I) Vielleicht wäre eine Folie aus Beryllium fester als aus Aluminium. 


\section{$-52-$ \\ Versuche mit Radium.}

Es wurde im Dunkeln geprüft, ob ein starkes Präparat von 0,723 Radiumbariumbromid Pergament zur Fluoreszenz erregen würde. Der Versuch war negativ.

Es sei noch erwähnt, daß bei all den genannten Versuchen eine Prüfung auf ultraviolette Fluoreszenz nicht vorgenommen wurde, da anzunehmen ist, daß ibr Vorhandensein keinen praktischen Vorteil bieten würde.

Nun ein Blick in die Zukunft. Worin würde ein neues, besonders wertrolles Hilfsmittel der Palimpsestfluoreszenzphotographie bestehen? In einer einfachen und billigen Lichtquelle, die ultraviolette Strahlen unter $200 \mu \mu$ in großen Mengen liefert. Diese Strahlen würden auch von solchen äußerst geringen Tintenresten absorbiert werden, die von Strahlen größerer Wellenlänge durchdrungen werden und daher keinen oder nur mangelhaften Dunkeleffekt liefern.

Von Nutzen könnte vielleicht die Verbindung der Flúoreszenzphotographie mit der Hale-Deslanderschen spektroheliographischen Methode zur Unterscheidung des fluoreszierenden Pergamentes von einer mit Schwefelammonium behandelten und auch fluoreszierender Schrift sein. Der erforderliche Zeitund Kostenaufwand wäre groß.

\section{Literatur:}

Über Lumineszenz durch Kathodenstrahlen. Von W. Wiedemann und G. C. Schmidt. Wiedem. Ann., Bd. 56, S. 18-25, 1895 .

Über Lumineszenz. Von E. Wiedemann und G. C. Schmidt. Ann. d. Phys., Bd. 56, S. 604, 1895 .

Über Lichtemission organischer Substanzen im gasförmigen, flüssigen und festen Zustand. Von E. Wied emann und G.C.Schmidt, Ann. d. Phys., Bd. 56, S. 18, 1895 .

Öber Lumineszenz. Von W. Arnold. Wiedem. Ann., Bd. 6I, S. $31_{3} 3,1897$; E. W i e d e m a n n, Festschrift ${ }_{1}$ I2, S. ${ }_{36}$, Erlangen, Igor. 


\section{Die Verfahren zum Abdecken der Sekundärschrift der Codices rescripti.}

0 . Gradenwitz und E. Pringsheim ${ }^{1}$ ) haben, wie früher erwähnt, zuerst die Abdeckung der Neuschrift der. Codices rescripti durchgeführt. Nach ihnen wird mittels einer Eosinplatte mit Überexposition und flauer Entwicklung das Negativ $A$ hergestellt. Auf diesem Negativ $A$ treten die stärkeren Reste der sichtbaren Erstschrift zurück, so daß das Planum eine einheitliche Deckung erhält. Es folgt dann eine zweite Aufnahme des Originals mit einer gewöhnlichen Bromsilberplatte (Negativ B), auf der auch die schwächeren, sichtbaren Spuren der Erstschrift deutlich hervortreten. Von dem Negativ $B$ wird ein Diapositiv $B I$ hergestellt. Dieses wird mit dem Negativ $A$ so vereinigt, daß die dunkle Neuschrift die entsprechenden hellen Stellen im Negativ $A$ zudecken. Die vereinigten Platten werden mit Durchsichtbeleuchtung neu aufgenommen. Es entsteht das Negativ $C$. Dieses liefert die endgültige Kopie, auf der die Erstschrift dunkel, die Neuschrift hell erscheint.

O. Mente und A. Warschauer ${ }^{2}$ ) haben diesem Verfahren eine andere, neue Form gegeben. Mit einer panchromatischen Platte und Orangefilter wird das Negativ $A$ hergestellt, mit einer photomechanischen Platte und Blaufilter Negativ $B$. Von $A$ wird ein Diapositiv hergestellt und mit dem Negativ zur Deckung gebracht. Beide dienen zur Herstellung eines vergrößerten Bildes auf Bromsilberpapier. Man kann aber auch das Negativ $A$ auf eine Sannsche Folie oder auf gewöhnliches Zelloidinpapier kopieren, dann die Kopie abnehmen und mit einem Diapositiv von Aufnahme $B$ zur Deckung bringen und weiter kopieren. Man erhält dann natürlich das Bild der alten

I) Verhandl. d. Phys. Gesellsch. Berlin, Bd. 8, S. 58, 1894.

2) Die Photographie in der archiv. Praxis. Von O. Mente und A. Warschauer. Mitteilungen der Königl. Preuß. Archivverwaltung, Heft 15, 1909. - Die Anwendung der Photographie für archivale Praxis. Von O. Mente und A. Warschauer. Verlag S. Hirzel, Leipzig roog. 
Schrift weiß auf schwarzem Hintergrund, also negativ. Diese negative Kopie kann direkt gelesen werden, sie kann aber auch zum Kopieren eines Positives dienen.

Mit den älteren Verfahren gelingt es aber erfahrungsgemäß nur schwer, beide Schriften in entgegengesetzten Helligkeitswerten gleichmäßig wiederzugeben. Dies wird leichter auf folgende Weise erzielt ${ }^{1}$ ). Das Original wird mit einer tagesähnlichen Lichtquelle (Wotan-Verico-Lampe, SiemensSchuckert) nicht sehr stark beleuchtet und mit einer orthochromatischen Zeitplatte aufgenommen. Von diesem Negativ $A$ wird eine Kopie auf einem Diapositivfilm (Entwicklungsfilm²)) hergestellt. Dieses Diapositiv wird mit Quecksilberchlorid gebleicht und mit einer gewöhnlichen Kopie auf Papier von einem Negativ $(B)$, das mit dem Fluoreszenzverfahren gewonnen wurde, zur konkruenten Deckung gebracht. Die vereinigten Positive werden mit auffallender, kräftigęr Beleuchtung neu aufgenommen. Es entsteht Negativ $C$. Von diesem wird die endgültige Kopie gewonnen. Diese Kopie kann mit dem Diapositiv $A I$ nochmals abgedeckt werden, wodurch die Erstschrift besonders stark herrortritt (doppelte Abdeckung, Abb. 21).

\section{Die unsichtbare Anfärbung ${ }^{3}$ ).}

Alle bisher besprochenen Objekte sind selbstdifferenzierend. Ihre photographische Darstellung setzt chemische Unterschiede voraus, auf der die Fluoreszenz des einen Teils und die Nichtfluoreszenz des anderen beruht. Es gibt aber selbstverständlich Objekte, denen im gesamten keine Fluoreszenz zukommt oder deren einzelne Teile gleichartig fluoreszieren. Es sind in beiden Fällen nicht selbstdifferenzierende Objekte. Durch eine

I) D.R.P. ${ }^{28} 5^{1} 54$ (Inhaber P. R. Kögel).

2) Chlorbromsilber-Diapositivfilm der Neuen Photogr. Gesellsch., Berlin -Steglitz.

3) Die unsichtbare Anfärbung. Ein neues Hilfsmittel der forensischen Untersuchung. Von Raph. Kögel. Sitzungsbericht der Heidelberger Akademie, Phil.- hist. Klasse, I9I8. 
Anfärbung können sie selbstdifferenzierend werden, sei es, daß ein Teil des Objektes' zur Fluoreszenz befähigt wird, oder daß in einem gleichartigen fluoreszierenden Objekte einem Teile diese Fähigkeit genommen wird. Die Anfärbung kann ehemisch oder auch physikalisch erfolgen, z. B. durch kolloidale Niederschläge.

Unsichtbar kann die Anfärbung genannt werden, weil das

- Objekt nach einer Behandlung mit farblosen Mitteln dem bloßen Auge noch keine Unterschiede der einzelnen Bestandteile des Objektes erkennen läßt. Diese werden erst mittels der Fluoreszenzphotographie sichtbar. Aber auch dann, wenn das Gesamtaussehen des Originals durch die Anfärbung sichtbar verändert wurde, kann sie als solche in ihren spezifischen Wirkungen als Unterscheidungsmittel unerkennbar und in diesem Sinne unsichtbar sein. Man kann im ersten Falle von einer absoluten unsichtbaren Anfärbung, im letzteren Falle von einer relativen unsichtbaren Anfärbung sprechen.

Da es immer leichter ist, die Fluoreszenz eines Objektes zu dämpfen, als einem nicht fluoreszierenden Körper eine solche zu verleihen, so werden die Methoden der Fluoreszenzverdunkelung zuerst zur Ausbildung gelangen.

Der Verfasser suchte nun zunächst einen Beweis der praktischen Durchführung der soeben dargelegten theoretischen Grundsätze zu erbringen.

Ein ziemlich gut fluoreszierendes Blatt Papier war mit einer Speichelschrift beschrieben. Mittels Fluoreszenzphotographie allein konnte sie nicht sichtbar gemacht werden, sei es, weil die sehr dünne Schrift die ultravioletten Strahlen ohne erhebliche Schwăchung durchließ, so daß die darunterliegenden Papierfasern wie das Planum des Papieres leuchteten, oder sei es, daß die Schrift selbst aus irgendeinem Grund gleich dem Planum leuchtete. Das Schriftstück wurde nun auf folgende Weise angefärbt.

Das Blatt wurde während 2 Minuten auf ein $0,25 \mathrm{~g}$ Ferrosulfat in $100 \mathrm{ccm}$ Wasser gelegt und dann zur Abschleuderung 
der überschüssigen Ferrosulfatlösung horizontal zentrifugiert. Auf einzelne Fasern des Papiers schied sich Hydroxyd ab, ebenso auf der Schrift. Dadurch waren aber, besonders bei den Fasern, noch keine Unterschiede im Objekt zu erkennen. Bei einer fluoreszenzphotographischen Aufnahme traten aber gewisse Fasern und die Schrift infolge Fluoreszenzdunkelheit deutlich hervor, wie eine Abbildung am Schluß dieser Schrift zeigt.

\section{Erläuterungen der Beispiele der Palimpsest- und forensischen Fluoreszenzphotographie ${ }^{1}$.}

Abb. 18 u. 19. Die Erklärungen finden sich unter den Abbildungen.

Abb. 20. Aufnahme mit photomechanischer Platte, Kontrastfilter, Quecksilberdampflicht (altes Verfahren).

.Abb. 21. Fluoreszenzaufnahme mit spektralem Ultraviolett von $313 \mu \mu$ und zweifacher Abdeckung. Die dunkle Schrift ist die Erstschrift, die hellere die Zweitschrift.

Abb. 22. Erklärung unter der Abbildung.

Abb. 23. Der bisher unlesbare Text lautet: Iste liber est dominorum de Schüren. Et est una repositura loco[proprio] decretali [bus] [romanis].

Abb: 24. Die Tinte wurde durch Wasser od. dgl. verschleppt. Gewöhnliche Photographie.

Abb. 25. Fluoreszenzaufnahme mit spektralem Ultraviolett von $313 \mu \mu$.

Abb. 26 u. 27. Das vorliegende Beispiel, wie das mit den $A b b .28-31$, soll dartun, daß die Fluoreszenzphotographie in dem auf Seite 47 ausgesprochenen Sinne quantitativ und qualitativ Neues bringt. In Abb. 26 zeigt der Pfeil $a$ Brucb-

I) Die Beispiele der Palimpsestphotographie wurden aus Codices der Bayerischen Staatsbibliothek München gewählt, da diese Handschriften allgemein zugänglich sind und daher ein Vergleich der Aufnahmen mit den Originalen leicht möglich ist. 
stücke einer Zahl, aufgenommen nach der älteren, dem Fall entsprechenden Methode. Die Auslegung dieser Zahl für 2 kann vom Richter, Staatsanwalt oder Verteidiger wegen ungenügender objektiver Sicherheit zurückgewiesen werden. Abb. 27 zeigt das sichere Vorhandensein der Zahl 2.

Das Original wurde mit prismatischem Ultraviolett von $313 \mu \mu$ beleuchtet und bei einer 24 stündigen Exposition mit einem Mikroobjektiv F : 4 aufgenommen. Eine naturgroße Aufnahme des Originals mit dreistündiger Exposition, gleicher Beleuchtung und einem gewöhnlichen Objektiv von F : 4,5 (TessarZeiß) als Vorversuch hatte bereits erkennen lassen, daß ein für die Fluoreszenzvergrößerung erfolgreicher Fall vorliegen würde.

Abb. 28 zeigt einen Teil einer radierten Quittung, aufgenommen mit den früher gebräuchlichen technischen Hilfsmitteln. In Feld 1, 2, 3 u. 4 befinden sich radierte Zahlen. Feld 3 u. 4 enthalten die Gesamtsumme. Abb. 29 zeigt nun durch Fluoreszenzaufnahme mit $313 \mu \mu$ diese Schlußsumme, die auf 13 lautet. Etwas weniger deutlich kamen die Zahlen 6 in Feld 1 und 50 in Feld 2. In Abb. 30 ist die Zahl 6 besser erkennbar, in Abb. 31 dagegen die Null. Am oberen Teil der Zahl 5 war das Papier auch durchlöchert. Immerhin bestätigen auch die vorhandenen Reste das Vorhandensein der Zahl 5, die bereits durch die Schlußsumme gefordert war. Die Zahlen der Quittung, die nämlich oberhalb der Felder 1 und 2 stehen, geben ihrerseits auch die Summe 6,50. Die wirkliche Gesamtsumme 13 erfordert nun als Ergänzung den Betrag von 6,50. Damit ist die Zahl 5 bereits gegeben.

Abb. $32-35$ zeigen Ausschnitte aus verschiedenen Schriftstücken mit weißem Papier. Abb. 32 gibt eine gewöhnliche Aufnahme mit sichtbaren Strahlen. Die Papierstreifen von 2 und 3 waren nicht streng weiß. Der Unterschied war gerade merkbar, hätte aber doch von manchem in Abrede gestellt werden können.

Abb. 33 zeigt eine direkte Ultraviolettaufnahme mit Strahlen von $313 \mu \mu$. Das Original wurde unmittelbar mit einer Quarz- 
lampe beleuchtet und ein Silberfilter vor das Quarzobjektiv gebracht. Abb. 35 zeigt eine Ultraviolettaufnahme mit $253 \mu \mu$.

Abb. 34 zeigt eine Fluoreszenzaufnahme der gleichen Papierstreifen mittels prismatisch zerlegtem Cltraviolett von $313 \mu \mu$. Ein Vergleich der Abb. 34 u. 35 läßt den Unterschied aller Papierstreifen deutlich erkennen, bis auf die der Nummern 2 u. 5. Die beiden Papiere waren in der Tat auch sehr gleichartig in ihrer Zusammensetzung. Eine direkte Ultraviolettmikroaufnahme (hier nicht wiedergegeben) ließ jedoch eine verschiedene Lagerung der Fasern erkennen. Der Unterschied ist analog dem in den Abb. 37 u. 38, die aber Fluoreszenzmikroaufnahmen von Papierstreifen sind, die auch durch die Abb. 38 in Naturgröße mittels Fluoreszenz dargestellt werden. Abb. 36 zeigt diese Papierstreifen gleichzeitig auf einer Platte aufgenommen. Ein Vergleich der Abb. 36,37 u. 38 läßt auf den ersten Blick es kaum glaublich erscheinen, daß Abb. 37 u. 38 die Streifen von Abb. 36 wiedergibt. $\mathrm{Daß}$ dem doch so ist, beweist offenkundig ein besonders deutliches Hervortreten der Fasern, auf die mit Pfeilen hingewiesen ist. Besonders in Abb. 38 kann man deutlich einige stark leuchtende Fasern von $36 / 2$ erkennen.

Abb. 39 stellt wiederum Vergleichsaufnahmen von Ausschnitten rerschiedener Papierstreifen dar. I gibt eine gewöhnliche Aufnahme mit sichtbarem Licht, II eine direkte Ultraviolettaufnahme mit $313 \mu \mu$ und III eine mit $253 \mu \mu$. Zu beachten sind zunächst die Schriften von Nr. 3 und 4 . Es fragt sich, ob die beiden Schriften mit gleicher Tinte ausgeführt sind. Nach I scheint 3 dunkler zu sein als 4 . Dies kann aber auch sowohl im Original als auf der Photographie auf optischer Täuschung beruhen. Denn eine dünne Schrift erscheint in ihrem Gesamteindruck leicht heller als eine breitere. In III erscheint die Schrift von 3 heller als die von 4 . Die beiden Tinten sind also verschieden. Der Befund durch III zeigt, daß die umgekehrten Helligkeitswerte in I aber doch auf einer wirklichen Verschiedenheit beruhen. 
Die Nrn. 17 und 18 zeigen in II und III eine gleiche Ultraviolettreflexionsfähigkeit des Papiers, aber eine sehr verschiedene der Tinten. Die von 18 versinkt bereits in dem Planum des Papiers, während die von 17 eine größere Ultraviolettreflexionsfähigkeit zeigt als das Papier selbst.

In II zeigt ein Pfeil zwischen 8 und 9 auf schwache Umrisse einer Zahl 2. Die Zahl liegt unter einer mehrfach aufgetragenen schwarzen und roten Tinte. Es muß bemerkt werden, daß ein solches Ergebnis nur selten erhofft werden darf und nur auf ganz zufällige, außergewöhnliche Verschiedenheiten der Tinten beruhen kann.

Andere Ergebnisse der Abb. 39 sollen weiter nicht besprochen werden, da ein Autotypiedruck die Einzelheiten nicht deutlich genug wiederzugeben vermag.

Abb. 40. Der Poststempel war nur mehr schwach zu erkennen. Durch die Fluoreszenz des Bindemittels der Stempelfarbe, das stark diffundierte, trat diese selbst deutlich. hervor.

Abb. 41. Es war die Frage zu lösen, ob der Brief vor oder nach Aufdruck des Ankuniftstempels mit einem besonderen Klebemittel verschlossen worden war. Die hellfeuchtenden Flecken des Klebemittels treten stark hervor. Der Ankunftsstempel liegt über dem Klebemittel. $B B$ sind Linien eines Winkels, der mit dem Winkel des oberen Briefverschlusses kongruent ist. Es .ist der Abdrúck von Resten des nassen Klebemittels' auf einem Löschblatt, das beim Zukleben des Briefes benutzt wurde. Der Buchstabe $N$ liegt auch hier über dem Klebemittel. Also ein zweiter Beweis, daß der Stempel erst nach dem Zukleben aufgedruckt wurde und ein erneutes Zukleben nach dem Abstempeln nicht stattfand. Von juristischer Seite der Fall beurteilt, besagt er, daß ein Zukleben durch den Briefträger, der den Brief erst nach Aufdruck des Ankunftsstempels erhielt, nicht stattgefunden hat.

Abb. 42 zeigt ein Beispiel der "unsichtbaren Anfärbetechnik": der eine allgemeinere, prinzipielle Bedeutung zukommt. Weißes, ziemlich gut fluoreszierendes Briefpapier wurde 
mit Speichel beschrieben. Das Blatt wurde dann während $2 \frac{1}{2}$ Minuten auf eine Lösung von $0,25 \mathrm{~g}$ Eisenvitriol in $100 \mathrm{ccm}$ Wasser so gelegt, daß nur die Schriftseite mit. der Lösung in Berührung kam. Das Blatt wurde dann horizontal zentrifugiert, um die übrige Eisenvitriollösung abzuschleudern. Damit war die Schrift unsichtbar angefärbt, visuell aber doch nicht wahrnehmbar. Daß eine wirkliche Anfärbung vorliegt, und daß das Eisenvitriol nicht etwa nur physikalisch zwischen Papierfasern lag, die durch die Schreibfeder aufgelockert waren, zeigen die Flecken. Es sind Speicheltropfen; die zufällig auf das Papier fielen und keine mechanische Wirkung ausüben können. Auch einzelne Papierfasern kamen dadurch deutlicher zum Vorschein, daß die einen Eisenvitriol adsorbierten, die anderen aber nicht.

Eine Fluoreszenzaufnahme mit Strahlen von $\lambda=366 \mu \mu$ (Quarzlampe, Blauuviolglasküvette, Antrachinonfilter) brachte den Text wieder, bis auf wenige Buchstaben, von denen überhaupt nicht sicher ist, ob sie nicht mit leerer Feder geschrieben wurden. Eine gewöhnliche Aufnahme ist total leer. Es ist nun bekannt, daß Speichelschriften mitunter durch Tintenbildung visuell sichtbar gemacht werden können. Dies beeinträchtigt aber nicht die prinzipielle Bedeutung des vorliegenden Verfahrens.

Je nach dem Objekt wird die unsichtbare Anfärbung mittels chemischer Verbindungen oder durch kolloidale Adsorption (Gerbung u. dgl.) durchzuführen sein. Dabei wird man sowohl fluoreszierende als nicht fluoreszierende Objektträger benutzen. Die Fluoreszenzphotographie im Verein mit der unsichtbaren Anfärbung wird, sowohl in der Technik als in den Naturwissenschaften, besonders in der Medizin, der Forschung neue Bahnen eröffnen. 


\section{Personenverzeichnis.}

Andès, L. 28.

Arnold, W. 52.

Baly, E. 42.

Baumert, G. VIII.

Beetz, W. 9.

Birgitti 48.

Bordier 24 .

Cornu 32.

Gourmont, J. 24.

Cremer, F. 42.

Dennstedt, M. VIII.

Dickson, E. 42.

Eder, J. M. 6, го, I3f., 42.

Ehrle, F. 46.

Engelhardt, E. 47.

Emmerich, G. H. 3 .

Faworski, W. 37 .

Fodor, Etienne 50.

Girard, F. 25.

Goerz, P. C. I4.

Gradenwitz, O. 2, 53 .

Graetz, L. 49.

Grassel, A. VIII.

Grebe, R. 5, 42.

Heller, R. 47.

Henri, V. 24.
Heraeus (Hanau) 23.

Hoh \& Hahne 6.

Hotz-Ostwald 44 .

Kauffmann, H. 42. Kayer, H. I4, 42.

Köhler, A. 5 .

Krumbacher, A. VIII.

Krüß, P. 42.

Küch, R. 2 I.

Lehmann, H. 43.

Lenard I6, 4I.

Ley, H. 4I.

Löhr, L. v. 47.

Luther, R. 42.

Lux, A. 22.

Mente, O. 2, Io, 17.53 .

Meyer \& Stark 42.

Mies, W. 42.

Müller, C. H. F. 49.

Neuhauf, R. I4.

Nogier, Ch. 24 .

Novak, F. 28.

Pauli, E. W. 42.

Perutz, O. 6.

Phole, J. C. I9, 25.

Plotnikow, J. IIf.

Pringsheim, E. 2, 53 .
Ramsauer I6.

Retschinsky, T. 2 I.

Richter, J. Io, I7.

Ritter, J. W. I8.

Schall, Ch. I6.

Schmidt, G. C. 50,52 .

- , H. 42.

Schott \& Cie. I2.

Schuhmann, R. I5.

Siemens-Schuckert II.

Stark 37, 4If.

Tuck, W. 42.

Urban, W. 37.

Valenta, E. Io, I3, 42.

Voigtländer, F. VIII.

Warschauer, A. 2.

Weigert, F. 42.

Westendorp \& Wehner

9.

Wiedemann, E. 52 .

Wiegand, A. 32.

Wiesent, J. 49.

Wolff, O. 47.

Wolfke, W. I9.

Zeiß, C. 13, 16, 28.

Zsigmondy, R. 5 . 


\section{Sachverzeichnis.}

Abdeckungsverfahren 2, 53 . Absorptionsfilter für Ultraviolett $25,33,37$.

Aluminiumfolie $5 \mathbf{x}$.

Anfärbung, unsichtbare 54 .

Anodenstrahlen $5 \mathrm{I}$.

Anthrachinonsulfosaures Natron 33, 40.

Anthrazen 38 .

Aplanat 7 .

Apochromat 7 .

Azolampe 4.

Bariumphosphatkronglas I2.

Baumwollegelb I5. $_{5}$.

Baryt-Leichtflint I3. $_{3}$

Baryt-Kron $\mathbf{I 3}_{3}$.

Beleuchtung, intensive 3 .

Benzol 38.

Beryllium 49.

Blauuviolglas ro, 33 .

Bleiglas I2.

Bogenlicht 3 .

Bor 49.

Brennpunkt, optischer I4.

-, photochemischer I4.

Bromdampffilter $3 \mathbf{r}$.

Bromsilberplatte $5,8, I_{3}, I_{5}$, 36.

-, bindemittelfreie $\mathbf{I}_{5}$.

-, hochempfindliche 36 .

Cellit 46.

Celor 35 .

Chininsulfat 28.

Chlorsilberplatte I3. $_{3}$

Chrysen 33.

Codices rescripti III, 2, 53 .

Cooper-Hewitt-Lampen I2, 2̇.

Diagonale 7 .

Drosselspule 20.

Druckerschwärze 3 .

Edelsteine 47 .

Eisenvitriol 55,60 .

Eosinplatte 53 .

Exposition für Fluoreszenzaufnahmen 36 .

Farbenmischung, subtraktive 5 .

Ferrosulfat 55,60 .

Filter 5, 8 (siehe U.-V.-Absorptionsfilter, Silberfilter).

Filterlampen 34 .

Fixieren 8.

Fluoreszenz der Mineralien 47.

- des Papieres 47.

- - Pergamentes 18, 25.

-, ultraviolette 37 .

- , Wesen der $4 \mathrm{I}$.

Fluoreszeinnatrium 28 .

Folien (Film) 53.

Forensische Praxis $7,10,16,36$, 46.

Gallustinktur 6, 8, 5of.

Gelatine 46. 
Gelbfilter 6, 4I.

Gerbung 46.

Giobertitinktur 37,45 .

Gradation 8.

Hale-Deslandresche Methode $5^{2}$. Halloidsilber 8.

Heraeus-Lampe 24.

Infrarot 9.

Kalzium 48.

Kanalstrahlen $\mathbf{5}$.

Kanariengelb $\mathbf{I}_{5}$.

Kathodenstrahlen 50 .

Klebemittel 59.

Kondensor 19,34 .

Kollektor 5, 19, 26, 34.

Kupfersulfat 33 .

Küvette 6 (siehe Filter).

Lenardstrahlen 50.

Leuchtplatten 28.

Lichtfilter 5 .

Lithium 49.

Lumineszenz I (siehe Fluoreszenz).

Mennige 48.

Metalfalampe 25 .

Metalldrahtlampen 3 .

Mikroobjektive 7,36 .

Miniaturen Io.

Monochromat I4.

Natrium 48.

- , anthrachinonsaures 33,40 .

- , unterschwefligsaures 8 .

Naphthalin $3^{8}$.

Nernstlampe 3 .

Nickellicht II, 33,36 .

Nickelstahl ro.
Nitralampe 4 .

Nitrosodimethylanilin $33,4 \mathrm{I}$.

Öle 47 .

Objektive 7,35 .

- aus Quarz I4.

-, Mikro- 7, 36 .

Ozobromverfahren 37 .

Palimpsest, Definition I.

Papier, Reflexionsvermögen 3 .

Papiere, leuchtende 28.

Papierfaser 46,56 .

Perlen 47.

Petroleum 28.

Phenanthren $3^{8}$.

Phenetol 39.

Phenylendiamin, $\mathrm{p}$ - I6.

Phosphoreszenzschirm 27.

Pinacyanolplatte 9 .

Planare 7 .

Platte $5,7,1_{3}, 1_{5}, 36,53$.

-, bindemittelfreie $\mathbf{I}_{5}$.

-, hochempfindliche 36 .

- , nasse 6 .

-, photomechanische $5,8,15,16$.

Prismen 19, 27.

Quarzlampe I3, I9, 22.

-, von Heraeus 24.

-, Metalfa- 25 .

- W. C. -24.

-, Wechselstrom - 25.

-, Wolfke v. I9.

Radium 5 .

Reagenzien 37, 44 (siehe Gallusund Giobertitinktur, Schwefelammonium).

Rhodanammoniumverbindungen 45 .

Röntgenstrahlen 48 . 


\section{$-64-$}

Salzsäure 44 .

Schwefelammonium 8, 45, 52 .

Schwefelzink 28 .

Selbstinduktanz 4 .

Sensibilisatoren für Ultraviolett I5.

Sidotblende 28.

Silberfilter II, I3, 3I, 36 .

Silikat-Flint 13.

Silizium 48.

Solenoid 20.

Spalt 19, 27.

Speichelschrift 55 .

Spektrum I9, 28.

Ultramarin 48.

Ultrarapidplatte 36 .

Ultraviolettfilterlampe $33 \mathrm{f}$.

--photographie Io.

--projektionsvorrichtung 18.

- papier 16.

--platten, siehe Platten.

Uviolglas, blaues I0, I2, 33 .

-, weißes 5, I3, 3 I.

Uviollampe 12, 22, 34 .

Vakuum, optisches I3. $^{3}$.

Vergrößerung 7 .

Wattverbrauch der Quarzlampe 2 I.

Terpene $4 \mathrm{I}$.

Tiefenreliefs 44 :

Tinten 46, 59.

Toluidindiaminpapier $\mathbf{~} 6$.

Toluol $3^{8}$.

Triphenylmethan 40.

W. C.-Lampe $4,12$.

Wotanlampe 4,54 .

Xylol $3^{8 .}$

Zinnober 48. 

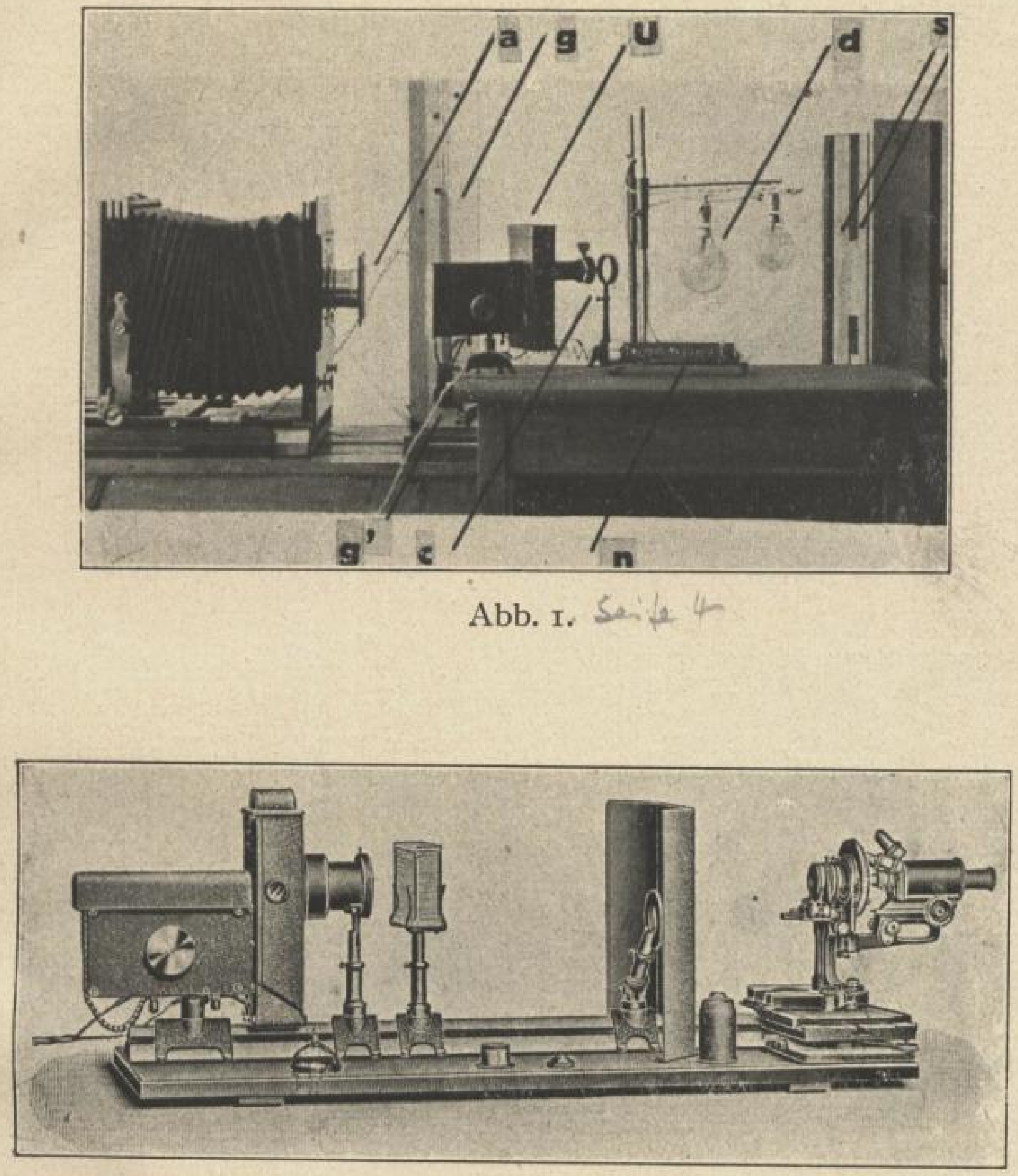

Abb. 2.

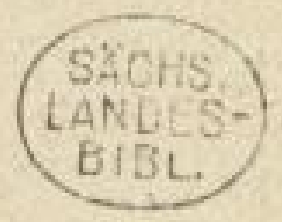

SLUB Wir fohren Wissen. gefördert von der
Deutschen Forschungsgemeinschaft 


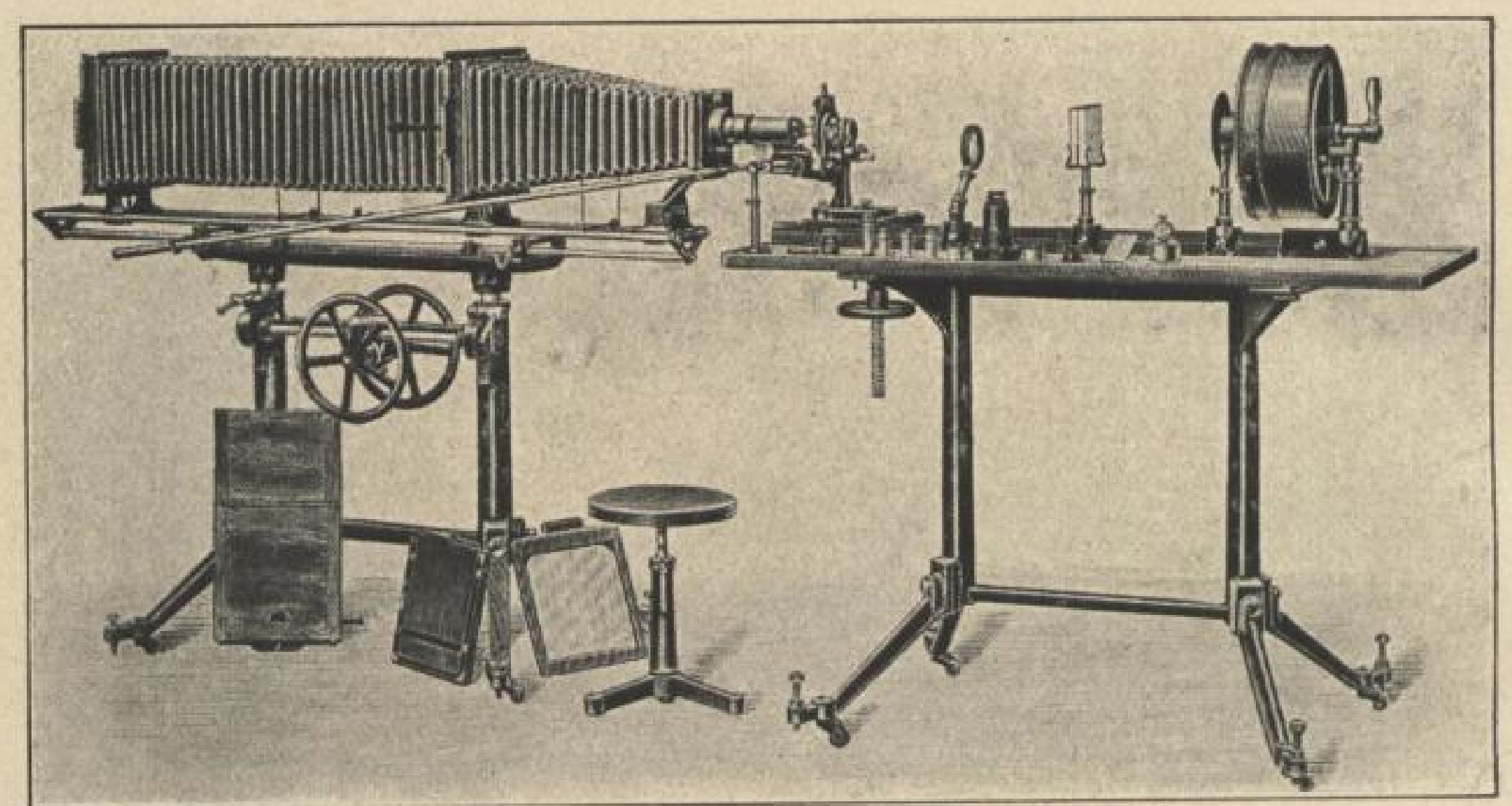

Abb. 3 .

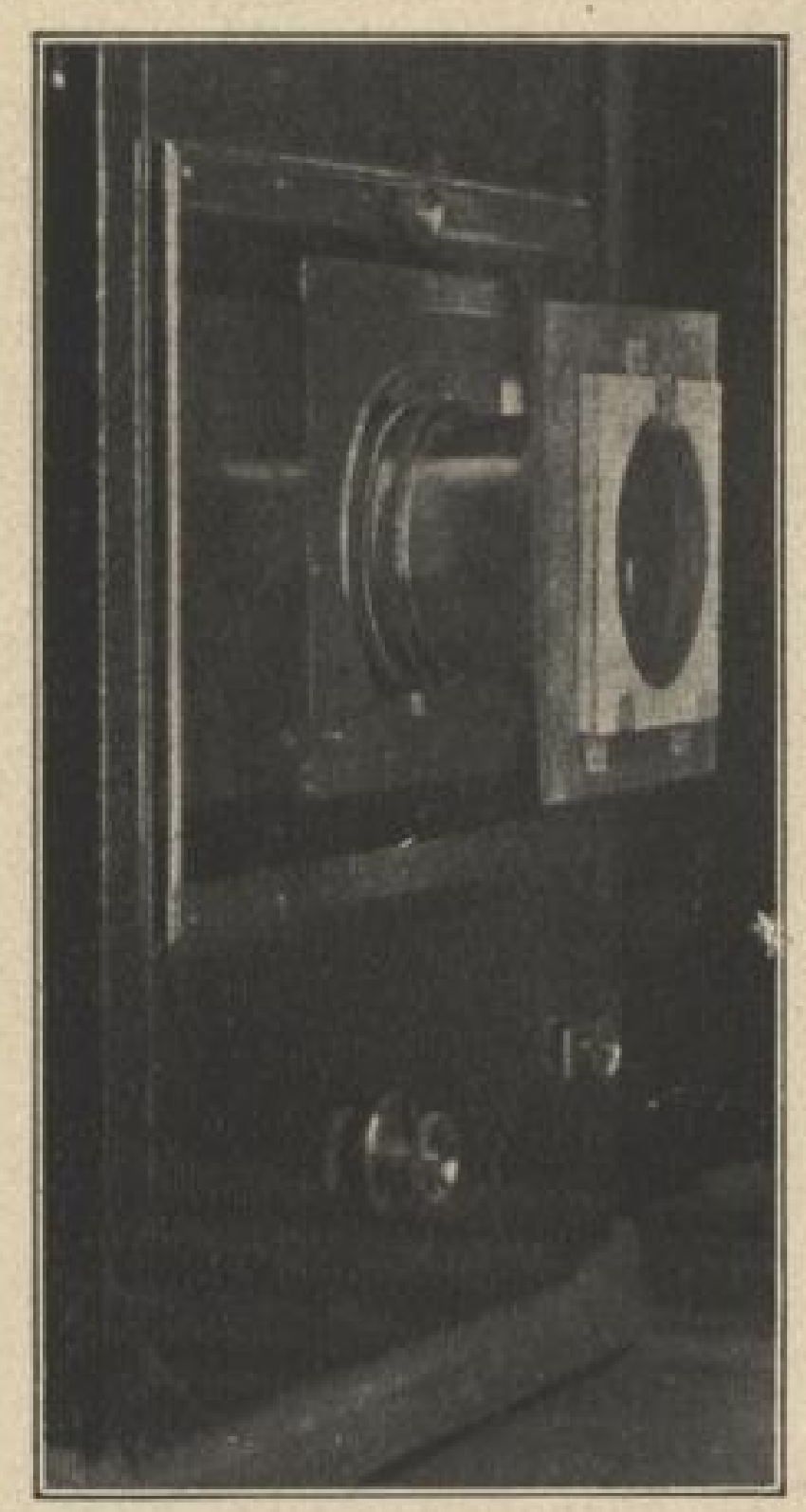

Abb. 4 .

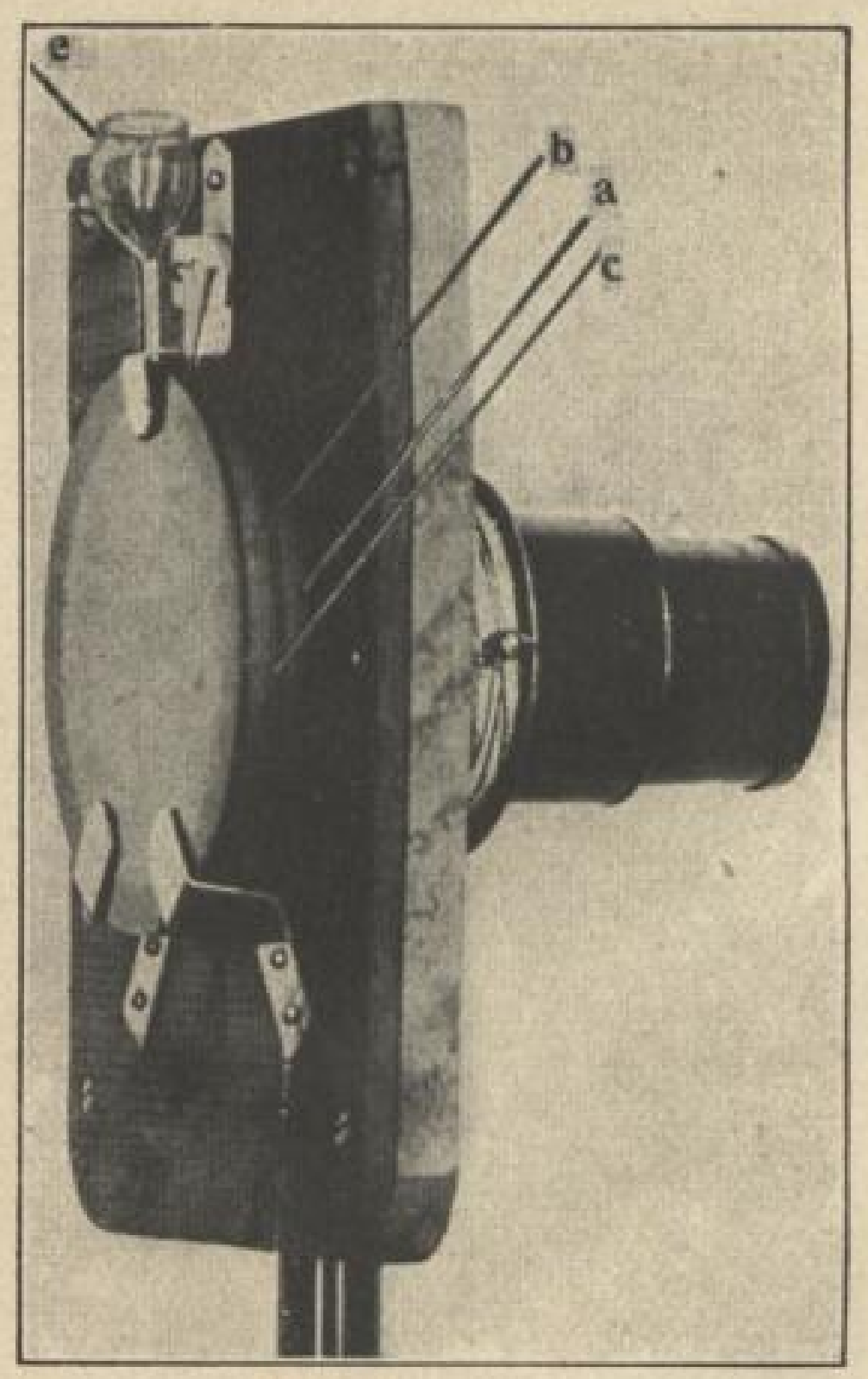

Abb. 5 . 


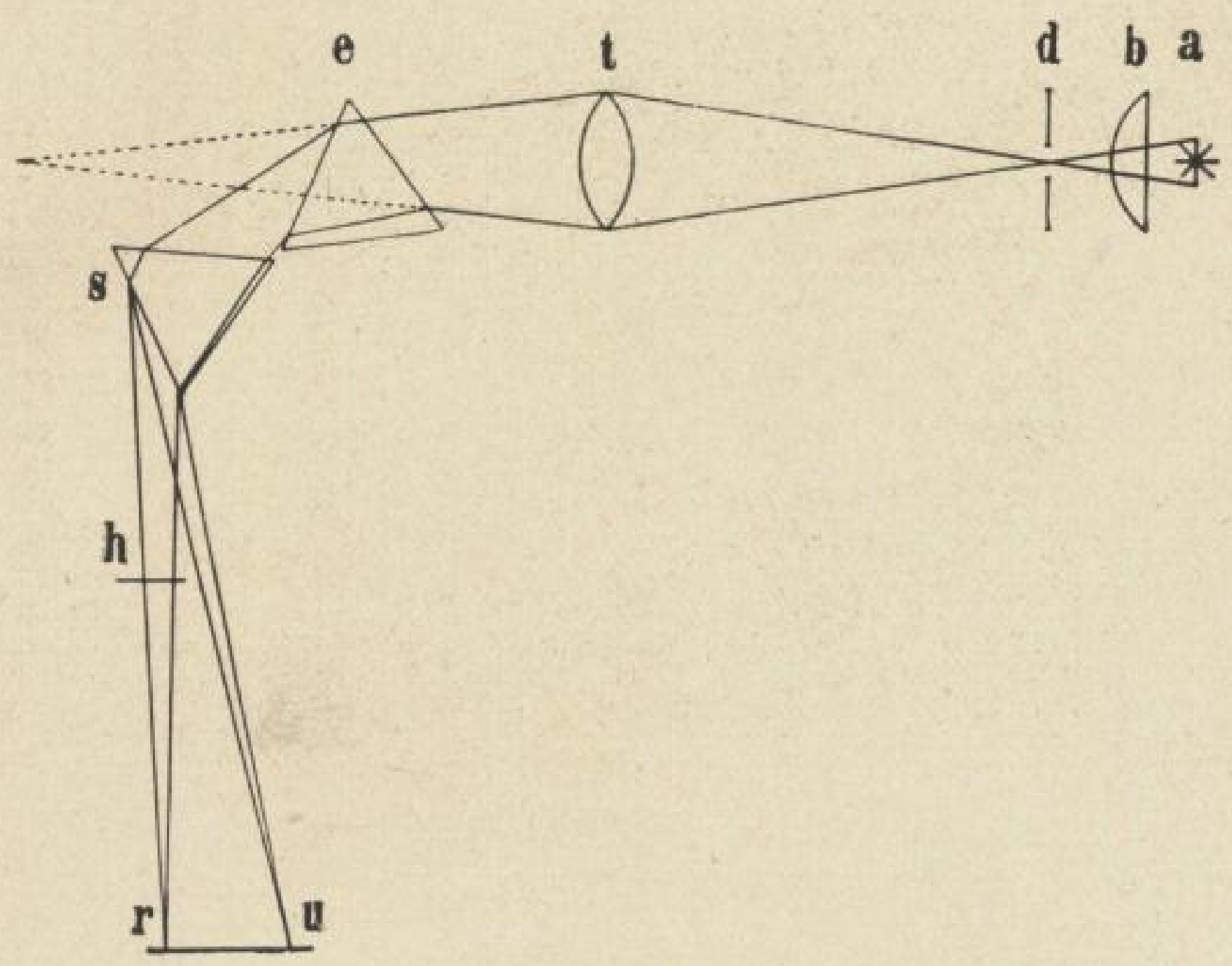

Abb. 6.

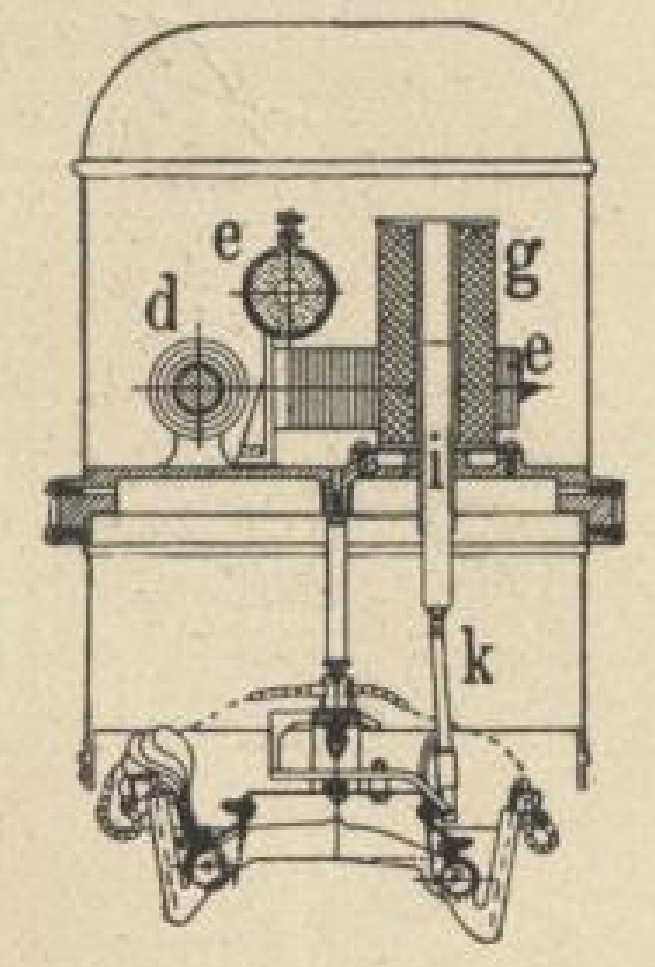

Abb. 7 .

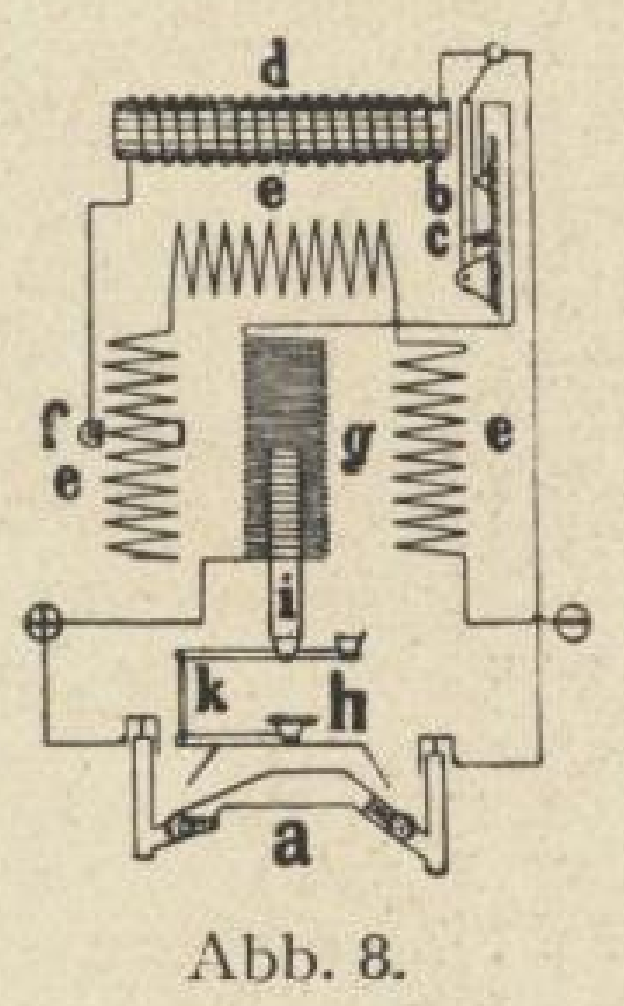




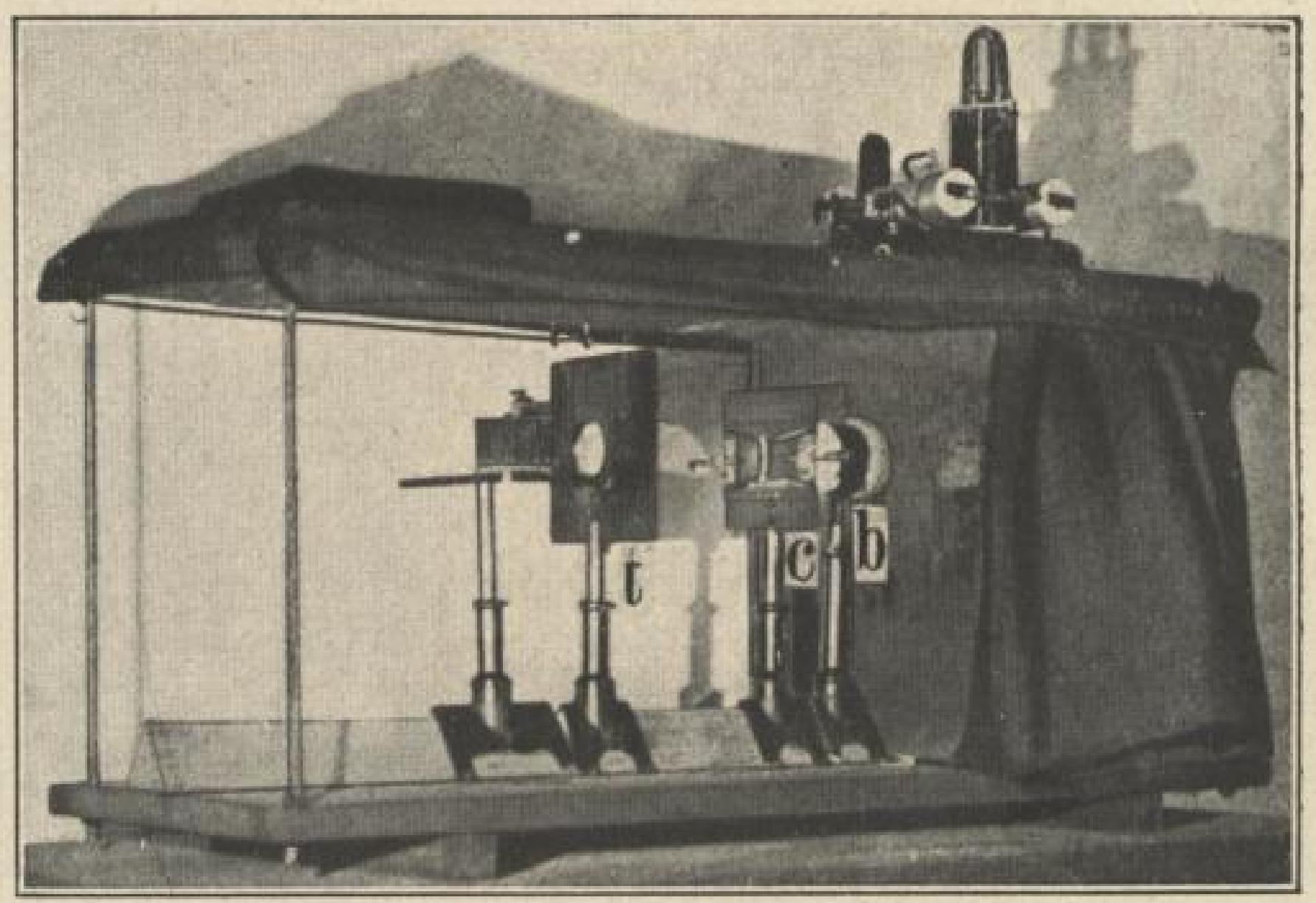

Abb. 9.

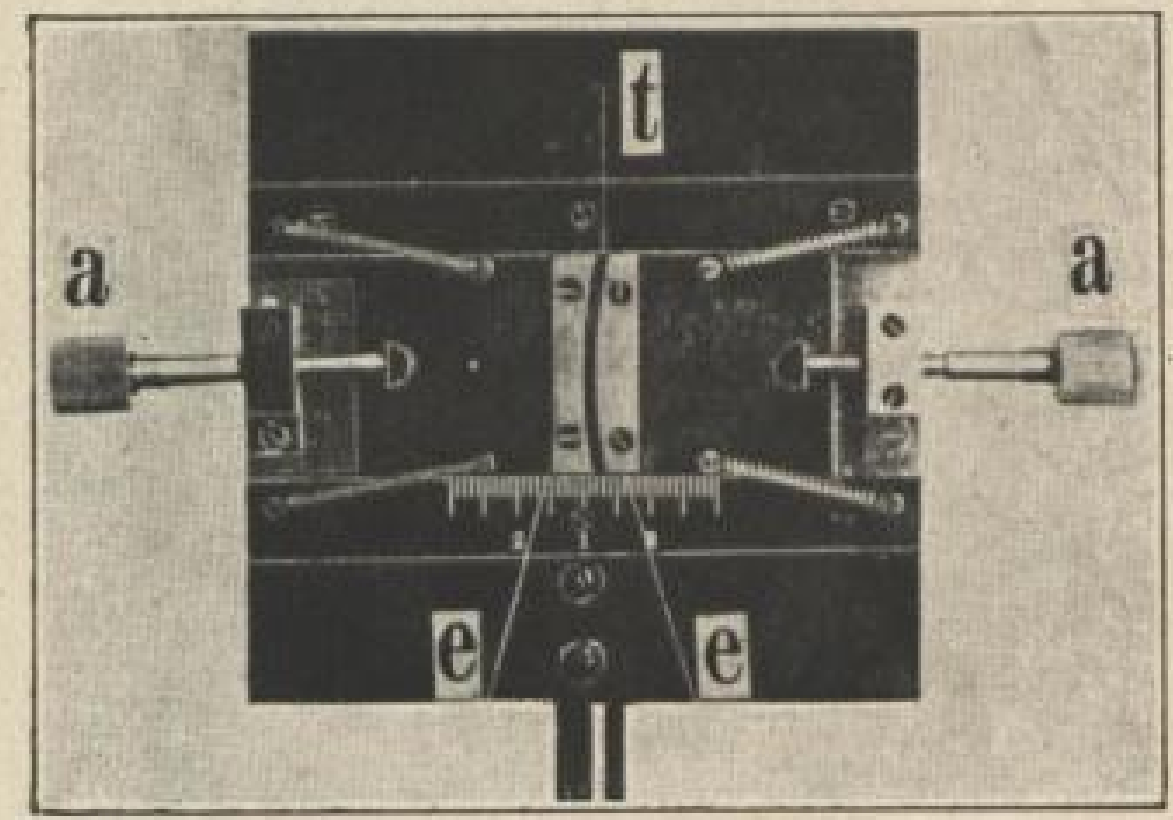

Abb. 1o.

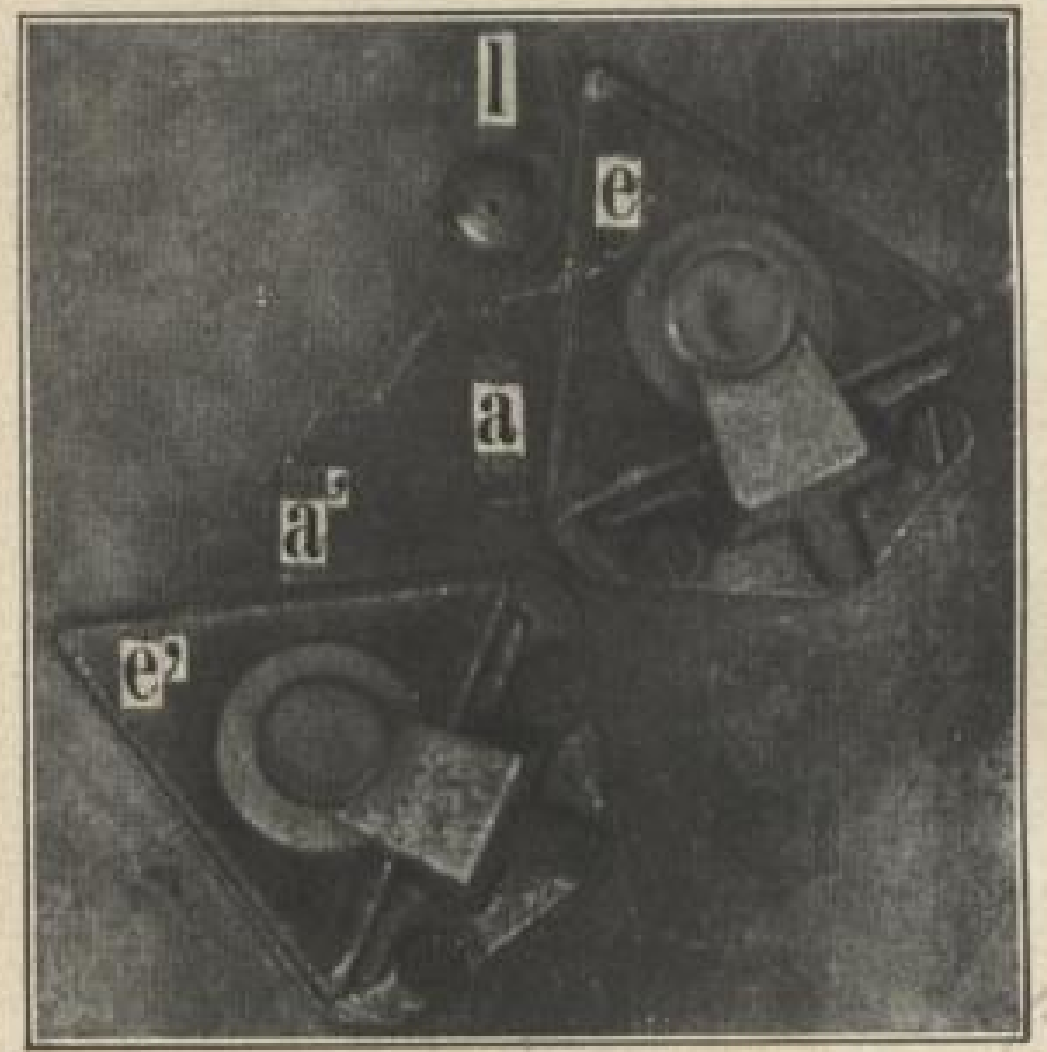

Abb. II.
SLUB

Wir fohren Wissen. gefördert von der $\mathbf{D}=\mathbf{C}$ Deutschen Forschungsgemeinschaft 


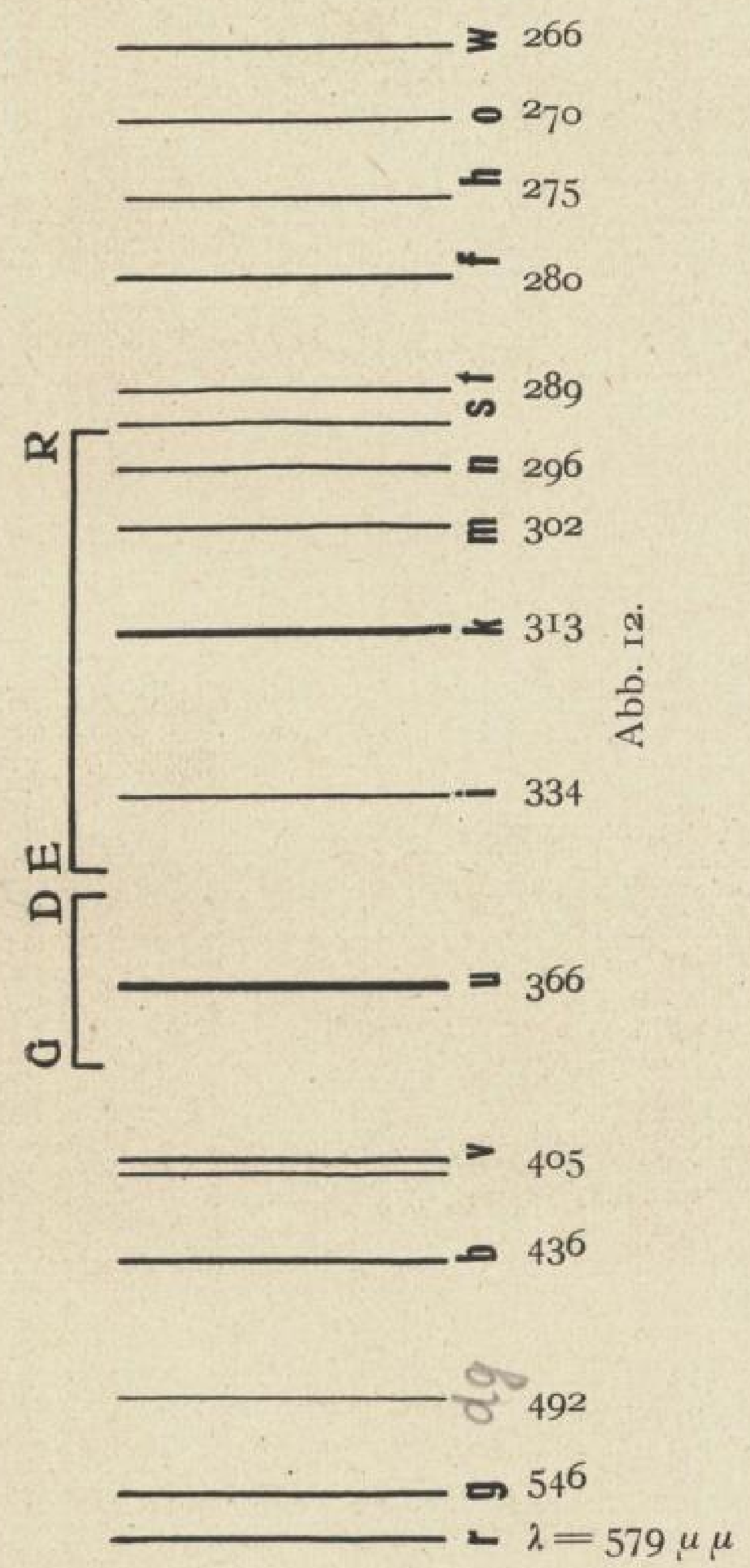




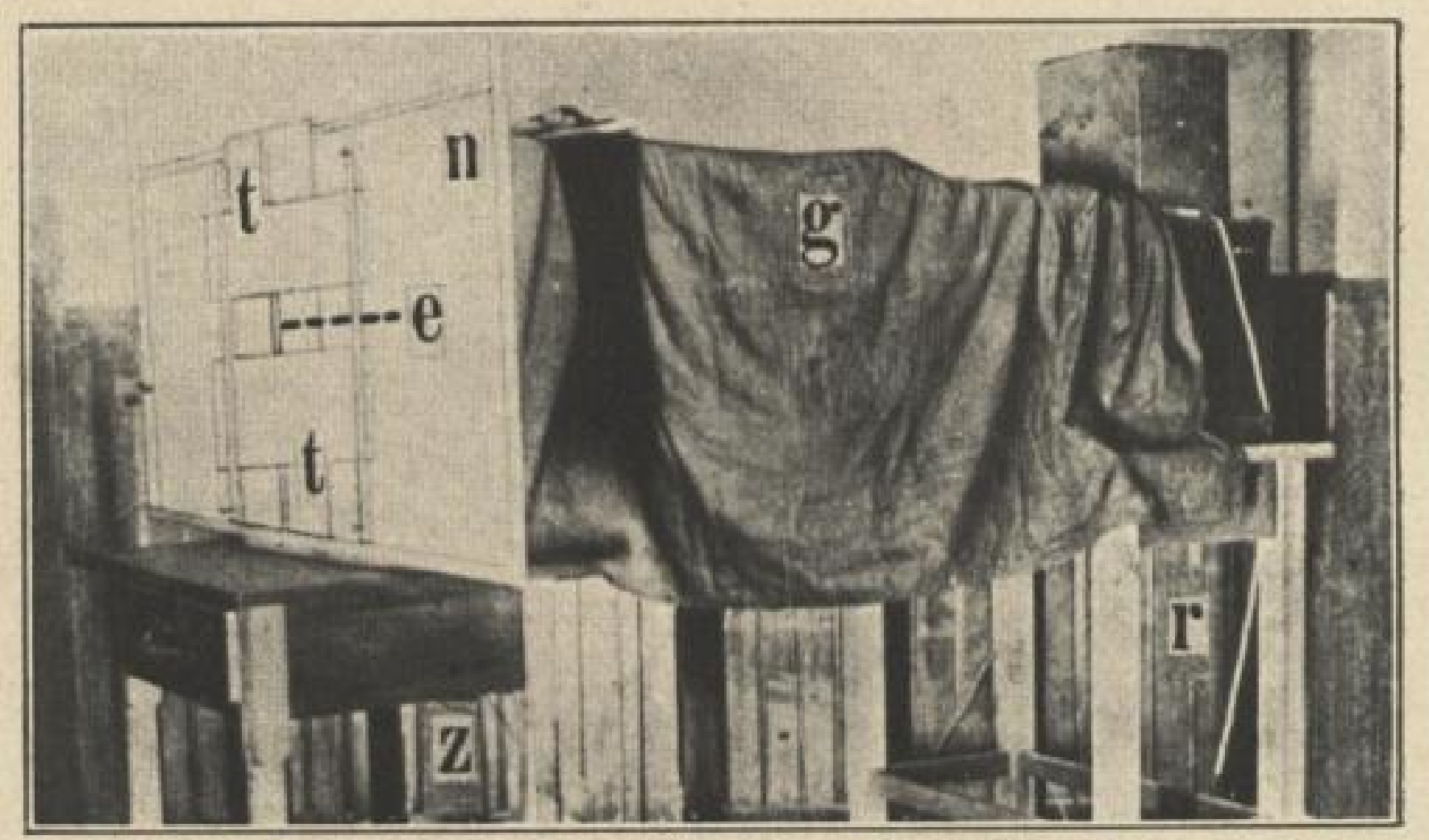

Abb. 13.
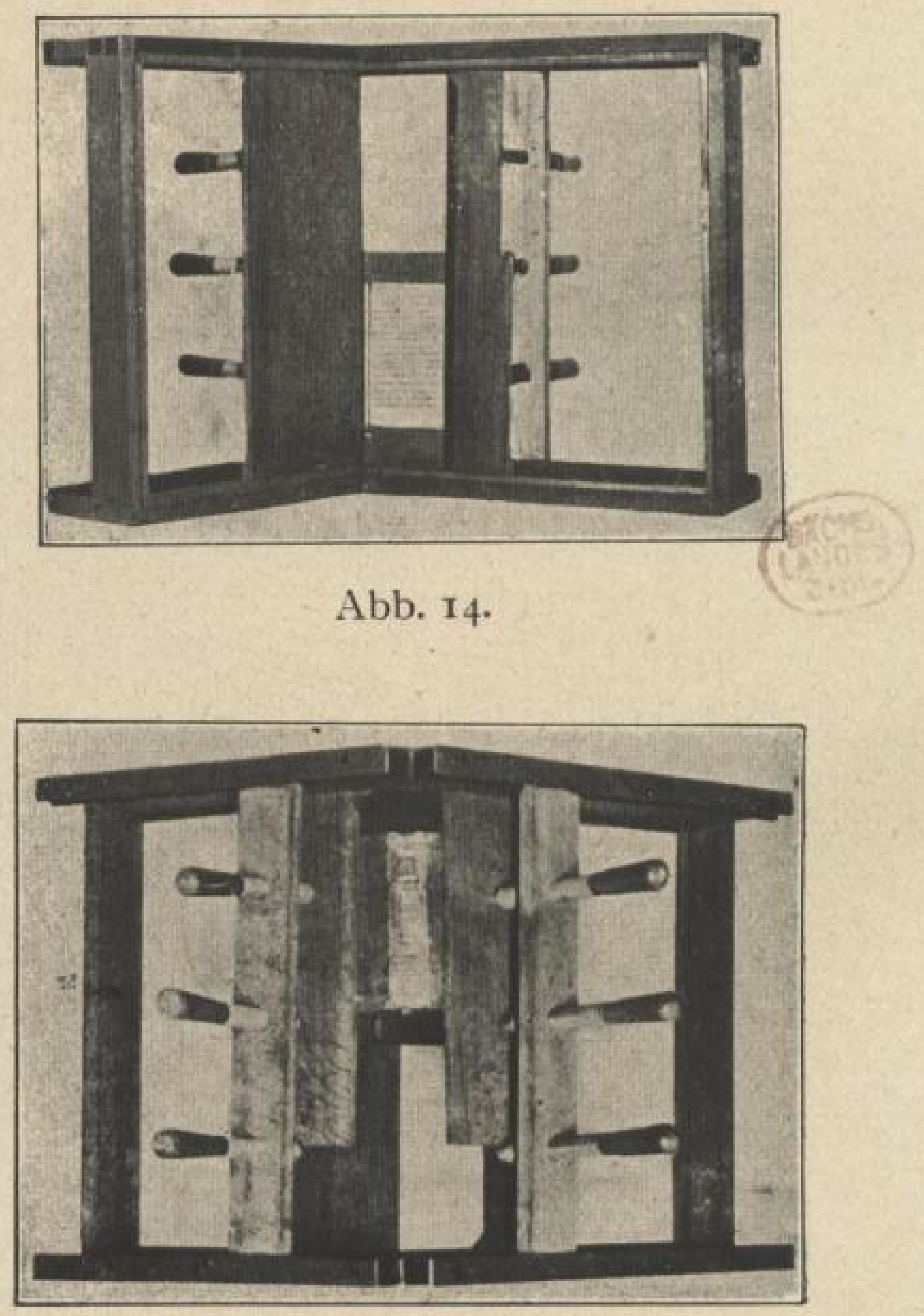

Abb. 15 . 


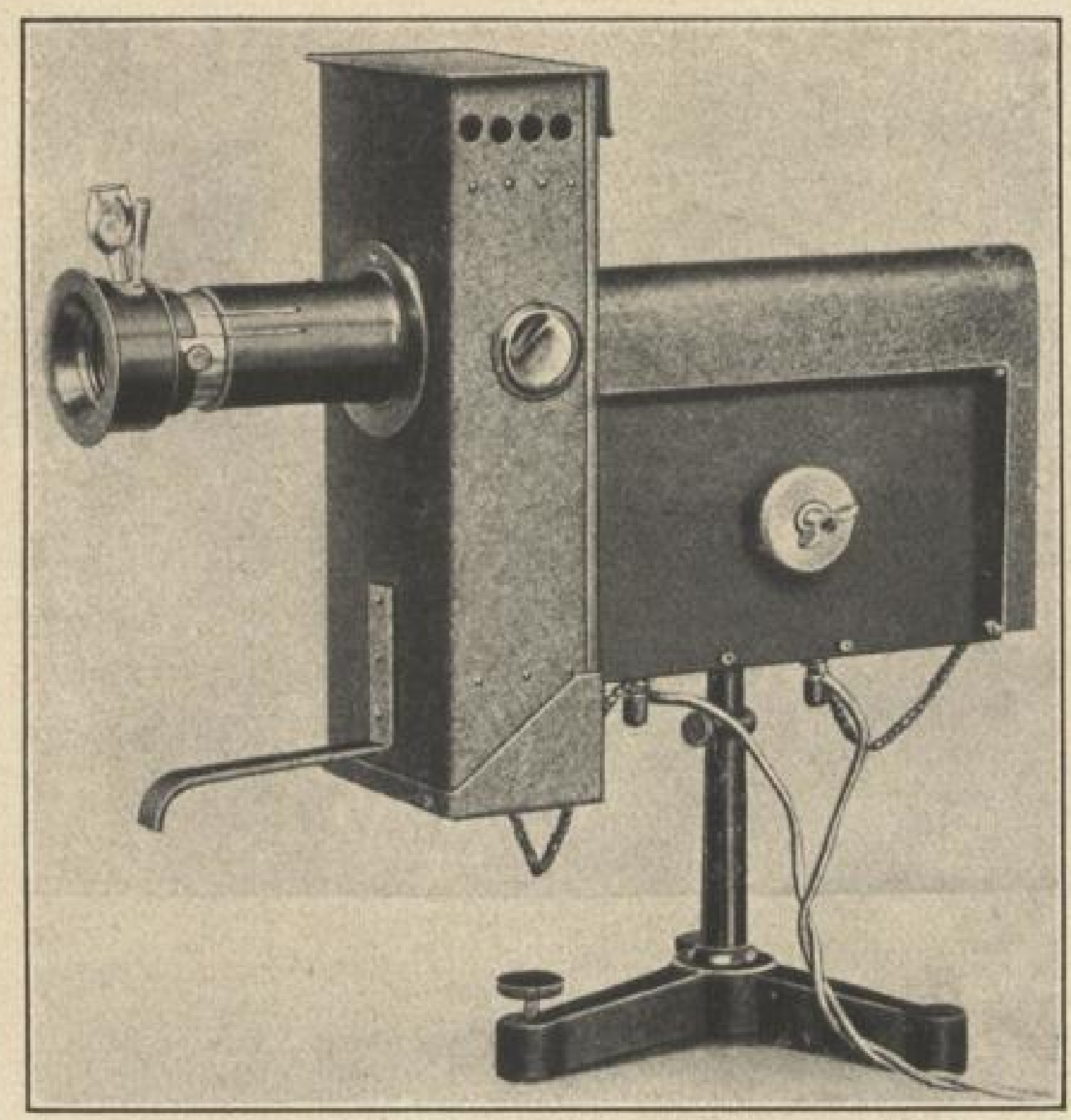

Abb. 16.

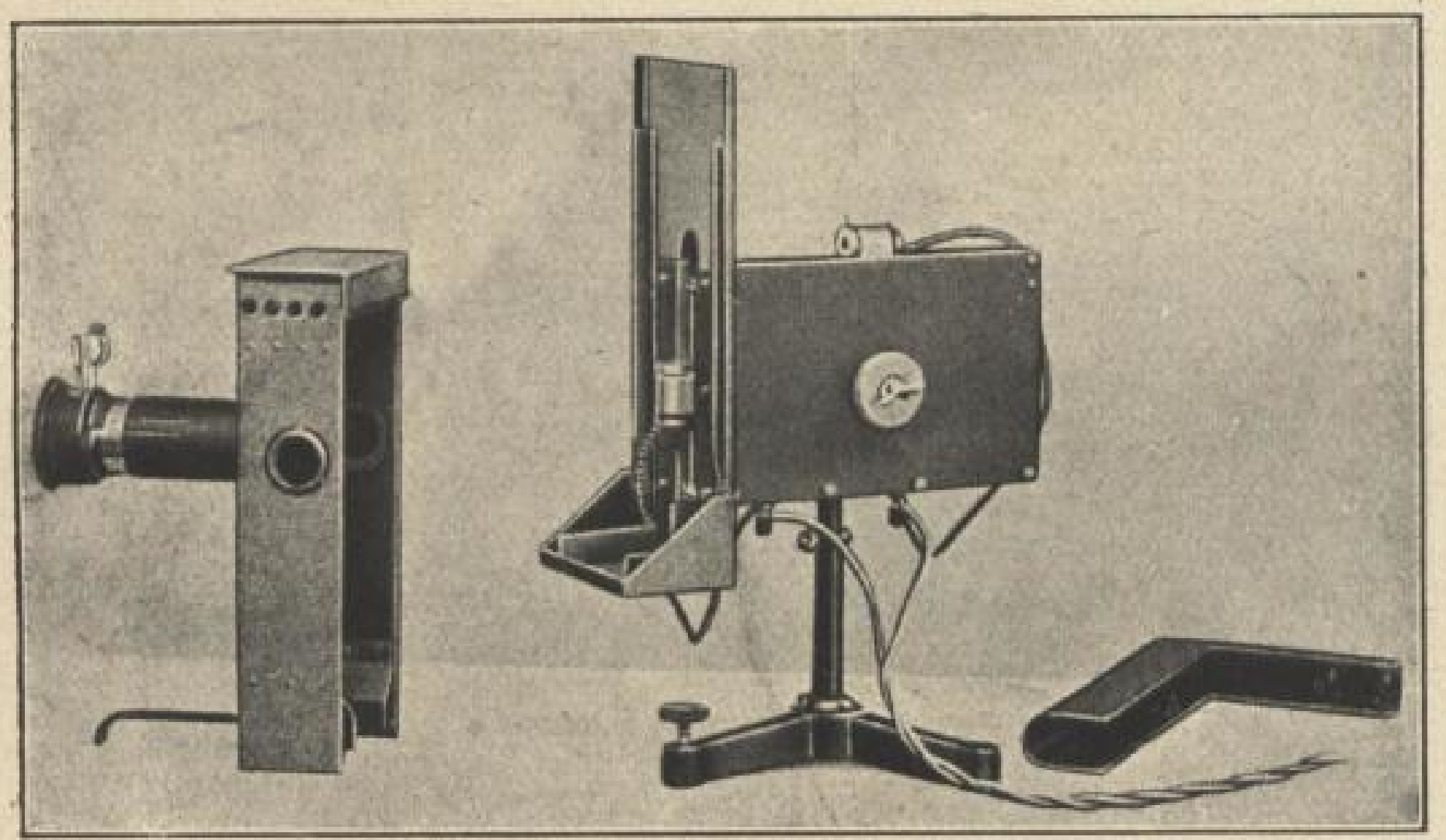

Abb. 17 . 


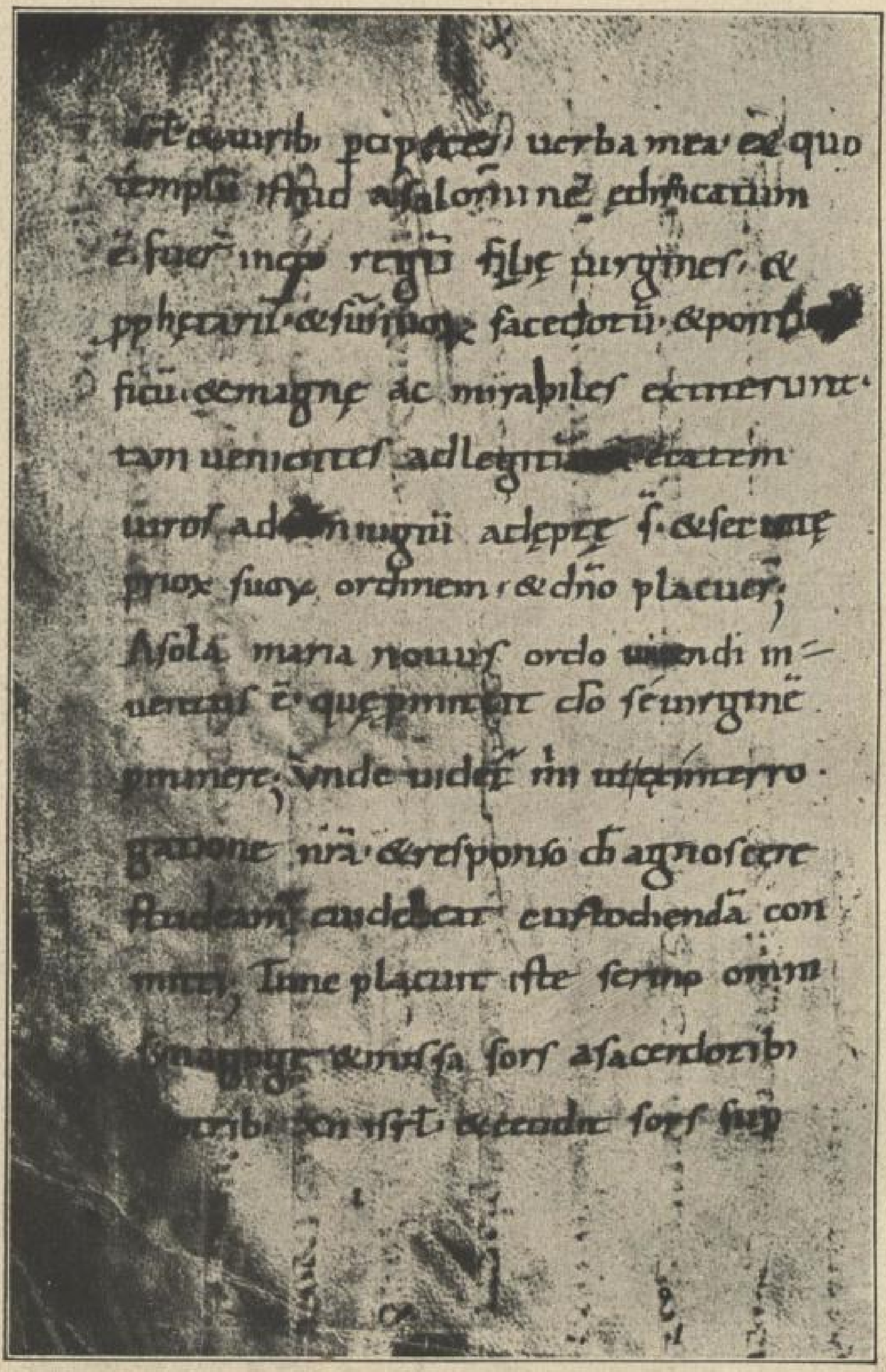

Abb. 18.

Cod. lat. mon. I9 105, p. 12v. Gewöhnliche Aufnahme. 


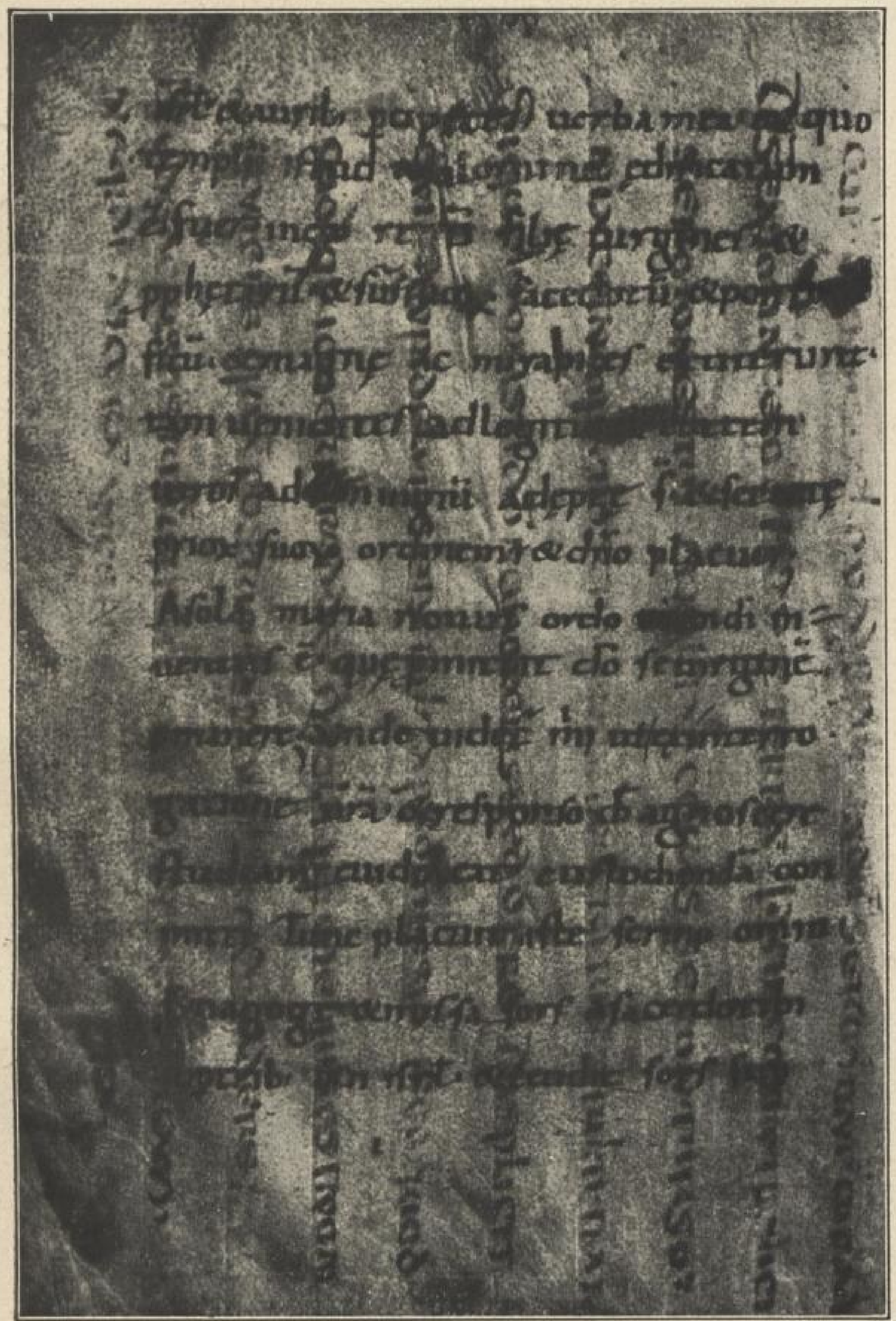

Abb. I9.

Cod. lat. mon. I9105, p. 12v.

Fluoreszenzaufnahme ohne Abdeckung der Sekundärschrift. 


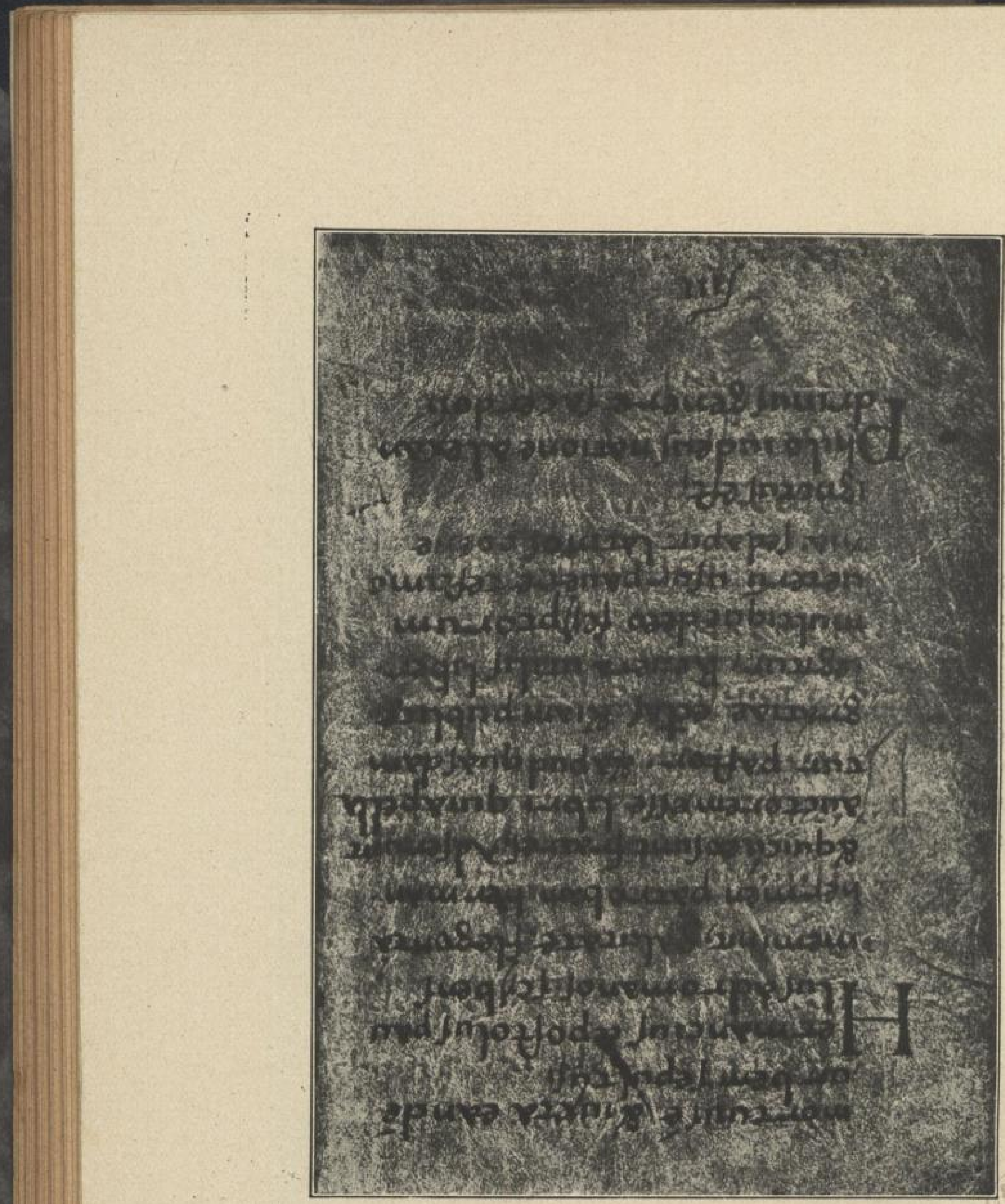

Abb. 20.

Cod. lat. mon. 6333 , p. 22 v. Aufnahme mit altem Verfahren.

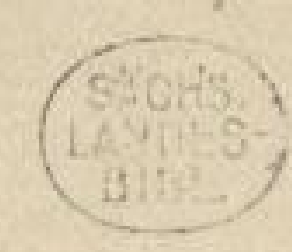




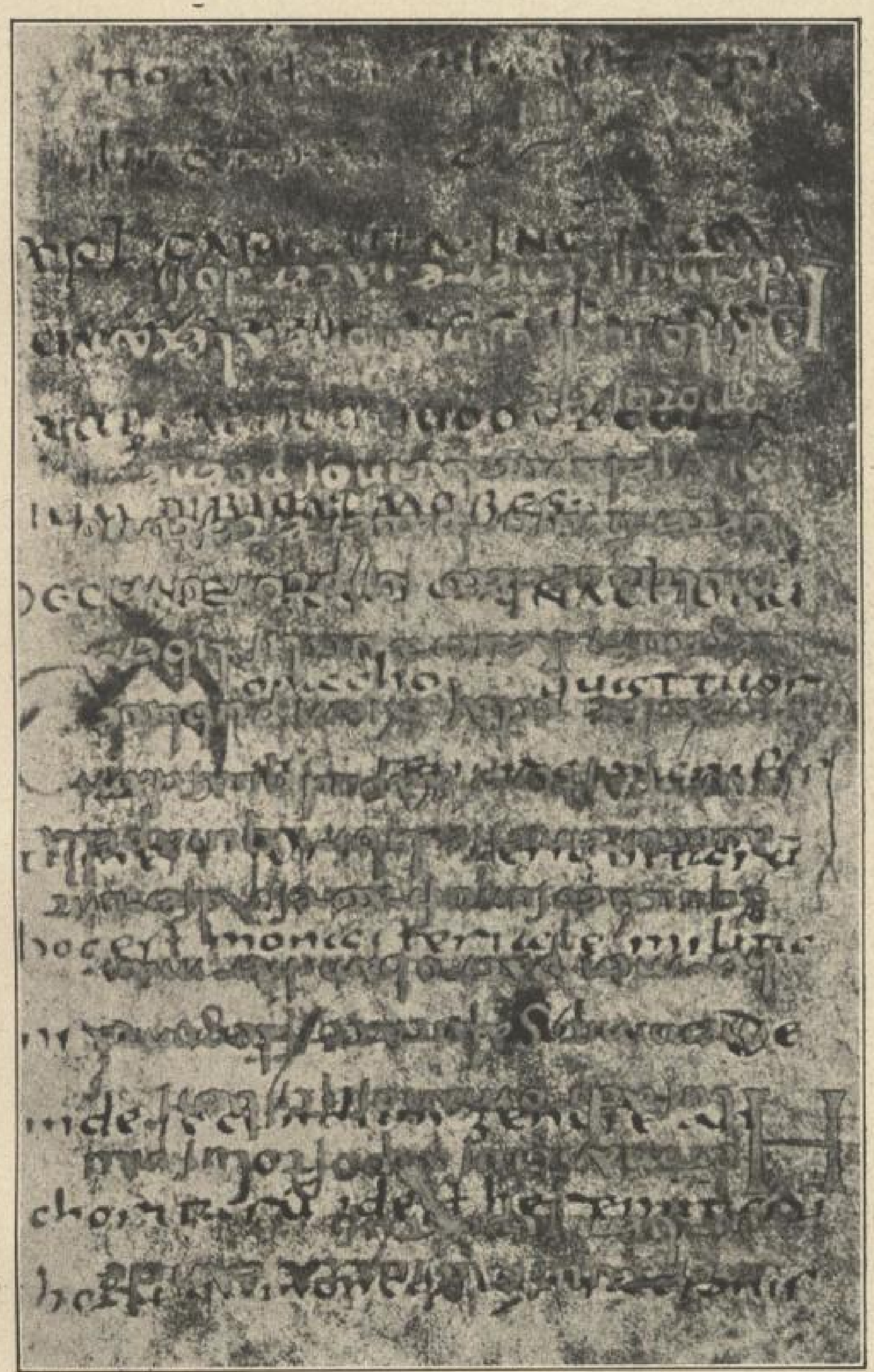

Abb. 21.

Cod. lat. mon. 6333 , p. $22^{\mathrm{v}}$.

Fluoreszenzaufnahme mit zweifacher Abdeckung der Sekundärschrift. 


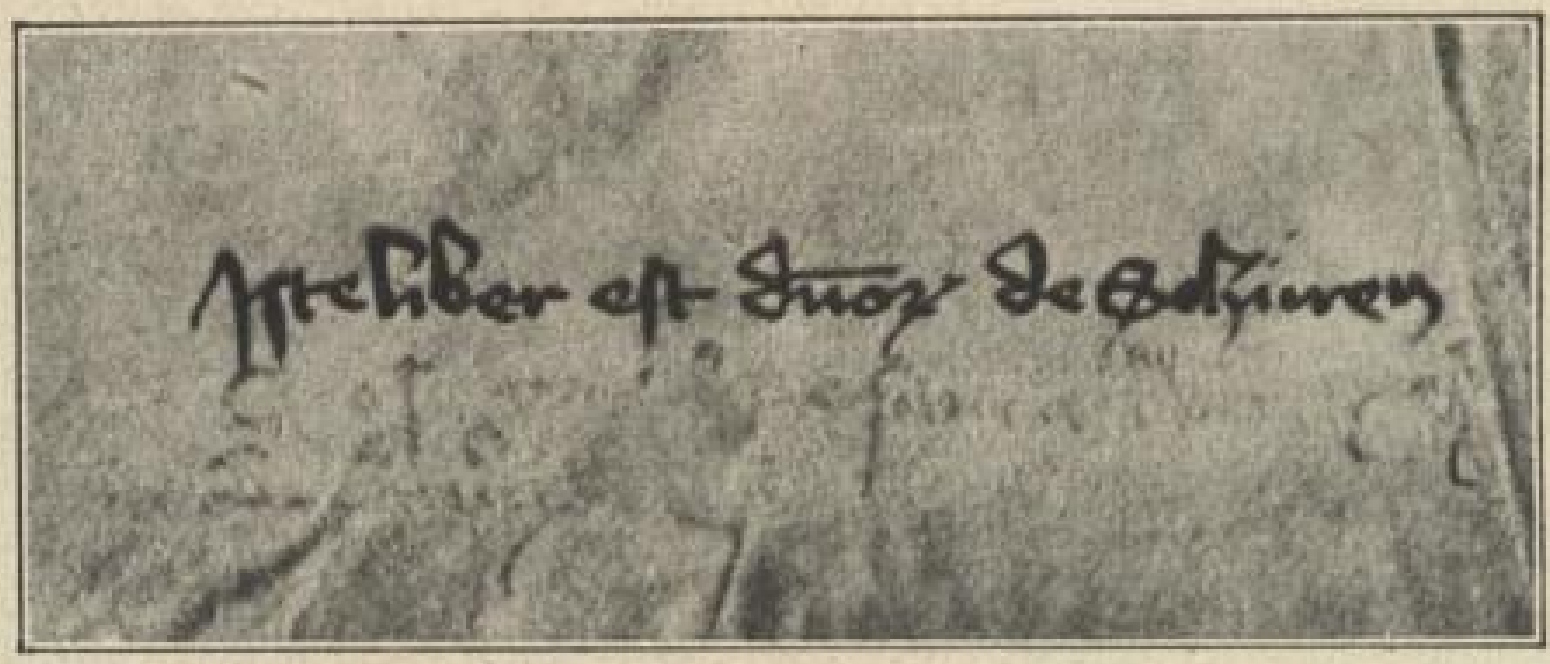

Abb. 22.

Cod. lat. mon. 17404, p. $305^{\mathrm{v}}$. Naturgetreue Aufnahme.

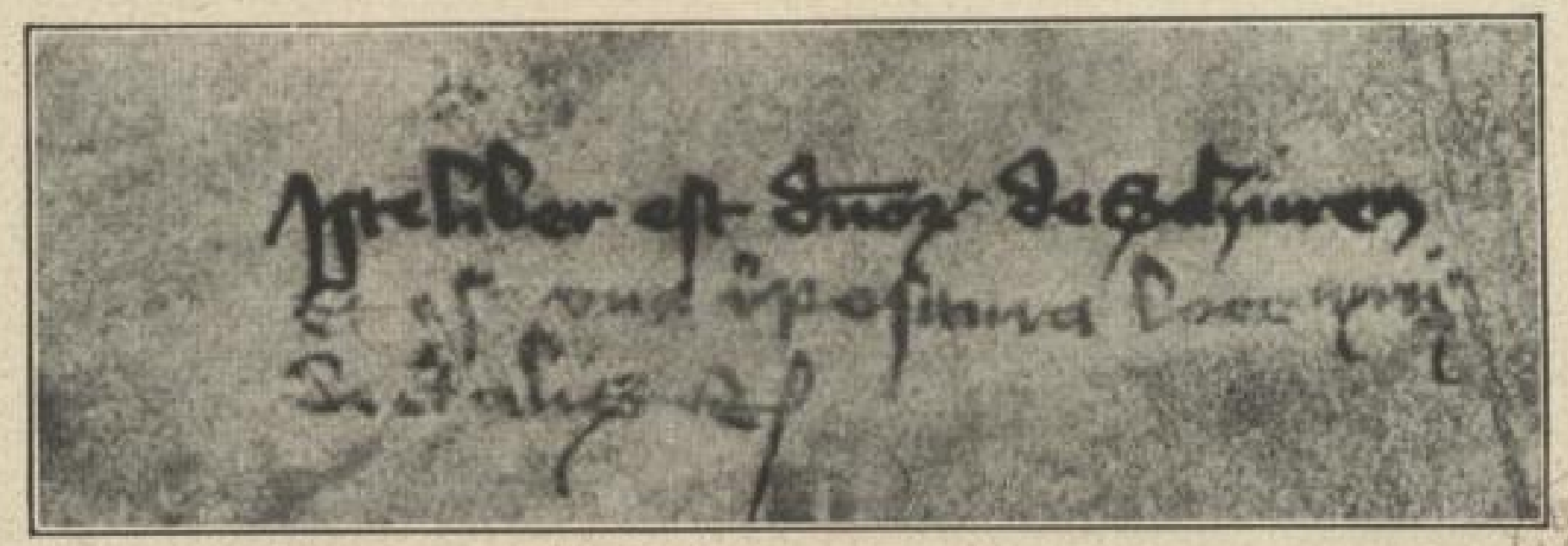

Abb. 23.

Cod. lat. mon. 17404, p. $305^{\mathrm{v}}$. Fluoreszenzaufnahme.

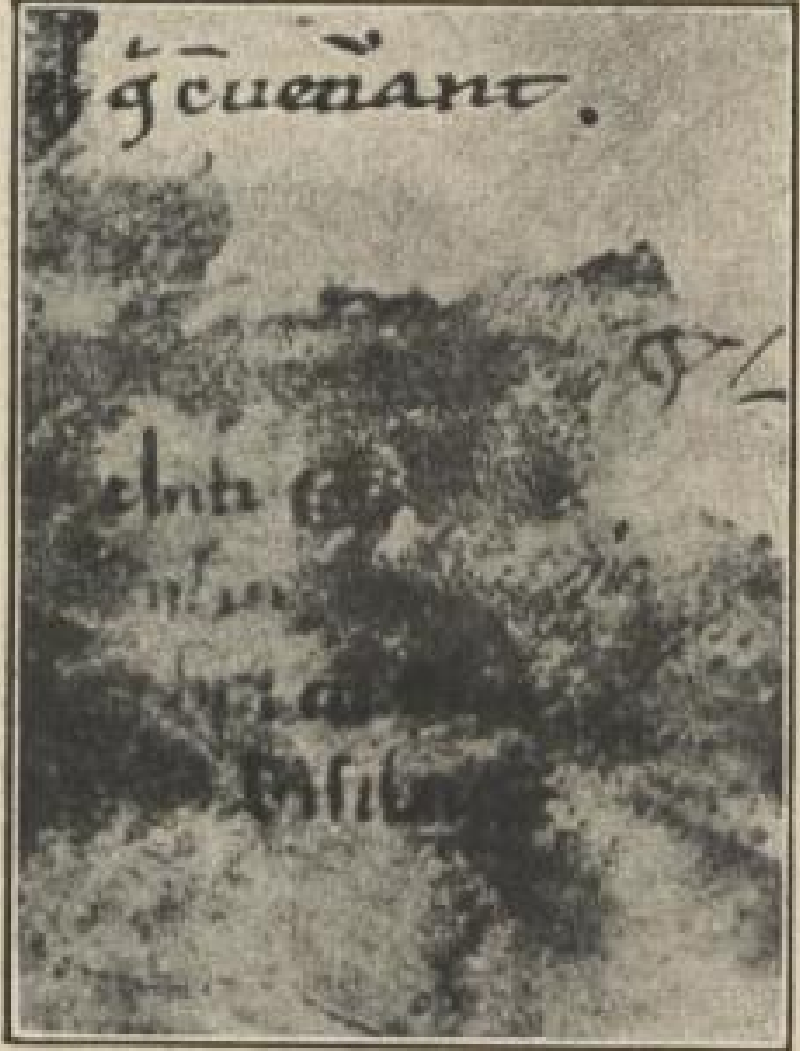

Abb. 24 .

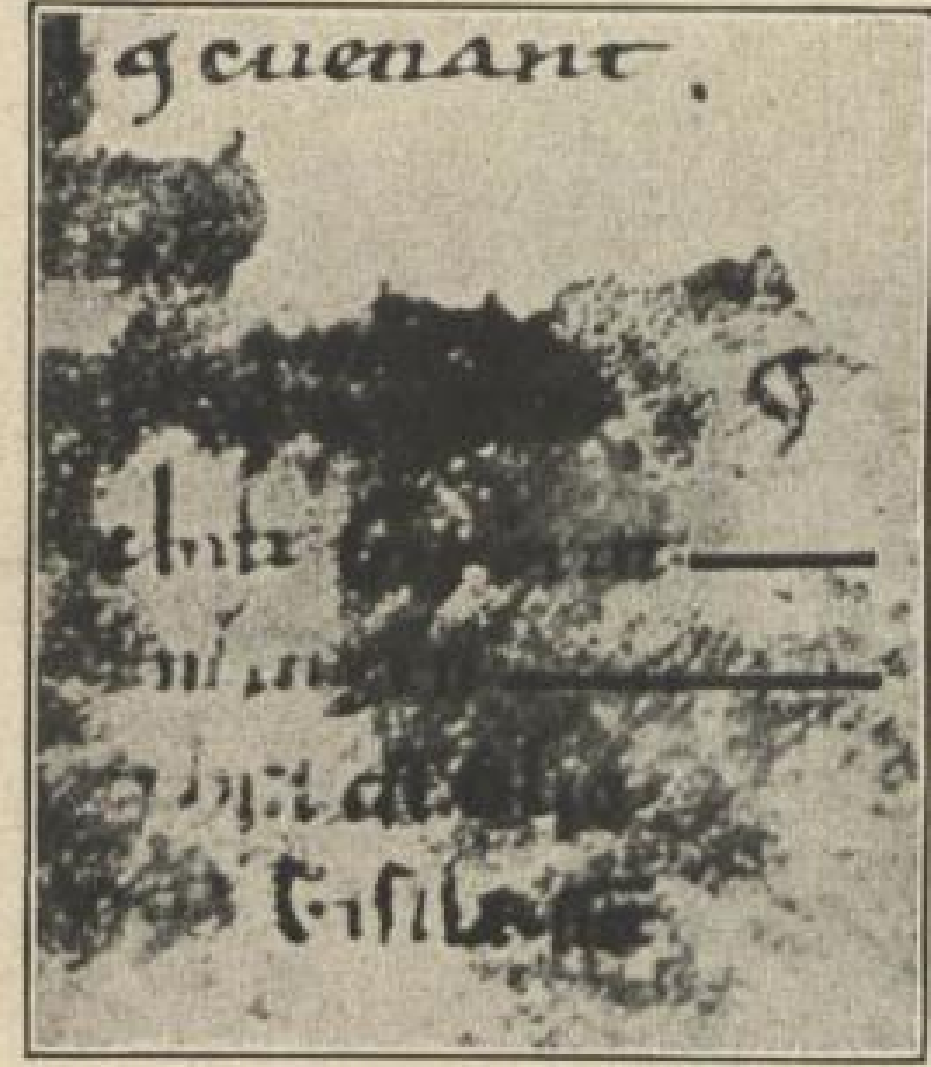

Abb. 25.

Cod. lat. mon. 1052, p. I18; $64^{\mathrm{v}}$.

Naturgetreue Aufnahme.

Fluoreszenzaufnahme. 


\section{Tarein.}

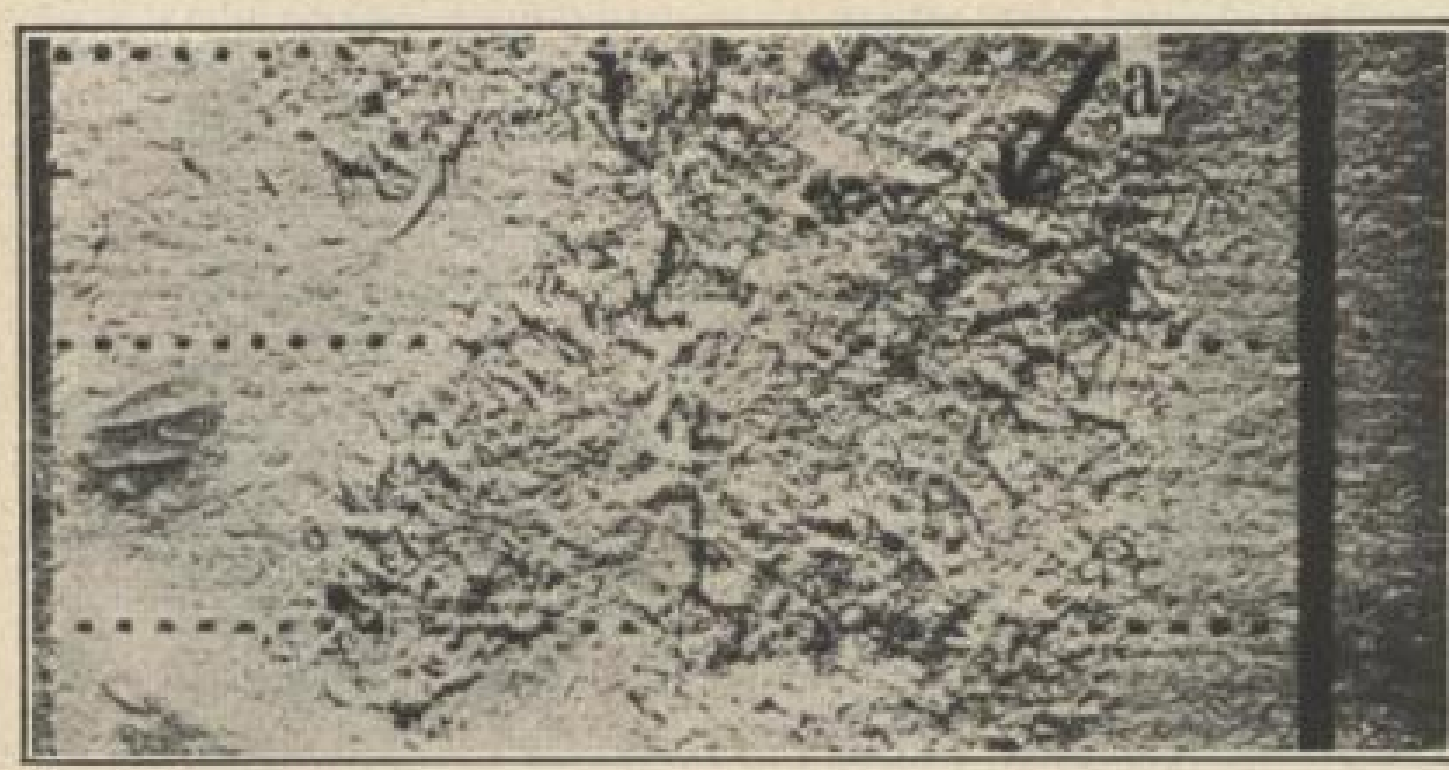

Abb. 26.

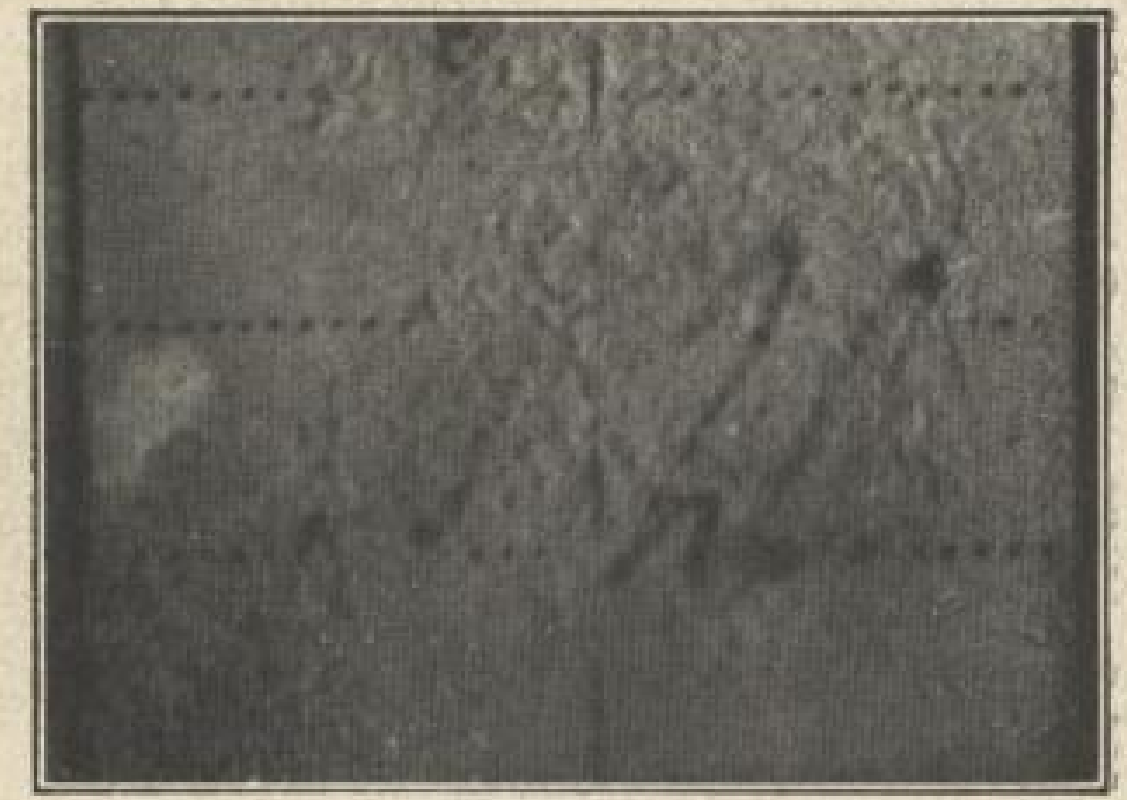

Abb. 27.

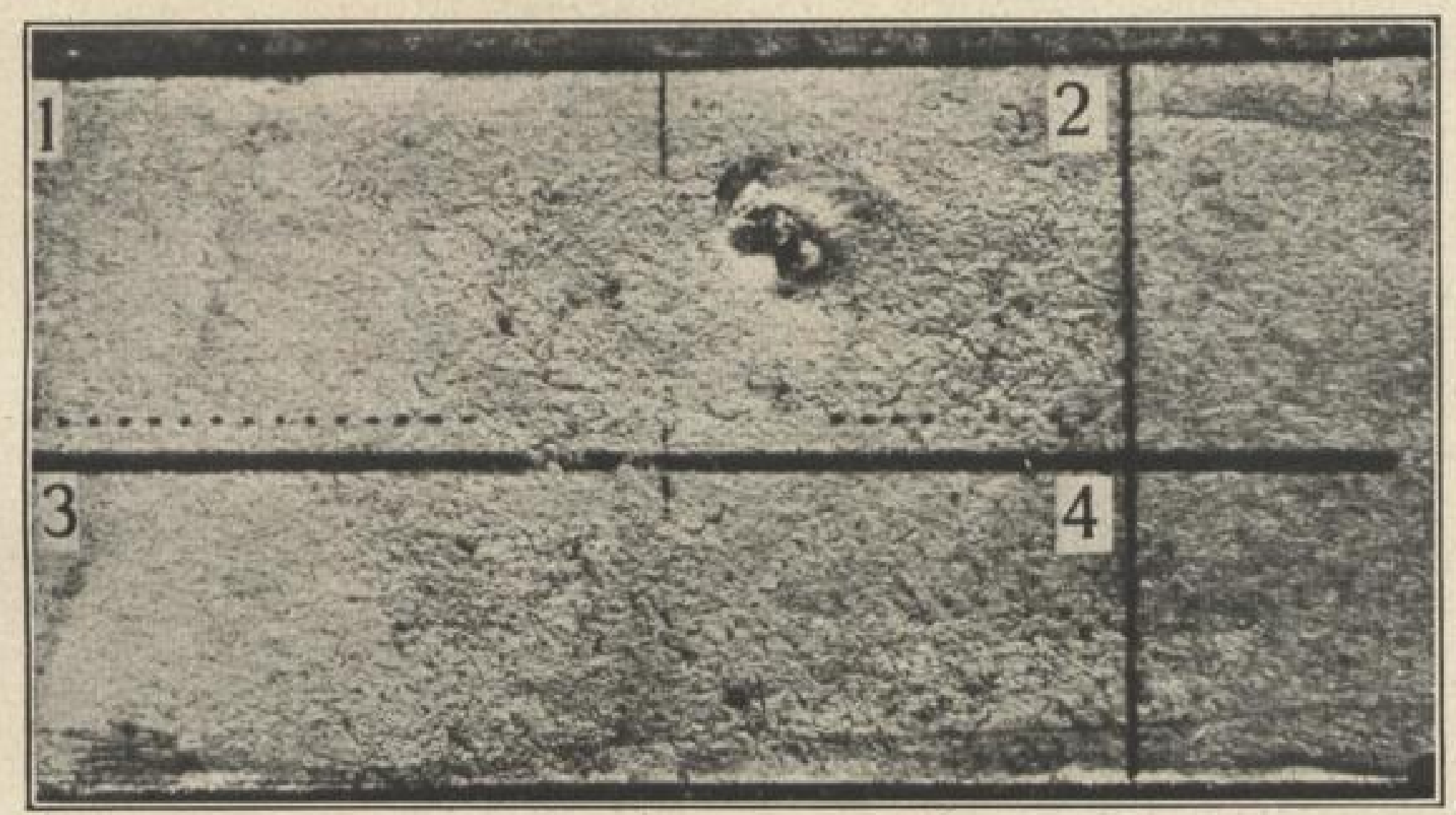

Abb. 28.

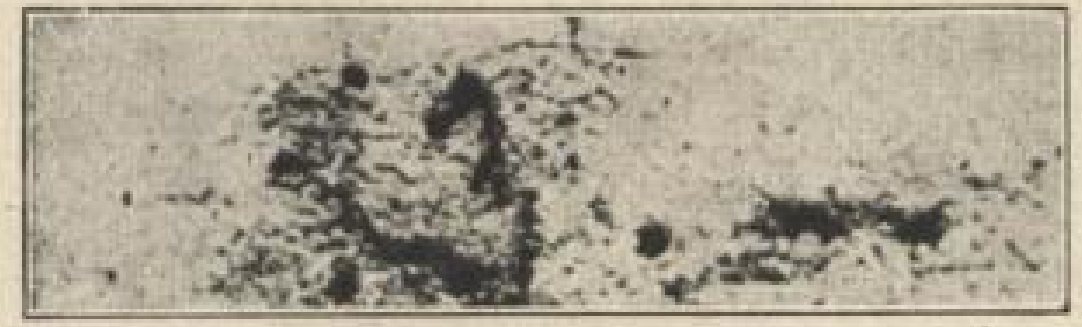

Abb. 29.

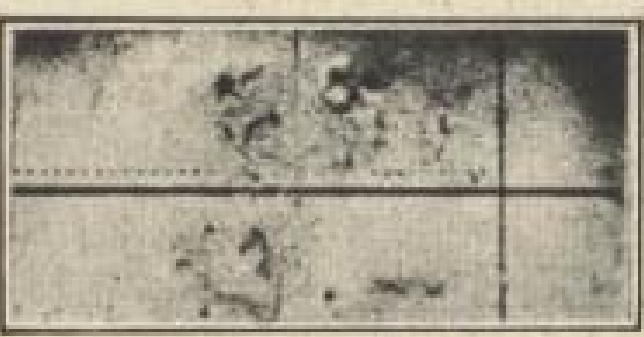

Abb. 3 .

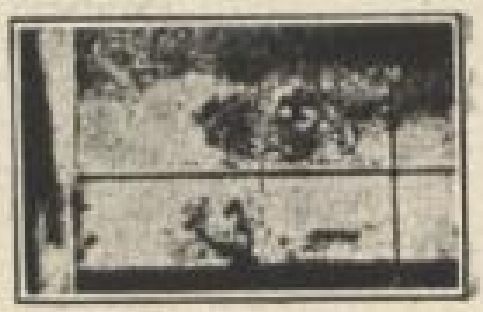

Abb. 3 r.

Erklärungen im Text oder Abschnitt ıo. 


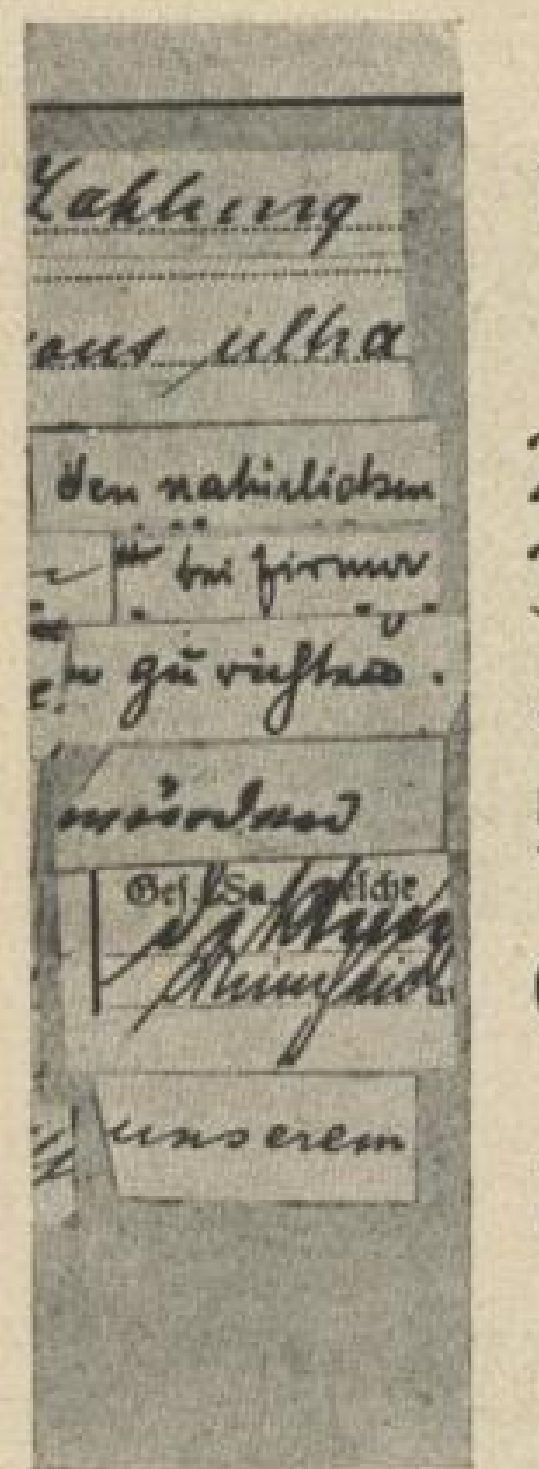

Abb. $3^{2}$.

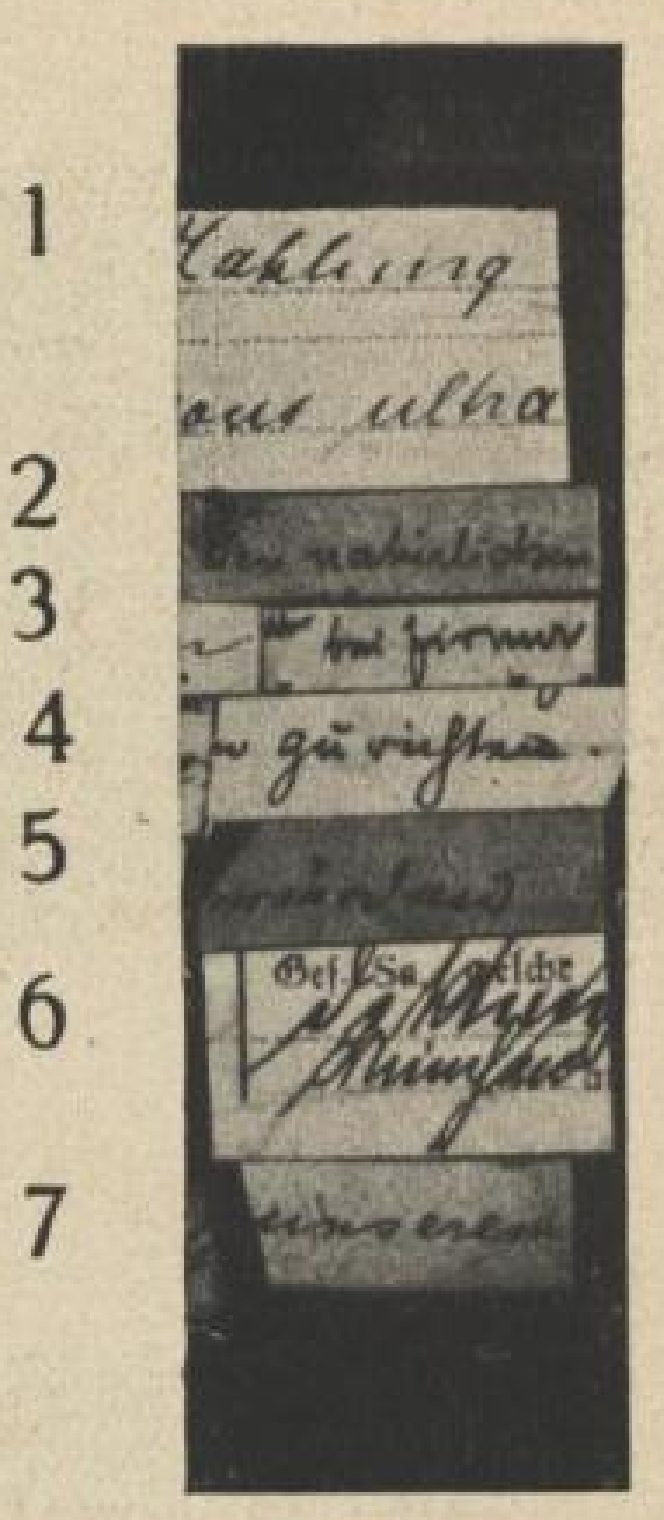

Abb. 33 .

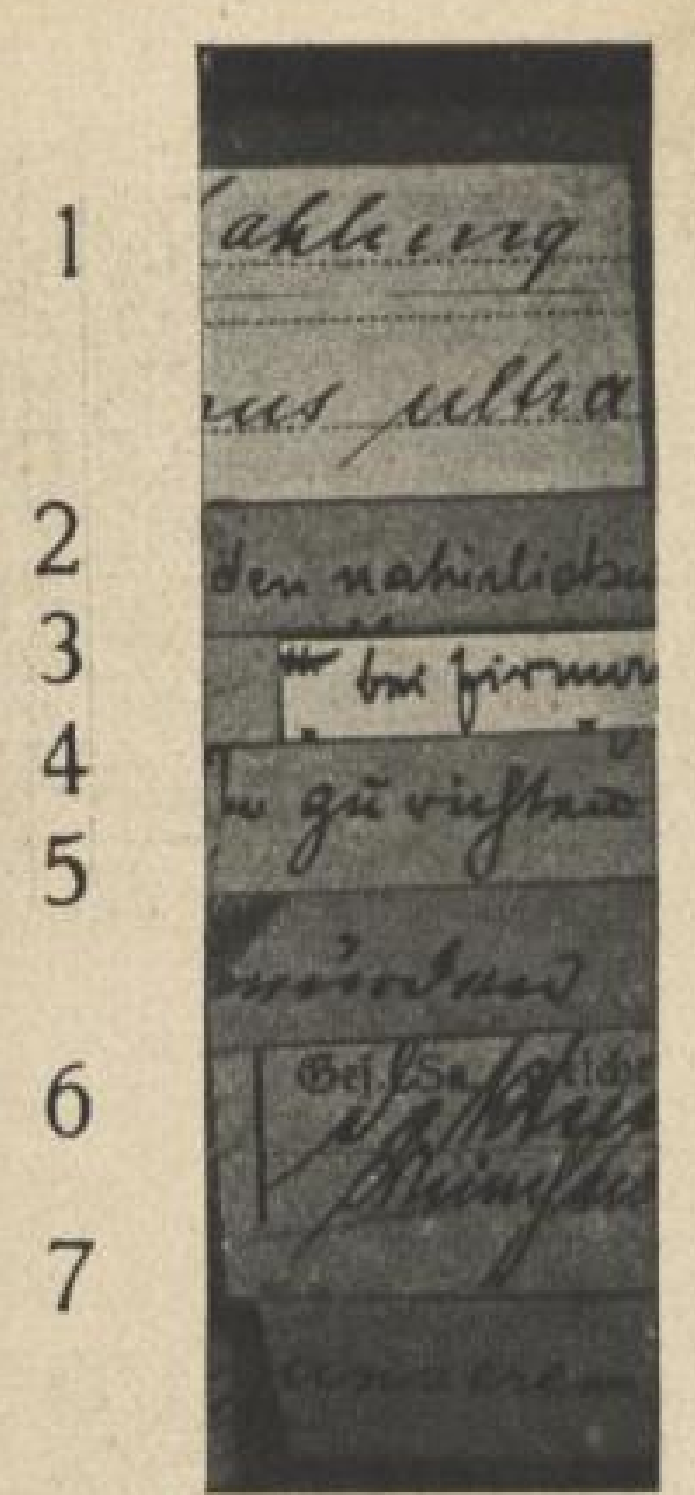

Abb. 34 .

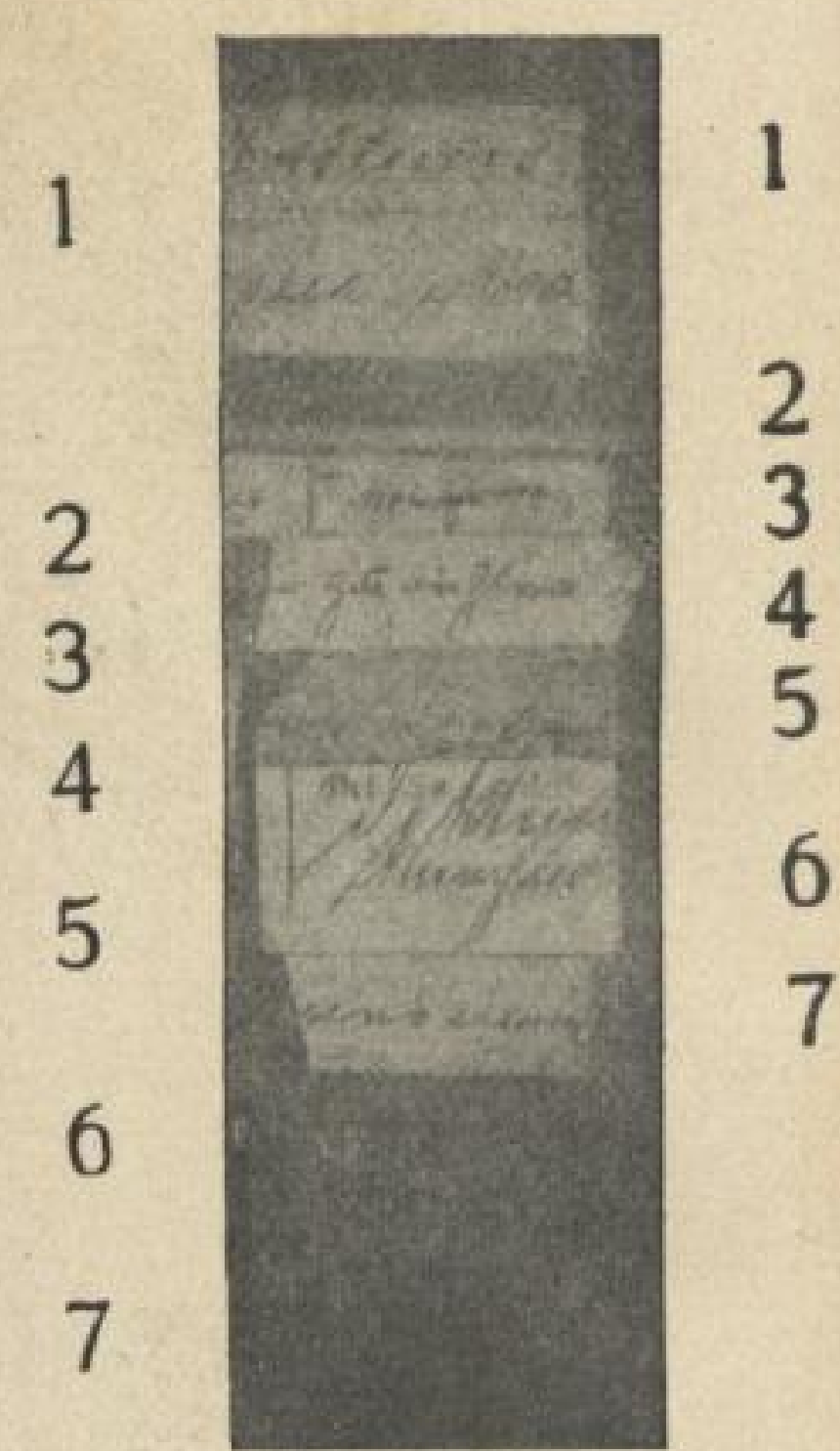

Abb. 35 .
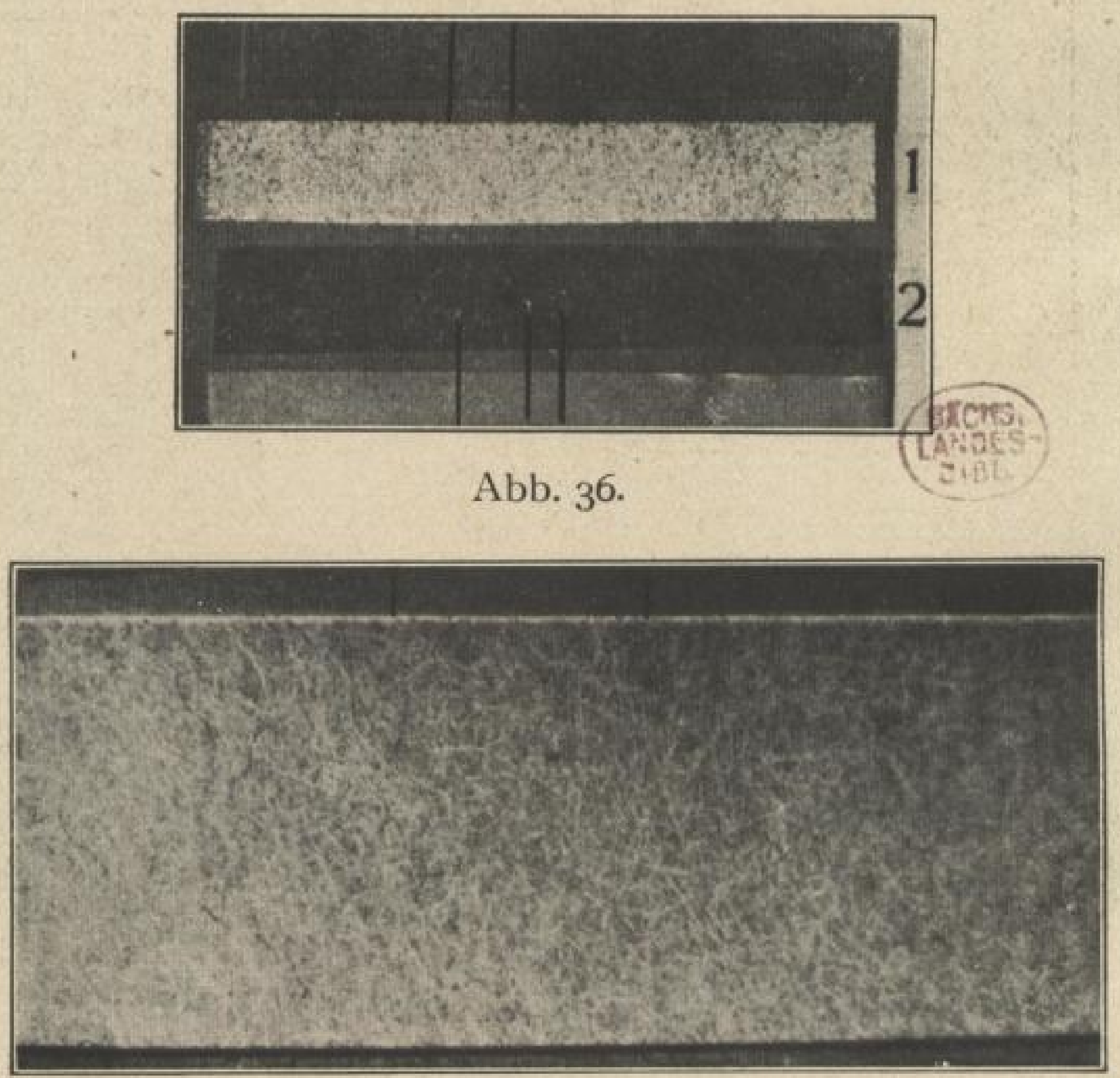

Abb. 37 .

Erklärungen im Text oder Abschnitt Io. 


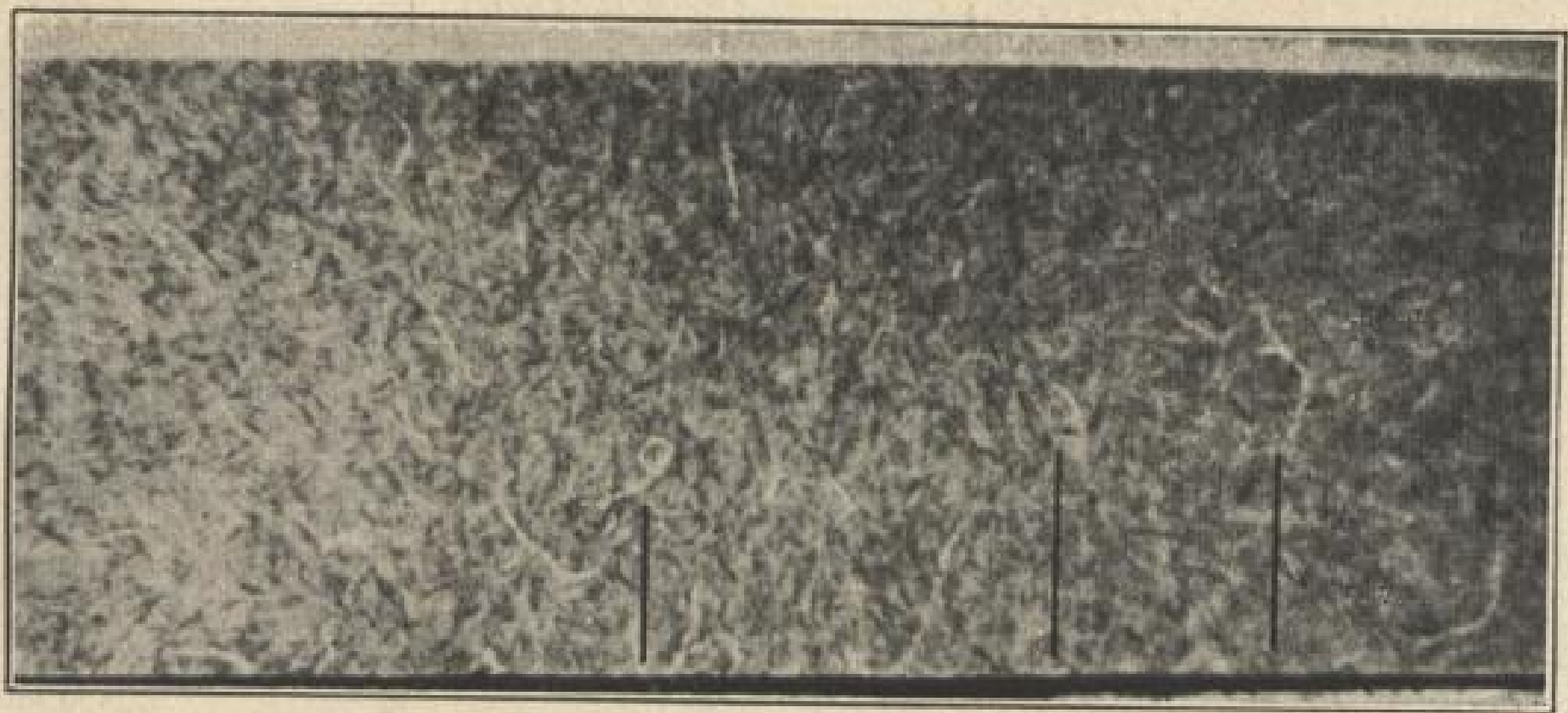

Abb. 38 .
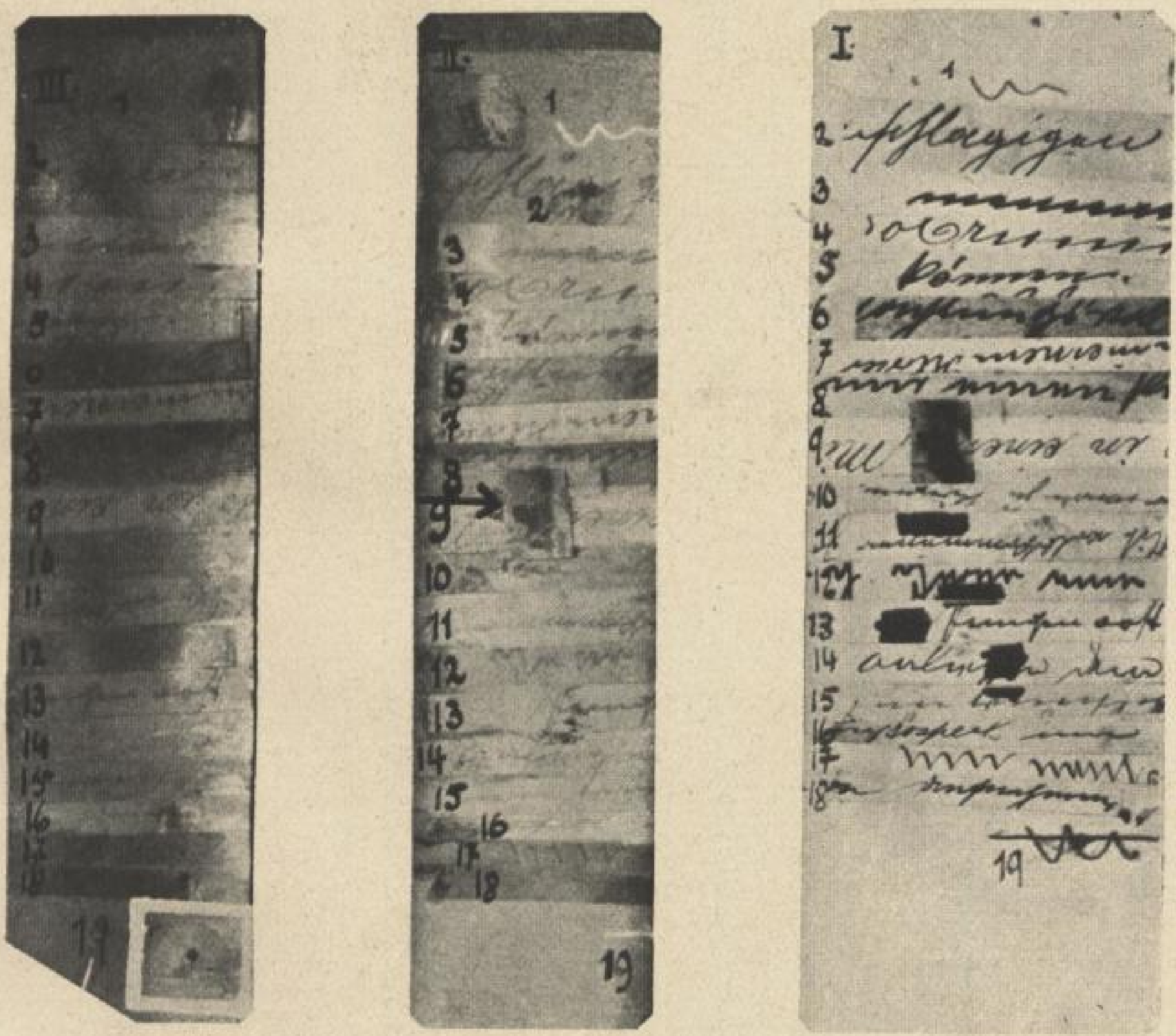

Abb. 39

Erklärungen im Text oder Abschnitt ıo. 


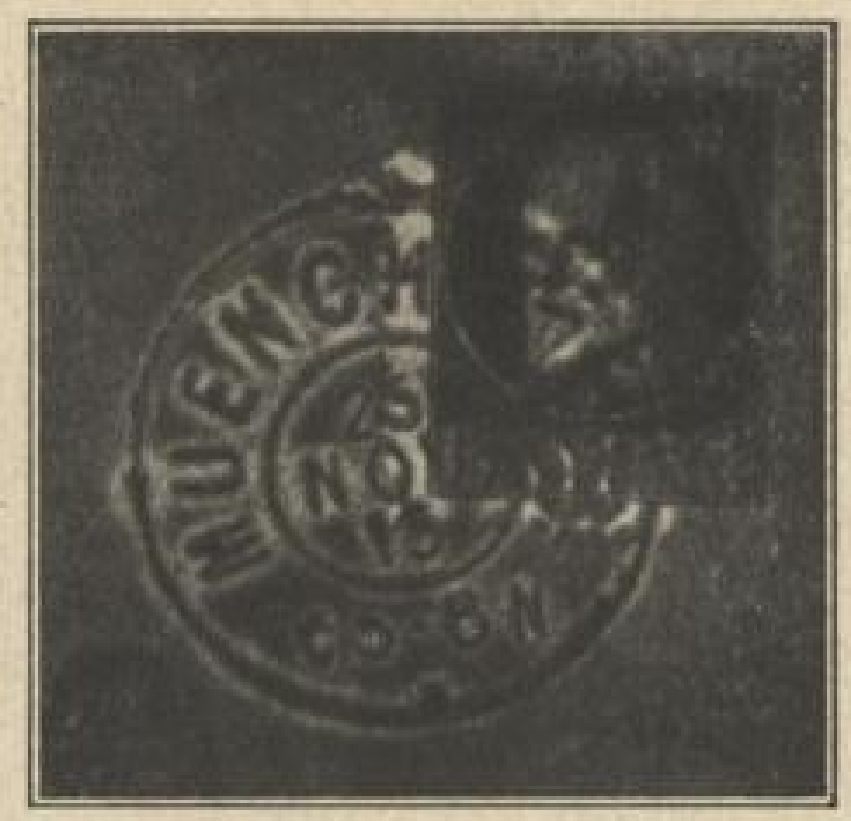

Abb. 40.

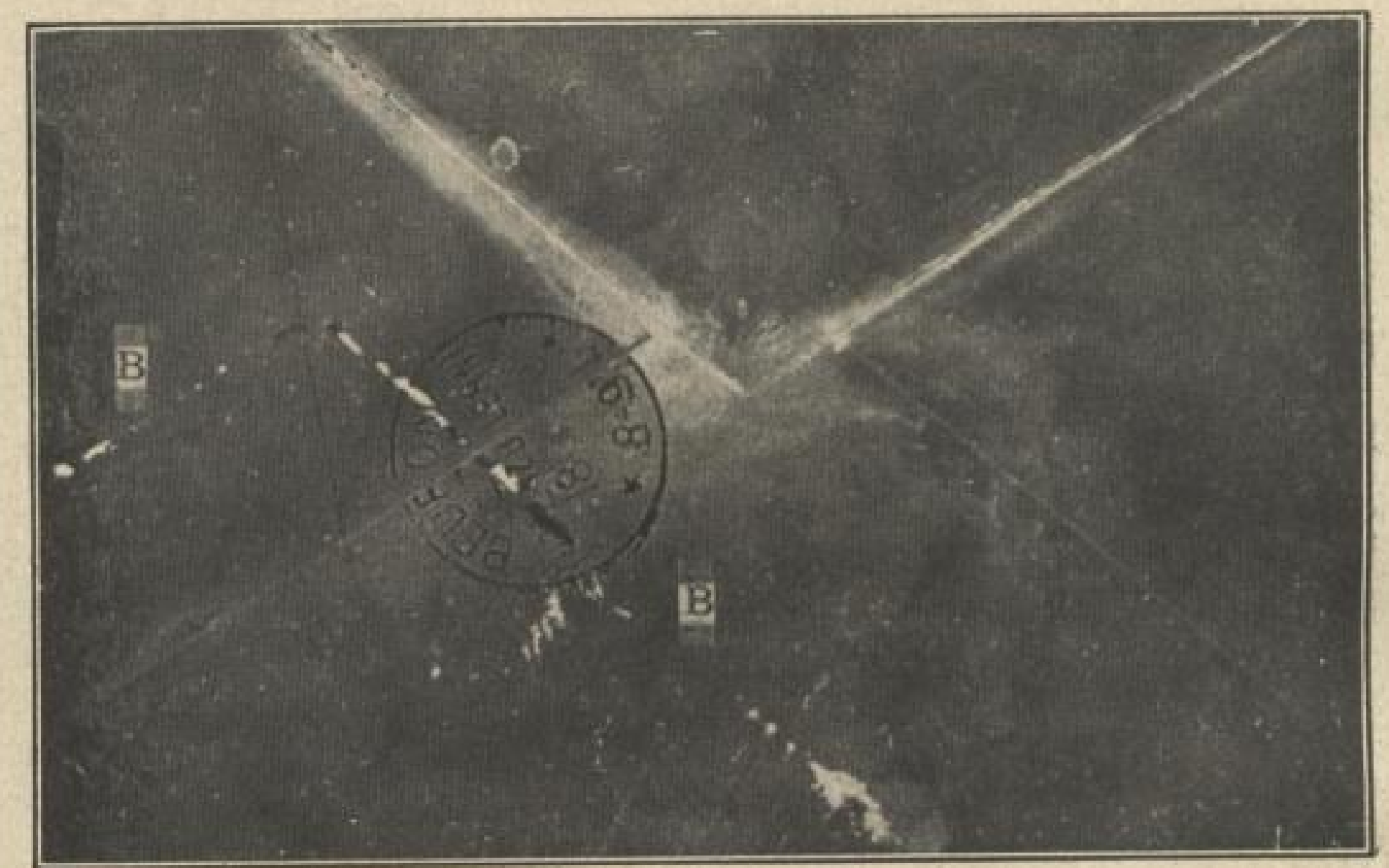

Abb. $4 \mathrm{I}$.

Abb. 41.

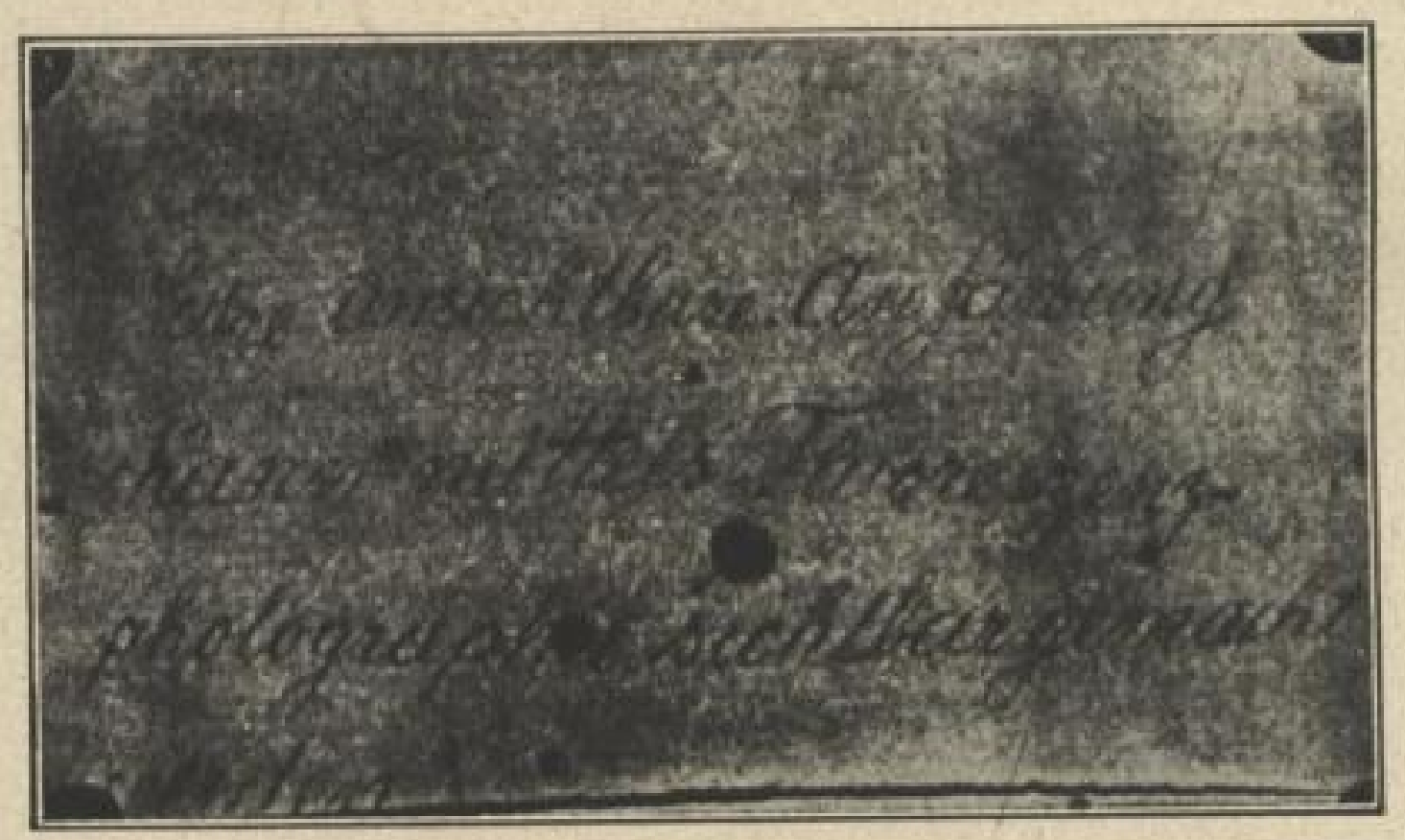

Abb. 42.

Erklärungen im Text oder Abschnitt ro.

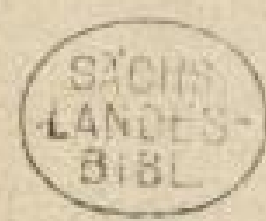




\section{Positivverfahren}

\section{nebst Diapositivverfahren und Vergröfzern.}

Vorträge über die photographischen Verfahren. Von Dozent H. Sch midt. 2. Auflage. Mit 4 Tafeln. $4,90 \mathrm{Mk}$., gebunden $7,70 \mathrm{Mk}$.

Die Mif̧erfolge in der Photographie. II. Teil: Positivverfahren. Von H. Müller. 4. Auflage.

$6,50 \mathrm{Mk}$., gebunden $8,85 \mathrm{Mk}$.

Das Arbeiten mit Gaslicht - und Bromsilberpapieren einschließlich des Postkartendrucks, sowie einer kurzen Anleitung zur Herstellung vergrößerter Bilder. Von Chemiker P. Hanneke. Mit $33 \mathrm{Ab}$ bildungen und Tafeln. $\quad 7,55 \mathrm{Mk}$., gebunden 9,60 Mk.

Das Kopieren bei elektrischem Licht. Von Dr. A. Freiherrn von Hübl. Mit 20 Abbildungen und 2 Tafeln. Neue Auflage in Vorbereitung.

Neuzeitliche photographische Kopierverfahren. Ozobromprozeß, Bromsilberpigmentpapier, Pigmentgravüre, Oeldruck, Bromöldruck, Katatypie, Druckschriften-Kopierverfahren. Von Dr. E. Stenger. 2. Auflage.

$7,05 \mathrm{Mk}$, gebunden $9,35 \mathrm{Mk}$.

Das Pigmentverfahren, der Gummi-, Oel- und Bromöldruck und verwandte photographische Kopierverfahren mit Chromsalzen. Von Hofrat Prof. Dr. J. M. Ed er. 3. Auflage. Mit 46 Abbildungen. 27,95 Mk., gebunden $33,55 \mathrm{Mk}$.

Das Bromöldruckverfahren und der Bromölumdruck. Von Dr. E. Mayer. 5. Auflage. $7,30 \mathrm{Mk}$., gebunden 9,35 Mk.

Die Selbstbereitung von Bromöldruckfarben. Von E. Guttmann. 2. Auflage.

Der Umdruck im Bromöldruckverfahren (Handpressendruck). Von E. Guttmann. 2. Auflage. 2,60 Mk.

Der Oeldruck. Von Dr. F. Fuhrmann. Mit ir Abbildungen und ${ }_{4}$ Tafeln. $5,20 \mathrm{Mk}$.

Der Gummidruck. Von Dr. W. Kösters. 2. Auflage im Druck. Mit etwa 22 Abbildungen

Neue Auflage in Vorbereitung.

Der Gummidruck und seine Verwendbarkeit als künstlerisches Ausdrucksmittel in der Photographie. Von Th. Hofmeister. 2. Auflage. Mit 4 Abbildungen und ${ }_{4}$ Tafeln.

$3,15 \mathrm{Mk}$.

Der Platindruck. Von Dr. A. Freiherrn von Hübl. 2. Auflage. Mit 7 Abbildungen.

$7,40 \mathrm{Mk}$.

Die Ozotypie. Ein Verfahren zur Herstellung von Pigmentkopien ohne Uebertragung. Von Dr. A. Freiherrn von Hübl. 3,50 Mk.

Die Diapositivverfahren. Praktische Anleitung zur Herstellung von Fenster-, Stereoskop- und Projektionsbildern usw. Von G. Mer cator. 3. Auflage. $4,70 \mathrm{Mk}$, gebunden $6,75 \mathrm{Mk}$.

Handbuch des Vergröfgerns auf Papieren und Platten. Von Prof. Dr. F. Stolze. 4. Auflage im Druck. Neu bearbeitet von P. Thieme.

\section{Retusche und Kolorieren.}

Die photographische Retusche mit besonderer Berücksichtigung der modernen chemischen, mechanischen und optischen Hilfsmittel. Nebst einer Anleitung zum Kolorieren von Photographien. Von G. Mercator. 5. Auflage. $4,70 \mathrm{Mk}$., gebunden $6,65 \mathrm{Mk}$.

Anleitung zum Kolorieren photographischer Bilder jeder Art mittels Aquarell-, Lasur-, Oel-, Pastell-und anderen Farben. Von G. Mer cator. 2. Auflage.

$5,20 \mathrm{Mk}$. 


\section{Apparate nebst Zubehör.}

Das Arbeiten mit kleinen Kameras nebst praktischer Anleitung zu der Entwicklung der kleinen Negative, sowie der Herstellung von Kopien und Bildvergrößerungen. Von Chemiker P. Hanneke. 3. Auflage. Mit 64 Abbildungen. $4,70 \mathrm{Mk}$., gebunden $6,6_{5} \mathrm{Mk}$.

Die Spiegelreflexkamera. Von A. Mayer, neu bearbeitet von P. Hanneke. 2. Auflage im Druck. Mit 52 Abbildungen.

4,20 Mk., gebunden 6,- Mk.

Die Stereoskopie und das Stereoskop in Theorie und Praxis. Von Prof. Dr. F. Stolze. 2. Auflage. Mit 46 Abbildungen.

9,75 Mk., gebunden $12,35 \mathrm{Mk}$.

Die Panoramenapparate. Von Prof. Dr. F. Stolze. Mit 33 Abb. 6,- Mk.

Die photographischen Lichtfilter. Von Dr. A. Freiherrn von Hübl. Mit I8 Abbildungen und 5 Tafeln. $8,60 \mathrm{Mk}$.

Die Belichtungsmesser der photographischen Praxis. Von Dr. R. H. Blochmann. Mit 6 Abbildungen. $3,80 \mathrm{Mk}$., gebunden $5,75 \mathrm{Mk}$.

\section{Photographieren bei künstlichem Licht.}

Das Photographieren mit Blitzlicht. Von Dozent H. Schmidt. 2. Auflage im Druck. Mit 1 Tafel und 60 Abbildungen.

$8,20 \mathrm{Mk}$., gebunden Io. - Mk.

Die Photographie bei künstlichem Licht, Spektrumphotographie, Aktinometrie und die chemischen Wirkungen des farbigen Lichtes. Von Hofrat Prof. Dr. J. M. Eder. 3. Auflage. Mit 409 Abbildungen und Io Tafeln. $46,55 \mathrm{Mk}$., gebunden 52,05 Mk.

\section{Farbenphotographie.}

Die Theorie und Praxis der Farbenphotographie mit Autochrom und anderen Rasterfarbenplatten. Von Dr. A. Freiherrn von Hübl. 4. Auflage. Mit 8 Abbildungen.

$4,70 \mathrm{Mk}$., gebunden $6,65 \mathrm{Mk}$.

Die Photographie in natürlichen Farben mit besonderer Berücksichtigung des Lippmannschen Verfahrens, sowie jener Methoden, welche bei einmaliger Belichtung ein Bild in Farben liefern. Von Prof. Dr. E. Valenta. 2. Auflage. Mit 32 Abbildungen und 6 Tafeln.

II,70 Mk., gebunden $\mathrm{I}_{4}, 05 \mathrm{Mk}$.

Die Dreifarbenphotographie mit besonderer Berücksichtigung des Dreifarbendruckes und ähnlicher Verfahren. Von Dr. A. Freiherrn von Hübl. 3. Auflage. Mit 40 Abbildungen und 4 Tafeln. I3,80 Mk., gebunden $16,40 \mathrm{Mk}$.

\section{Kalender und Jahrbücher.}

Photographischer Notizkalender. Begründet von Geh. Reg.-Rat Prof. Dr. A. Miethe und Prof. Dr. F. Stolze, neu bearbeitet von Chemiker P. Hanneke und Schriftleiter W. König. Erscheint bereits seit 1896 regelmäßig zur Jahreswende. Inhalt: Kalendarium, Rezepte, Tabellen. Ratschläge, praktische Winke, rechtliche und gewerbliche Fragen. Taschengröße,

Gebunden 5,20 Mk.

Jahrbuch für Photographie und Reproduktionstechnik. Von Hofrat Prof. Dr. J. M. Ed er. Berichte über die alljährlichen Fortschritte der Wissenschaft und Praxis auf jenen Gebieten. Erscheint bereits seit 1887 . Preis eines Jahrganges 17,55 Mk., gebunden 21,20 Mk. Die photographische Kunst. Siehe unter "Künstlerische Photographie". 
Tarein.

Palimpseste ("Thotographic)

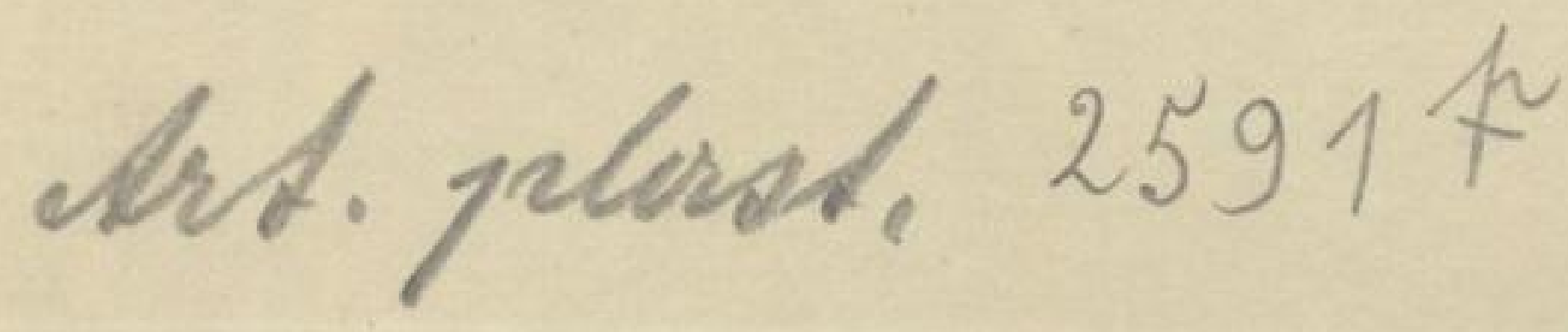

SLUB Wir fohren Wissen. gefördert von der
Deutschen Forschungsgemeinschaft 


\section{SLUB DRESDEN

